\title{
REVIEW
}

Open Access

\section{Evidence on physical activity and osteoporosis prevention for people aged 65+ years: a systematic review to inform the WHO guidelines on physical activity and sedentary behaviour}

Marina B. Pinheiro ${ }^{1,2^{*}}$ (D) Juliana Oliveira ${ }^{1,2}$, Adrian Bauman ${ }^{3}$, Nicola Fairhall ${ }^{1,2}$, Wing Kwok Kw $^{1,2}$ and Catherine Sherrington ${ }^{1,2}$

\begin{abstract}
Background: Various physical activity interventions for prevention and treatment of osteoporosis have been designed and evaluated, but the effect of such interventions on the prevention of osteoporosis in older people is unclear. The aim of this review was to investigate the association between physical activity and osteoporosis prevention in people aged 65 years and above.
\end{abstract}

Methods: A systematic review was conducted and searches for individual studies were conducted in PubMed (January 2010 to March 2020) and for systematic reviews were conducted in PubMed, Embase, CINAHL and SPORTDiscus (January 2008 to July 2020). Records were screened according to the following eligibility criteria: i) population: adults aged 65 years and older; ii) exposure: greater volume, duration, frequency, or intensity of physical activity; iii) comparison: no physical activity or lesser volume, duration, frequency, or intensity of physical activity; iv) outcome: osteoporosis related measures (e.g., bone mineral density). The methodological quality of included studies was assessed and meta-analysis summarised study effects. The GRADE approach was used to rate certainty of evidence.

\footnotetext{
* Correspondence: Marina.pinheiro@sydney.edu.au

'Institute for Musculoskeletal Health, The University of Sydney and Sydney Local Health District, Sydney, Australia

${ }^{2}$ School of Public Health, Faculty of Medicine and Health, The University of Sydney, Sydney, Australia

Full list of author information is available at the end of the article
}

C C The Author(s). 2020 Open Access This article is licensed under a Creative Commons Attribution 4.0 International License, which permits use, sharing, adaptation, distribution and reproduction in any medium or format, as long as you give appropriate credit to the original author(s) and the source, provide a link to the Creative Commons licence, and indicate if changes were made. The images or other third party material in this article are included in the article's Creative Commons licence, unless indicated otherwise in a credit line to the material. If material is not included in the article's Creative Commons licence and your intended use is not permitted by statutory regulation or exceeds the permitted use, you will need to obtain permission directly from the copyright holder. To view a copy of this licence, visit http://creativecommons.org/licenses/by/4.0/. The Creative Commons Public Domain Dedication waiver (http://creativecommons.org/publicdomain/zero/1.0/) applies to the data made available in this article, unless otherwise stated in a credit line to the data. 
(Continued from previous page)

Results: We included a total of 59 studies, including 12 observational studies and 47 trials. Within the included trials, 40 compared physical activity with no intervention controls, 11 compared two physical activity programs, and six investigated different doses of physical activity. Included studies suggest that physical activity interventions probably improve bone health among older adults and thus prevent osteoporosis (standardised effect size $0.15,95 \% \mathrm{Cl} 0.05$ to $0.25,20$ trials, moderate-certainty evidence, main or most relevant outcome selected for each of the included studies). Physical activity interventions probably improve lumbar spine bone mineral density (standardised effect size 0.17, 95\% Cl 0.04 to $0.30,11$ trials, moderate-certainty evidence) and may improve hip (femoral neck) bone mineral density (standardised effect size 0.09, $95 \% \mathrm{Cl}-0.03$ to $0.21,14$ trials, low-certainty evidence). Higher doses of physical activity and programs involving multiple exercise types or resistance exercise appear to be most effective. Typical programs for which significant intervention impacts were detected in trials were undertaken for 60+ mins, 2-3 times/week for 7+ months. Observational studies suggested a positive association between long-term total and planned physical activity on bone health.

Conclusions: Physical activity probably plays a role in the prevention of osteoporosis. The level of evidence is higher for effects of physical activity on lumbar spine bone mineral density than for hip. Higher dose programs and those involving multiple exercises and resistance exercises appear to be more effective.

Keywords: Physical activity, Osteoporosis, Bone mineral density, Older people, Review

\section{Background}

Osteoporosis is a major public health problem and is characterised by micro-architectural deterioration of bone tissue and low bone mineral density (BMD) which leads to reduced bone strength, increased bone fragility and a consequent increase in risk of skeletal fractures $[1,2]$. Osteoporosis is known as a 'silent disease' as it is frequently undiagnosed until a symptomatic fracture occurs - usually at an older age [3]. The most common clinical manifestations of osteoporosis are fractures of the hip, vertebrae or wrist, with incidence increasing with age. Osteoporotic fractures are responsible for excess morbidity, mortality, reduction in quality of life, institutionalization and economic costs [1, 4-7]. For instance, in the UK it is estimated that fragility fractures cost the NHS $£ 4.4$ billion per year [8] and in the USA osteoporosis cost US\$57 billion in 2018 with this figure projected to grow to over US\$95 billion yearly by 2040 [9]. In light of worldwide increases in life expectation as well as the burden placed by osteoporosis fractures on societies, health systems and individuals, effective osteoporosis prevention strategies are essential.

Low bone mass is recognised as an important risk factor for fracture and therefore, a key target for osteoporosis prevention [1]. It is thought that disuse and inactivity generates unloading of the skeletal system resulting in reduced bone mass. Conversely, physical activity is thought to stimulate bone growth and preserve bone mass. Physical activity is an umbrella term that includes leisure time physical activity (exercise, sport), activities of daily living, household tasks and work [10]. The benefits of physical activity for healthy ageing are well established $[11,12]$ and various physical activity interventions for prevention and treatment of osteoporosis have been designed and evaluated. Various guidelines recommend engagement in physical activity for the management of osteoporosis [1], including for older people [13], however the effects of such interventions in older people who have not been diagnosed with osteoporosis i.e., in osteoporosis prevention have not been summarised. A summary of the evidence in this field is crucially important to enable specific recommendations on physical activity engagement for osteoporosis prevention to be made.

Previous reviews investigating the association between physical activity and osteoporosis prevention have only focused on specific types of physical activity, such as exercise [14-18], walking [19, 20], or sport [21], single body parts [22], male [15, 23] or female [14, 17, 20], and most were not specifically focused on older people or prevention. To address this evidence gap, and provide a comprehensive summary of the evidence in the field, we conducted a review investigating the effect of physical activity for prevention of osteoporosis in older people [24]. Given the worldwide low levels of physical activity, particularly pronounced in older people [25], a summary of the evidence on the effects of physical activity on the prevention of osteoporosis is important to inform public health initiatives and planning.

This review aimed to investigate the association between physical activity and osteoporosis prevention in older people (aged 65 years and above). The questions were: i) What is the association between physical activity and osteoporosis prevention in older people ( $>64$ years old)? ii) Is there a dose response association (volume, duration, frequency, intensity) between physical activity and prevention of osteoporosis? iii) Does the association vary by type or domain of physical activity? The focus was on primary prevention studies i.e., studies in the general community rather than studies in those with existing osteoporosis. 


\section{Methods}

We conducted a systematic review investigating the association between physical activity and osteoporosis prevention in older people. This review was commissioned by the World Health Organization (WHO) to assist the Guideline Development Group (GDG) develop the guidelines on physical activity and sedentary behaviour (2020) $[26,27]$. It was submitted to the GDG for their consideration as they formulated their recommendations. The GDG decided on the scope of the guideline, the PICO (Population, Intervention, Comparison, Outcome) question, and the search strategy. The GDG initially requested an umbrella review (review of reviews). However, since no eligible reviews were found we included individual studies that were reported in the reviews identified by the search for reviews conducted in PubMed. To ensure that important studies were not missed, we conducted an additional search for individual studies and reviews after submission of the report. This manuscript includes the initial WHO report results as well as the expanded search results. We followed the preferred reporting items for systematic reviews and meta-analyses (PRISMA) guidelines [28, 29], and the PRISMA study flow diagram was used to document the screening process.

\section{Data source and search}

A search for existing systematic reviews was conducted in PubMed for reviews published from 2008 up to November 2019 (Additional file 1, A). An expanded search was conducted in PubMed for individual studies published from January 2010 to March 2020 (Additional file 1 , B). A second expanded search was conducted in PubMed and three additional databases (CINAHL, Embase, SPORTDiscus) for reviews published from 2008 up to July 2020 (Additional file 1, C).

\section{Study selection}

Two reviewers screened all titles and abstracts to identify studies that addressed the present research questions. The full text of each study that potentially met the inclusion criteria was obtained and independently assessed for eligibility by two reviewers. Any disagreements were discussed and when consensus could not be reached, the eligibility of the study was decided following discussion with a third reviewer. We also searched for additional studies in the reference lists of eligible papers and relevant systematic reviews known by the team. All studies were selected according to eligibility criteria below and additional details on eligibility criteria can be found in Additional file 1, D.

\section{Population}

We included studies investigating adults aged 65 years and older. Studies that included younger participants were included if the mean age minus one standard deviation was more than 64 years and/or if participants met the age criteria at follow-up. Studies that recruited participants on the basis of having osteoporosis at baseline were excluded. We followed the WHO definition of osteoporosis on the basis of BMD measurement relative to reference values of young adults of the same sex [2]. No restriction was applied to participants' health status or setting.

\section{Exposure}

The exposure of interest was any volume, duration, frequency, or intensity of physical activity. Studies where participants received multiple interventions were only included if the only difference between the groups was the physical activity intervention. We excluded studies that only used physical activity as a confounding variable as well as studies of multimodal interventions where physical activity was not the main component, or that did not present data on physical activity separately.

\section{Comparison}

We included studies that had no physical activity or lesser volume, duration, frequency, or intensity of physical activity as a comparator.

\section{Outcome}

Our outcome of interest was osteoporosis, including but not limited to BMD from any location (e.g., neck of femur, spine), bone mineral content (BMC), calcium bone index, cortical bone density, and bone quality index. We excluded studies that had fracture as an outcome in the absence of a bone mass measure.

\section{Study design}

We initially searched for systematic reviews and metaanalyses. Since we did not find any eligible systematic review, we identified reviews that included potentially eligible studies and screened all potential studies against our questions. The expanded search was targeted at individual studies that could have been missed by the initial search for reviews. We included individual studies (instead of reviews) that had the following study designs: randomised controlled trials, quasi-randomised controlled trials, prospective cohort studies, and retrospective cohort studies. We excluded cross-sectional and before-and-after studies.

We only included studies published with full-text in English and published in peer-reviewed journals. We excluded grey literature, including unpublished data, abstracts, and conference proceedings. 


\section{Data extraction and quality assessment}

One reviewer extracted information into standardised forms and a second reviewer checked all data. We extracted quantitative estimates for all outcome measures relevant to osteoporosis reported by the included studies.

\section{Physical activity classification}

We used the Prevention of Falls Network Europe (ProFaNE) taxonomy to classify the physical activity and exercise programs in the included trials (Additional file 2) [30]. The programs were classified as primarily involving the following exercise categories: i) gait, balance, coordination and functional task training (referred to as 'balance and functional exercises' for simplicity); ii) strength/resistance training (including power training; using resistance so referred to as 'resistance exercises'); iii) flexibility; iv) three-dimensional (3D) exercise (with Tai Chi or dance subcategories); v) general physical activity (e.g., walking programs); vi) endurance; vii) other kinds of exercise. The taxonomy allows for more than one type of exercise to be delivered within a program. We also considered whether the exercise explicitly included bone loading (e.g., hopping or heel drops) and included this category (i.e., bone loading) as "other kinds of exercise".

\section{Quality assessment}

We assessed the methodological quality of the randomised controlled trials and quasi-randomised trials using the PEDro scale with total scores ranging from 0 to 10 $[31,32]$. We assessed the methodological quality of observational studies using a modified version of the Quality in Prognosis Studies (QUIPS) tool [33] adapted to studies of risk factors. The tool contains six domains and each is categorised as low, moderate or high risk of bias based on explicit criteria (Additional file 3). Overall risk of bias was considered 'low' if four or more domains (including study confounding) were rated as low risk of bias; otherwise, the overall risk of bias was considered 'high'. Two reviewers assessed the risk of bias independently; discrepancies were resolved by a third reviewer.

Using the Grading of Recommendations Assessment, Development and Evaluation (GRADE) framework [34], we examined the quality of primary research and assessed the overall quality of evidence as 'high', 'moderate', 'low' or 'very low' in terms of presence and extent of four factors: risk of bias, inconsistency, imprecision, and publication bias. We did not consider the indirectness criterion because we only included similar studies in terms of population, intervention, comparator and outcome [35]. The quality of the evidence was rated for each outcome. Briefly, we downgraded the evidence by one level for limitation of study design if $>50 \%$ of included trials had a PEDro score $<6 / 10$ [36]. We downgraded the evidence for imprecision if the total number of participants was less than 400 across all studies included in the meta-analysis [37]. We considered the results inconsistent if the heterogeneity between trials was large $\left(\mathrm{I}^{2}>50 \%\right)$ or if there was wide variation of point estimates across the included studies [38]. We assessed publication bias (small study effect) by visual inspection of funnel plots and by performing a sensitivity analysis where we excluded studies with a small sample size $(<$ 50 participants) and we considered whether their removal impacted the pooling of results [39].

\section{Data synthesis and analysis}

We pooled data from all relevant randomised controlled trials comparing physical activity with a control group for the main outcome of each trial. We also performed two additional analyses according to the two most commonly reported outcomes across the included studies. Within each analysis we performed subgroup analyses according to the physical activity classification, as per ProFaNE taxonomy. When data were available for more than one time-point, we extracted data from the time point closest to the end of the intervention. Mean estimates were extracted in the following hierarchical order: mean difference, change score and final score [40]. Where a trial included more than one intervention group, we included each intervention in a separate comparison and divided the number of participants in the control group accordingly to avoid double counting participants in the analyses [40]. We did not include the quasi-randomised trials and the trials investigating clinical populations in the meta-analysis.

We calculated the standardised mean difference (Hedges' g) and 95\% confidence interval (CI) and used random effects meta-analysis models as we considered that a range of true effects was likely but also undertook sensitivity analyses using fixed effect models. Hedges' g was calculated using a combination of data format including mean difference, pre- and post score or change score data (as per individual study's availability) and was standardised using the post-test score standard deviation where available. We used Comprehensive Meta-Analysis (Version 3, Biostat, Englewood NJ).

We undertook meta-regression to investigate the impact of different doses and types of physical activity interventions and study quality using Stata metan and metareg commands (Version 15, College Station, TX). For meta-regression we classified programs with 7800 total minutes (i.e., 150 mins $\times 52$ weeks) or more as high dose programs. Type of physical activity intervention was coded according to the presence of ProFaNE taxonomy categories outlined above: balance/function, bone loading, resistance, multiple exercise and combination of 
multiple and resistance exercise types. We explored the impact of study methodological quality by undertaking meta-regression to compare effects in trials with PEDro scores equal or greater than 6 or below 6 .

\section{Results}

\section{Initial search}

The initial search for systematic reviews and metaanalyses did not identify sufficient evidence to answer the review questions. We screened the full texts of 36 reviews and no eligible reviews were found (Fig. 1). The main reasons for exclusion were reviews including younger participants $(n=34)$, participants with osteoporosis at baseline (management instead of prevention, $n=12$ ), and not investigating whole body physical activity (e.g., whole body vibration, $n=7$ ).

Amongst the 36 reviews which had their full text screened, 25 reviews included potentially eligible studies and their full texts were identified and assessed by two reviewers. We used the same eligibility criteria, but no restriction was applied for publication year of individual studies. We found 36 studies (trials and observational studies) investigating the association between physical activity and prevention of osteoporosis (34 identified from the reviews and 2 from hand searching) [41-76].

\section{Expanded search for individual studies}

The expanded search yielded 772 records and the full texts of 92 records were screened (Fig. 1). A total of 24 studies met the eligibility criteria, 23 identified via PubMed search and one via hand searching. Out of the 24 studies identified, five had already been included in the WHO report $[42,47,66,68,71]$. Therefore, the expanded search found 19 additional studies [77-95].

\section{Expanded search for systematic reviews}

The expanded search for systematic reviews identified a total of 366 reviews. We screened the full texts of 58 reviews and no eligible reviews were found (Fig. 1). Amongst the 58 reviews which had their full text screened, 30 reviews included potentially eligible individual studies and after assessing their full text we included 4 additional studies [96-99], resulting in a total of 59 studies included in this manuscript. The included studies were published between 1980 and 2020. There were 39 randomised controlled trials, 8 quasi-randomised trials and 12 observational studies (8 prospective and 4 retrospective studies). There were three cases where results from the same study were reported across multiple articles $[53,77,81,94,96]$, all of which were included in this review as they reported results for different followup timepoints.

\section{Exposure}

Within the included trials, 40 compared physical activity with a control intervention (Table 1); 11 compared two physical activity programs (Table 2); six trials (Table 3 ) and eight observational studies (Table 4) investigated different doses of physical activity. A total of 12 observational studies were included, seven investigated total physical activity, one leisure-time physical activity (exercise, transportation and sport), and five planned physical activity (four exercise and one sport-tennis) (Table 4).

The included trials comprised a wide range of physical activity and exercise modalities. Following the ProFaNE taxonomy, most studies $(n=19)$ investigated more than one category of exercise (classified as multiple); 11 studies investigated balance and functional exercises, 12 resistance; five endurance; nine investigated a combination of balance and functional exercise or resistance with bone loading; and one 3D exercises (Tai Chi).

\section{Participant characteristics}

Most included studies recruited from the general older population. Studies in which all participants had already been diagnosed with osteoporosis were excluded. Four studies excluded participants with osteoporosis at baseline $[53,93,95,96]$. Three studies included participants on the basis of having some level of frailty [46, 73, 74]; five articles reporting results from two studies included only participants with osteopenia [77, 81, 91, 92, 94]; two studies included only obese participants $[87,88]$; two studies investigated prostate cancer survivors without osteoporosis [93, 95]; one study included participants who had had surgical repair of a hip fracture no more than 16 weeks prior to study entry [43]; and one study included participants with increased risk for falls and fracture [90]. One study investigated lifelong tennis athletes. Twenty-eight studies included only women whereas six investigated only men. Five studies (reported in 8 articles) included participants who were younger than 65 years at study entry, but met the age criteria at follow-up [66, 77, 81, 86, 91, 92, 94, 98].

\section{Outcomes}

The included studies reported results for a range of different outcomes $(n=32)$, and the most common ones were measures of BMD and BMC. We performed an overall assessment of the evidence according to the study's main outcome. If the study did not specify a main outcome, we selected the outcome we considered to be most relevant to the intervention (e.g., whole body for exercises involving the whole body). We selected lumbar spine in preference to hip when both were presented, and the exercise was primarily undertaken in a standing position. Where exercises were mostly performed in non-standing positions (e.g., seated, supine) 


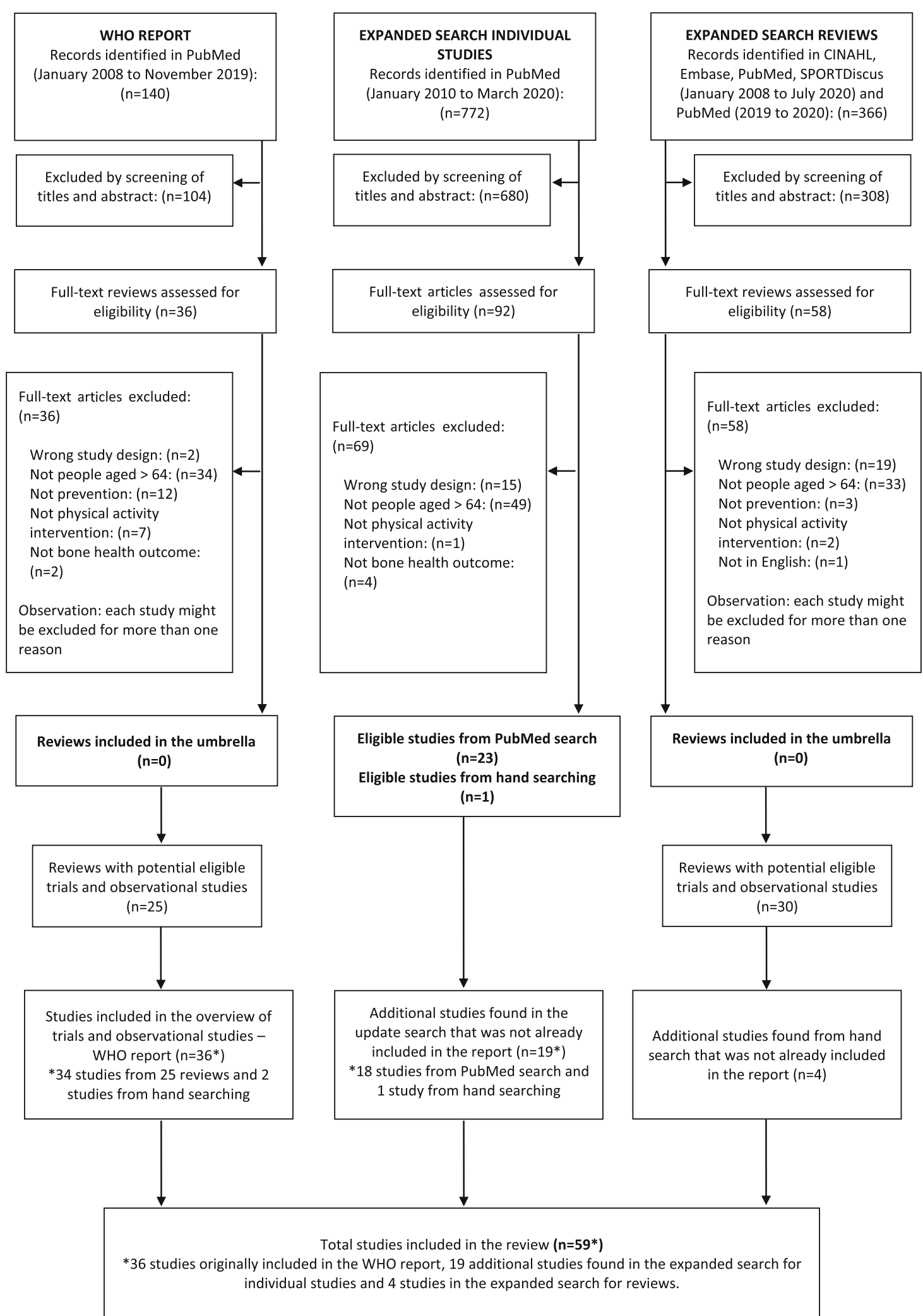

Fig. 1 Flow chart of studies investigating physical activity and osteoporosis prevention in older people included in the WHO report (left size), in the expanded search for individual studies (middle) and expanded search for systematic reviews (right side) 
Table 1 Description of included studies comparing physical activity with a control intervention

\begin{tabular}{|c|c|c|c|c|c|c|c|}
\hline $\begin{array}{l}\text { Reference } \\
\text { PEDro score }\end{array}$ & $\begin{array}{l}\text { Study } \\
\text { design } \\
\text { Allocated/ } \\
\text { Analysed }\end{array}$ & $\begin{array}{l}\text { Participants (n, } \\
\text { age mean (SD), \% } \\
\text { women, setting, } \\
\text { health status) }\end{array}$ & $\begin{array}{l}\text { Intervention } \\
\text { Primary exercise } \\
\text { type according to } \\
\text { ProFANE }^{\mathrm{a}}\end{array}$ & Control $^{b}$ & Outcomes & $\begin{array}{l}\text { Follow } \\
\text { up } \\
\text { (mo) }\end{array}$ & Results \\
\hline $\begin{array}{l}\text { Allison } 2013 \\
5 / 10[41]\end{array}$ & $\begin{array}{l}\mathrm{RCT} \\
50 / 35\end{array}$ & $\begin{array}{l}\text { Setting: } \\
\text { Community; } \\
\text { United Kingdom } \\
\text { Health status: } \\
\text { Healthy } \\
\text { A. High impact } \\
\text { exercise } \\
\boldsymbol{n}=50 \\
\text { (randomised); } 35 \\
\text { (analysed) } \\
\text { Age: } 69.9 \text { (4.0) } \\
\text { Female: } 0 \% \\
\text { B. No exercise } \\
\text { Contralateral leg of } \\
\text { each participant } \\
\text { was used as } \\
\text { control }\end{array}$ & $\begin{array}{l}\text { A. High impact } \\
\text { unilateral exercise } \\
\text { programme (brief } \\
\text { hopping exercise } \\
\text { sessions) } \\
\text { Frequency: } 7 \text { times/ } \\
\text { week } \\
\text { Intensity: } 5 \text { sets of } 10 \\
\text { multidirectional hops } \\
\text { with a 15s rest period. } \\
\text { Encouraged } \\
\text { participants to } \\
\text { continue to hop as } \\
\text { high and as fast as } \\
\text { they could. } \\
\text { Session duration: } \\
\sim 15 \text { min } \\
\text { Delivered by: NR } \\
\text { Duration of the } \\
\text { intervention (wks): } \\
52 \\
\text { Primary exercise } \\
\text { type: Balance and } \\
\text { function including } \\
\text { bone loading } \\
\text { (multidirectional } \\
\text { hopping) }\end{array}$ & $\begin{array}{l}\text { B. No exercises } \\
\text { performed with the } \\
\text { control leg }\end{array}$ & $\begin{array}{l}\text { 1. Femoral neck } \\
\text { BMD } \\
\text { 2. Trochanter BMD } \\
\text { 3. Total hip BMD } \\
\text { 4. Femoral neck } \\
\text { BMC } \\
\text { 5. Trochanter BMC } \\
\text { 6. Total hip BMC }\end{array}$ & 12 & $\begin{array}{l}\text { Final score (mean } \pm \\
\text { SD) } \\
\text { 1. Femoral neck } \\
\text { BMD } \\
\text { A. Exercise: } 0.954 \pm \\
0.017 \\
\text { B. Control: } 0.945 \pm \\
\text { 0.018 } \\
\text { 2. Trochanter BMD } \\
\text { A. Exercise: } 0.923 \pm \\
\text { 0.017 } \\
\text { B. Control: } 0.923 \pm \\
\text { 0.018 } \\
\text { 3. Total hip BMD } \\
\text { A. Exercise: } 1.030 \pm \\
\text { 0.017 } \\
\text { B. Control: } 1.027 \pm \\
\text { 0.018 } \\
\text { 4.Femoral neck } \\
\text { BMC } \\
\text { A. Exercise: } 5.54 \pm \\
\text { 0.13 } \\
\text { B. Control: } 5.49 \pm \\
\text { 0.14 } \\
\text { 5. Trochanter BMC } \\
\text { A. Exercise: } 16.45 \pm \\
\text { 0.54 } \\
\text { B. Control: } 16.49 \pm \\
\text { 0.57 } \\
\text { 6. Total hip BMC: } \\
\text { A. Exercise: } 40.49 \pm \\
\text { 0.91 } \\
\text { B. Control: } 40.35 \pm \\
\text { 0.97 }\end{array}$ \\
\hline $\begin{array}{l}{ }^{\mathrm{d}} \text { Armamento- } \\
\text { Villareal } 2012 \\
7 / 10[88]\end{array}$ & $\begin{array}{l}\text { RCT } \\
107 / 107\end{array}$ & $\begin{array}{l}\text { Setting: } \\
\text { Community; } \\
\text { United States } \\
\text { Health status: } \\
\text { Obese older adults } \\
\text { A. Exercise group } \\
\boldsymbol{n}=26 \\
\text { Age: } 70(0.8) \\
\text { Female: } 61 \% \\
\text { B. Diet group } \\
\text { (weight loss) } \\
\boldsymbol{n}=26 \\
\text { Age: } 70(0.8) \\
\text { Female: } 65 \% \\
\text { C. Diet and } \\
\text { exercise } \\
\boldsymbol{n}=28 \\
\text { Age: } 70(0.8) \\
\text { Female: } 57 \% \\
\text { D. Control } \\
\boldsymbol{n}=27 \\
\text { Age: } 69(0.8) \\
\text { Female: } 67 \%\end{array}$ & $\begin{array}{l}\text { A. Exercise Group } \\
\text { involving aerobic } \\
\text { exercises, progressive } \\
\text { resistance training, } \\
\text { and exercises to } \\
\text { improve flexibility and } \\
\text { balance. } \\
\text { Frequency: } 3 \text { times/ } \\
\text { week } \\
\text { Intensity: aerobic } \\
\text { dance: from } 65 \% \text { of } \\
\text { peak heart rate to } \\
70 \%-85 \% \text { of peak } \\
\text { heart rate; resistance: } \\
\text { from } 1 \text { to } 2 \text { sets at a } \\
\text { resistance of } \\
\text { approximately } 65 \% \text { of } \\
\text { one-repetition max- } \\
\text { imum, with } 8-12 \text { reps } \\
\text { to } 2-3 \text { sets at a resist- } \\
\text { ance of } 80 \% \text { of one- } \\
\text { repetition maximum, } \\
\text { with } 6 \text { to } 8 \text { reps } \\
\text { Session duration: } 90 \\
\text { min } \\
\text { Delivered by: Physical } \\
\text { therapist } \\
\text { Duration of the } \\
\text { intervention (wks): } \\
52 \\
\text { Primary exercise }\end{array}$ & $\begin{array}{l}\text { D. Control - no } \\
\text { intervention or } \\
\text { advice }\end{array}$ & $\begin{array}{l}\text { 1. Femoral neck } \\
\text { BMD } \\
\text { 2. Intertrochanter } \\
\text { BMD } \\
\text { 3. Femoral shaft } \\
\text { BMD }\end{array}$ & 12 & $\begin{array}{l}\text { Change }(\% \pm \text { SD) } \\
\text { 1. Femoral neck } \\
\text { BMD } \\
\text { A. Exercise: } 1.00 \pm \\
0.76 \\
\text { B. Diet: }-2.09 \pm 1.07 \\
\text { C. Diet and exercise: } \\
-0.13 \pm 0.91 \\
\text { D. Control: }-0.08 \pm \\
0.82 \\
\text { 2. Intertrochanter } \\
\text { BMD } \\
\text { A. Exercise: } 1.83 \pm \\
\text { 0.59 } \\
\text { B. Diet: }-2.09 \pm 1.06 \\
\text { C. Diet and exercise: } \\
-1.06 \pm 0.98 \\
\text { D. Control: }-0.18 \pm \\
\text { 0.73 } \\
\text { 3. Femoral shaft } \\
\text { BMD } \\
\text { A. Exercise: } 1.83 \pm \\
\text { 0.59c } \\
\text { B. Diet: }-2.47 \pm 0.51 \\
\text { C. Diet and exercise: } \\
-0.92 \pm 0.83 \\
\text { D. Control: } 0.48 \pm \\
\text { 0.61 }\end{array}$ \\
\hline
\end{tabular}


Table 1 Description of included studies comparing physical activity with a control intervention (Continued)

\begin{tabular}{|c|c|c|c|c|c|c|c|}
\hline $\begin{array}{l}\text { Reference } \\
\text { PEDro score }\end{array}$ & $\begin{array}{l}\text { Study } \\
\text { design } \\
\text { Allocated/ } \\
\text { Analysed }\end{array}$ & $\begin{array}{l}\text { Participants (n, } \\
\text { age mean (SD), \% } \\
\text { women, setting, } \\
\text { health status) }\end{array}$ & $\begin{array}{l}\text { Intervention } \\
\text { Primary exercise } \\
\text { type according to } \\
\text { ProFANE }^{\mathrm{a}}\end{array}$ & Control $^{b}$ & Outcomes & $\begin{array}{l}\text { Follow } \\
\text { up } \\
\text { (mo) }\end{array}$ & Results \\
\hline & & & $\begin{array}{l}\text { type: Multiple } \\
\text { (endurance plus } \\
\text { flexibility plus } \\
\text { resistance plus balance } \\
\text { and function) }\end{array}$ & & & & \\
\hline $\begin{array}{l}\text { Binder } 2004 \\
7 / 10[43]\end{array}$ & $\begin{array}{l}\mathrm{RCT} \\
90 / 78\end{array}$ & $\begin{array}{l}\text { Setting: Hospital; } \\
\text { home care } \\
\text { programme and } \\
\text { community; United } \\
\text { States } \\
\text { Health Status: } \\
\text { People with a } \\
\text { recent proximal } \\
\text { femur fracture } \\
\text { A. Physical } \\
\text { Therapy and } \\
\text { exercise training } \\
\boldsymbol{n}=46 \\
\text { (randomised); } 46 \\
\text { (analysed) } \\
\text { Age: } 80 \text { (7) } \\
\text { Female: } 72 \% \\
\text { B. Control - } \\
\text { Home exercise } \\
\boldsymbol{n}=44 \\
\text { (randomised); } 44 \\
\text { (analysed) } \\
\text { Age: } 81 \text { (8) } \\
\text { Female: } 77 \%\end{array}$ & $\begin{array}{l}\text { A. Supervised physical } \\
\text { therapy and exercise } \\
\text { training involving } \\
\text { flexibility, balance, } \\
\text { coordination, } \\
\text { movement speed and } \\
\text { progressive resistance } \\
\text { exercises. } \\
\text { Frequency: } 3 \text { times/ } \\
\text { week } \\
\text { Intensity: the } \\
\text { resistance training } \\
\text { started from 1-2 sets } \\
\text { of } 6-8 \text { reps each exer- } \\
\text { cise at } 65 \% \text { of } 1 \text { RM; } \\
\text { progressed to } 8-12 \\
\text { reps } 3 \text { sets at } 85 \%- \\
100 \% \text { of initial 1-RM. } \\
\text { Session duration: } 45 \\
\text { to } 90 \text { min } \\
\text { Delivered by: Physical } \\
\text { therapist } \\
\text { Duration of the } \\
\text { intervention (wks): } \\
26 \\
\text { Primary exercise } \\
\text { type: Multiple } \\
\text { (balance/function plus } \\
\text { resistance) }\end{array}$ & $\begin{array}{l}\text { B. Low-intensity } \\
\text { home exercise } \\
\text { programme }\end{array}$ & $\begin{array}{l}\text { 1. Whole body } \\
\text { BMD } \\
\text { 2. Hip BMD }\end{array}$ & 6 & $\begin{array}{l}\text { Final score (mean } \pm \\
\text { SD) } \\
\text { 1. Whole body BMD } \\
\text { A. Physical therapy } \\
\text { and exercise } \\
\text { training: } 1.03 \pm 0.13 \\
\text { B. Home exercise } \\
\text { programme: } 1.00 \pm \\
0.11 \\
\text { 2. Hip BMD } \\
\text { A. Physical therapy } \\
\text { and exercise } \\
\text { training: } 0.64 \pm 0.18 \\
\text { B. Home exercise } \\
\text { programme: } 0.69 \pm \\
0.12 \\
\text { No significant group } \\
\text { x time effects }\end{array}$ \\
\hline $\begin{array}{l}\text { Blumenthal } \\
1991 \\
6 / 10[44]\end{array}$ & $\begin{array}{l}\text { RCT } \\
101 / 84\end{array}$ & $\begin{array}{l}\text { Setting: NR; } \\
\text { United States } \\
\text { Health status: } \\
\text { Healthy } \\
\text { A. Aerobic } \\
\text { Training } \\
\boldsymbol{n}=33 \\
\text { (randomised) } \\
\text { B. Yoga and } \\
\text { flexibility } \\
\boldsymbol{n}=34 \\
\text { (randomised) } \\
\text { C. Control } \\
\boldsymbol{n}=34 \\
\text { (randomised) } \\
\text { Age (whole } \\
\text { sample): } 67 \text { (range: } \\
\text { 60-83) } \\
\text { Female: NR }\end{array}$ & $\begin{array}{l}\text { A. Aerobic training: } \\
\text { Endurance training } \\
\text { involving bicycle } \\
\text { ergometry, brisk } \\
\text { walking/jogging, and } \\
\text { arm ergometry. } \\
\text { Frequency: } 3 \text { times/ } \\
\text { week } \\
\text { Intensity: } 70 \% \text { heart } \\
\text { rate reserve } \\
\text { Session duration: } 60 \\
\text { min } \\
\text { Delivered by: NR } \\
\text { Duration of } \\
\text { intervention (wks): } \\
16 \\
\text { Primary exercise } \\
\text { type: Endurance } \\
\text { training } \\
\text { B. Yoga: Supervised } \\
\text { non-aerobic yoga } \\
\text { programme. } \\
\text { Frequency: at least } 2 \\
\text { times/week } \\
\text { Intensity: NR } \\
\text { Session duration: } 60 \\
\text { min } \\
\text { Delivered by: NR } \\
\text { Duration of } \\
\text { intervention (wks): }\end{array}$ & $\begin{array}{l}\text { C. Waiting list } \\
\text { control: did not } \\
\text { receive any form of } \\
\text { treatment and were } \\
\text { instructed not to } \\
\text { change their } \\
\text { physical activity } \\
\text { habits and } \\
\text { specifically not to } \\
\text { engage in any } \\
\text { aerobic exercise for } \\
\text { the 4-month period. }\end{array}$ & $\begin{array}{l}\text { 1. Distal radius } \\
\mathrm{BMD}\left(\mathrm{mg} / \mathrm{cm}^{2}\right)\end{array}$ & $4,8,14$ & $\begin{array}{l}\text { 1. Distal radius BMD: } \\
\text { no between-group } \\
\text { differences. } \\
\text { Quantitative } \\
\text { estimates not } \\
\text { reported for } \\
\text { between-group } \\
\text { comparisons. } \\
\text { Sub-analysis was } \\
\text { performed } \\
\text { comparing } \\
\text { participants who } \\
\text { decided to continue } \\
\text { to exercise or not } \\
\text { for men and women } \\
\text { separately } \\
\text { (randomisation was } \\
\text { broken for this } \\
\text { analysis): } \\
\text { Female: } \\
\text { Mean } \pm \text { SD at eight } \\
\text { months: } \\
\text { Females who } \\
\text { continued the } \\
\text { programme: } 0.7 \pm \\
0.2 \\
\text { Females who } \\
\text { discontinued the } \\
\text { programme: } 0.8 \pm \\
0.1\end{array}$ \\
\hline
\end{tabular}


Table 1 Description of included studies comparing physical activity with a control intervention (Continued)

\begin{tabular}{|c|c|c|c|c|c|c|c|}
\hline $\begin{array}{l}\text { Reference } \\
\text { PEDro score }\end{array}$ & $\begin{array}{l}\text { Study } \\
\text { design } \\
\text { Allocated/ } \\
\text { Analysed }\end{array}$ & $\begin{array}{l}\text { Participants (n, } \\
\text { age mean (SD), \% } \\
\text { women, setting, } \\
\text { health status) }\end{array}$ & $\begin{array}{l}\text { Intervention } \\
\text { Primary exercise } \\
\text { type according to } \\
\text { ProFANE }^{\mathrm{a}}\end{array}$ & Control $^{b}$ & Outcomes & $\begin{array}{l}\text { Follow } \\
\text { up } \\
\text { (mo) }\end{array}$ & Results \\
\hline & & & $\begin{array}{l}16 \\
\text { Primary exercise } \\
\text { type: Balance and } \\
\text { function }\end{array}$ & & & & $\begin{array}{l}\text { Mean } \pm \text { SD at } \\
\text { fourteen months: } \\
\text { Females who } \\
\text { continued the } \\
\text { programme: } 0.7 \pm \\
0.3 \\
\text { Females who } \\
\text { discontinued the } \\
\text { programme: } 0.9 \pm \\
0.2 \\
\text { Men } \\
\text { Mean } \pm \text { SD at eight } \\
\text { months: } \\
\text { Men who continued } \\
\text { the programme: } 1.2 \\
\pm 0.2 \\
\text { Men who } \\
\text { discontinued the } \\
\text { programme: } 1.1 \pm \\
0.2 \\
\text { Mean } \pm \text { SD at } \\
\text { fourteen months: } \\
\text { Men who continued } \\
\text { the programme: } 1.4 \\
\pm 0.4 \\
\text { Men who } \\
\text { discontinued the } \\
\text { programme: } 1.0 \pm \\
0.3 \\
\text { Between-group } \\
\text { difference: } p<0.05\end{array}$ \\
\hline $\begin{array}{l}\text { Bunout } 2001 \\
4 / 10[45]\end{array}$ & $\begin{array}{l}\text { RCT } \\
149 / 98\end{array}$ & $\begin{array}{l}\text { Setting: } \\
\text { Outpatient clinic; } \\
\text { Chile } \\
\text { Health Status: } \\
\text { Healthy } \\
\text { A. Resistance } \\
\text { training } \\
\boldsymbol{n}=28 \text { randomised; } \\
16 \text { (analysed) } \\
\text { Age: } 74.4 \text { (3.3) } \\
\text { Female: } 75 \% \\
\text { B. Control } \\
\boldsymbol{n}=31 \\
\text { (randomised); } 25 \\
\text { (analysed) } \\
\text { Age: } 74.0 \text { (3.7) } \\
\text { Female: } 48 \% \\
\text { C. } \\
\text { Supplementation } \\
\text { + Resistance } \\
\text { training } \\
\boldsymbol{n}=42 \\
\text { (randomised); } 31 \\
\text { (analysed) } \\
\text { Age: } 73.7(3.0) \\
\text { Female: } 40 \% \\
\text { D. } \\
\text { Supplementation } \\
\boldsymbol{n}=42 \\
\text { (randomised); } 26 \\
\text { (analysed) } \\
\text { Age: } 74.7(3.7) \\
\text { Female: } 62 \%\end{array}$ & $\begin{array}{l}\text { A. Endurance training } \\
\text { consisting of exercise } \\
\text { for upper and lower } \\
\text { body, respiratory } \\
\text { muscle training and } \\
\text { walking. } \\
\text { Frequency: } 2 \text { times/ } \\
\text { week } \\
\text { Intensity: Graded by a } \\
\text { coach using the Borg } \\
\text { scale } \\
\text { Session duration: } 60 \\
\text { min } \\
\text { Delivered by: } \\
\text { Specialised coach } \\
\text { Duration of the } \\
\text { intervention (wks): } \\
78 \\
\text { Primary exercise } \\
\text { type: Multiple } \\
\text { (balance and function } \\
\text { plus endurance) }\end{array}$ & B. No training & $\begin{array}{l}\text { 1. Whole body } \\
\text { BMD } \\
\text { 2. Whole body } \\
\text { BMC }\end{array}$ & 18 & $\begin{array}{l}\text { 1. Whole body BMD } \\
\text { Decreased } \\
\text { significantly in all } \\
\text { groups ( } p=0.006 \text { ), } \\
\text { but the decline was } \\
\text { less marked in the } \\
\text { strength training } \\
\text { combined with } \\
\text { nutritional } \\
\text { supplements } \\
\text { compared with the } \\
\text { other groups } \\
\text { (statistically } \\
\text { significant). } \\
\text { Results reported in a } \\
\text { graph and not } \\
\text { possible to extract } \\
\text { quantitative } \\
\text { estimates. } \\
\text { 2. Whole body BMC: } \\
\text { NR }\end{array}$ \\
\hline
\end{tabular}


Table 1 Description of included studies comparing physical activity with a control intervention (Continued)

\begin{tabular}{|c|c|c|c|c|c|c|c|}
\hline $\begin{array}{l}\text { Reference } \\
\text { PEDro score }\end{array}$ & $\begin{array}{l}\text { Study } \\
\text { design } \\
\text { Allocated/ } \\
\text { Analysed }\end{array}$ & $\begin{array}{l}\text { Participants (n, } \\
\text { age mean (SD), \% } \\
\text { women, setting, } \\
\text { health status) }\end{array}$ & $\begin{array}{l}\text { Intervention } \\
\text { Primary exercise } \\
\text { type according to } \\
\text { ProFANE }^{\mathrm{a}}\end{array}$ & Control $^{b}$ & Outcomes & $\begin{array}{l}\text { Follow } \\
\text { up } \\
(\mathrm{mo})\end{array}$ & Results \\
\hline $\begin{array}{l}\text { De Jong } \\
2000 \\
5 / 10[46]\end{array}$ & $\begin{array}{l}\text { RCT } \\
217 / 143\end{array}$ & $\begin{array}{l}\text { Setting: } \\
\text { Community; } \\
\text { Netherlands } \\
\text { Health status: } \\
\text { People with frailty } \\
\text { and BMl } \leq 25 \\
\text { A. Exercise } \\
\boldsymbol{n}=55 \\
\text { (randomised); } 36 \\
\text { (analysed) } \\
\text { Age: } 76.5 \text { (4.6) } \\
\text { Female: } 69 \% \\
\text { B. Control } \\
\boldsymbol{n}=44 \\
\text { (randomised); } 33 \\
\text { (analysed) } \\
\text { Age: } 78.8 \text { (6.7) } \\
\text { Female: } 67 \% \\
\text { C. Exercise + } \\
\text { nutrition } \\
\boldsymbol{n}=60 \\
\text { (randomised); } 39 \\
\text { (analysed) } \\
\text { Age: } 79.8 \text { (5.8) } \\
\text { Female: 74\% } \\
\text { D. Nutrition } \\
\boldsymbol{n}=58 \\
\text { (randomised); } 35 \\
\text { (analysed) } \\
\text { Age: } 79.6 \text { (5.0) } \\
\text { Female: } 69 \%\end{array}$ & $\begin{array}{l}\text { A. Supervised group- } \\
\text { based exercise } \\
\text { programme involving } \\
\text { muscle strength, co- } \\
\text { ordination, flexibility, } \\
\text { speed, endurance with } \\
\text { use of ropes, weights } \\
\text { and elastic bands. } \\
\text { Frequency: } 2 \text { times/ } \\
\text { week } \\
\text { Intensity: Moderate to } \\
\text { high; } 7 \text { of a 10-point } \\
\text { Borg scale } \\
\text { Session duration: } 45 \\
\text { min } \\
\text { Delivered by: Skilled } \\
\text { teachers and } \\
\text { supervisor } \\
\text { Duration of the } \\
\text { intervention (wks): } \\
17 \\
\text { Primary exercise } \\
\text { type: Balance and } \\
\text { function }\end{array}$ & $\begin{array}{l}\text { B. Social } \\
\text { programme } \\
\text { involving creative } \\
\text { and social activities } \\
\text { as well as } \\
\text { educational sessions. }\end{array}$ & $\begin{array}{l}\text { 1. Whole body } \\
\text { BMD }\end{array}$ & 4.5 & $\begin{array}{l}\text { Change score (mean } \\
\text { change } \pm \text { SD) } \\
\text { 1. Whole body BMD } \\
\text { A. Exercise: } 0.000 \pm \\
0.022 \\
\text { B. Control: }-0.003 \pm \\
0.018 \\
\text { C. Combination } \\
\text { group: } 0.003 \pm 0.023 \\
\text { D. Nutrition group: } \\
0.006 \pm 0.014 \\
\text { No between-group } \\
\text { differences in the } \\
\text { relevant compari- } \\
\text { sons to this review } \\
\text { (ie, exercise vs con- } \\
\text { trol and combin- } \\
\text { ation vs nutrition) }\end{array}$ \\
\hline $\begin{array}{l}\text { Duckham } \\
2015 \\
6 / 10[47]\end{array}$ & $\begin{array}{l}\text { RCT } \\
319 / 283\end{array}$ & $\begin{array}{l}\text { Setting: General } \\
\text { practice; United } \\
\text { Kingdom } \\
\text { Health status: } \\
\text { Healthy } \\
\text { A. Home based } \\
\text { exercise (OEP) } \\
\boldsymbol{n}=88 \\
\text { (randomised); } 75 \\
\text { (analysed) } \\
\text { Age: } 71.4 \text { (4.9) } \\
\text { Female: } 68 \% \\
\text { B. Community } \\
\text { based exercise } \\
\text { (FaME) } \\
\boldsymbol{n}=105 \\
\text { (randomised); } 94 \\
\text { (analysed) } \\
\text { Age: } 71.8 \text { (5.5) } \\
\text { Female: } 60 \% \\
\text { C. Control: Usual } \\
\text { care } \\
\boldsymbol{n}=126 \\
\text { (randomised); } 114 \\
\text { (analysed) } \\
\text { Age: } 72.2 \text { (5.5) } \\
\text { Female: } 54 \%\end{array}$ & $\begin{array}{l}\text { A. OEP: Home } \\
\text { exercise programme } \\
\text { consisting of leg } \\
\text { strengthening, balance } \\
\text { exercise, and walking. } \\
\text { Frequency: } 3 \\
\text { sessions/week of } \\
\text { home exercise; at least } \\
2 \text { sessions/week of } \\
\text { walking } \\
\text { Intensity: Walking } \\
\text { moderate pace } \\
\text { Session duration: } 30 \\
\text { min/home exercise } \\
\text { session, and } 30 \text { min/ } \\
\text { walking session } \\
\text { Delivered by: Trial } \\
\text { research staff in the } \\
\text { one-off training } \\
\text { Duration of the } \\
\text { intervention (wks): } \\
24 \\
\text { Primary exercise } \\
\text { type: Balance and } \\
\text { function } \\
\text { B. FaME: Falls and } \\
\text { exercise management } \\
\text { programme involving } \\
\text { progressive resistance } \\
\text { training, flexibility } \\
\text { training, functional } \\
\text { floor skill and adapted } \\
\text { Tai Chi. Additionally, } \\
\text { FaME intervention }\end{array}$ & $\begin{array}{l}\text { C. Usual care } \\
\text { Participants not } \\
\text { offered the FaME or } \\
\text { OEP programmes }\end{array}$ & $\begin{array}{l}\text { 1. Femoral neck } \\
\text { BMD } \\
\text { 2. Trochanter BMD } \\
\text { 3. Total hip BMD } \\
\text { 4. Upper neck } \\
\text { BMD } \\
\text { 5. Lumbar spine } \\
\text { BMD } \\
\text { 6. Distal radius } \\
\text { BMD } \\
\text { 7. Whole body } \\
\text { BMD } \\
\text { 8. Whole body } \\
\text { BMC }\end{array}$ & 6 & 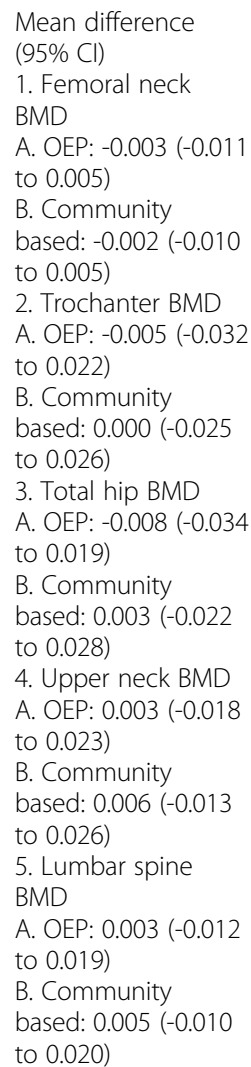 \\
\hline
\end{tabular}


Table 1 Description of included studies comparing physical activity with a control intervention (Continued)

\begin{tabular}{|c|c|c|c|c|c|c|c|}
\hline $\begin{array}{l}\text { Reference } \\
\text { PEDro score }\end{array}$ & $\begin{array}{l}\text { Study } \\
\text { design } \\
\text { Allocated/ } \\
\text { Analysed }\end{array}$ & $\begin{array}{l}\text { Participants ( } \mathrm{n} \text {, } \\
\text { age mean (SD), \% } \\
\text { women, setting, } \\
\text { health status) }\end{array}$ & $\begin{array}{l}\text { Intervention } \\
\text { Primary exercise } \\
\text { type according to } \\
\text { ProFANE }^{\mathrm{a}}\end{array}$ & Control $^{\mathbf{b}}$ & Outcomes & $\begin{array}{l}\text { Follow } \\
\text { up } \\
\text { (mo) }\end{array}$ & Results \\
\hline & & & $\begin{array}{l}\text { included home } \\
\text { exercise based on EOP } \\
\text { and walking. } \\
\text { Frequency: } 3-5 \text { times/ } \\
\text { week [One exercise } \\
\text { class, two home exer- } \\
\text { cise session and at } \\
\text { least two sessions of } \\
\text { walking per week] } \\
\text { Intensity: walking at } \\
\text { moderate pace } \\
\text { Session duration: } 60 \\
\text { min/exercise class; } 30 \\
\text { min/home exercise } \\
\text { session; } 30 \text { min/ } \\
\text { walking session } \\
\text { Delivered by: Postural } \\
\text { stability instructor } \\
\text { Duration of the } \\
\text { intervention (wks): } \\
24 \\
\text { Primary exercise } \\
\text { type: Balance and } \\
\text { function }\end{array}$ & & & & $\begin{array}{l}\text { 6. Distal radius } \\
\text { A. OEP: } 0.001(-0.008 \\
\text { to 0.010) } \\
\text { B. Community } \\
\text { based: }-0.009(-0.018 \\
\text { to }-0.000)^{c} \\
\text { 7. Whole body BMD } \\
\text { A. OEP: } 0.003(-0.002 \\
\text { to 0.008) } \\
\text { B. Community } \\
\text { based: }-0.003 \text { (-0.007 } \\
\text { to 0.002) } \\
\text { 8. Whole body BMC } \\
\text { A. OEP: } 0.8 \text { (-22.0 to } \\
\text { 23.6) } \\
\text { B. Community } \\
\text { based: }-6.6(-27.9 \text { to } \\
\text { 14.7) }\end{array}$ \\
\hline $\begin{array}{l}\text { Englund } \\
2005 \\
5 / 10[48]\end{array}$ & $\begin{array}{l}\text { RCT } \\
48 / 40\end{array}$ & $\begin{array}{l}\text { Setting: } \\
\text { Community; } \\
\text { Sweden } \\
\text { Health status: } \\
\text { Healthy } \\
\text { A. Exercise } \\
\text { (COMB) } \\
\boldsymbol{n}=24 \\
\text { (randomised); } 21 \\
\text { (analysed) } \\
\text { Age: } 72.8 \text { (3.6) } \\
\text { B. Control } \\
\boldsymbol{n}=24 \\
\text { (randomised); } 19 \\
\text { (analysed) } \\
\text { Age:73.2 (4.9) } \\
\text { Female: } 100 \%\end{array}$ & $\begin{array}{l}\text { A. Supervised exercise } \\
\text { programme involving } \\
\text { a combination of } \\
\text { strengthening, aerobic, } \\
\text { balance and } \\
\text { coordination exercises } \\
\text { Frequency: } 2 \text { times/ } \\
\text { week } \\
\text { Intensity: } 2 \text { sets of 8- } \\
12 \text { reps (strengthening } \\
\text { exercise) } \\
\text { Session duration: } 50 \\
\text { min } \\
\text { Delivered by: } \\
\text { Physiotherapist } \\
\text { Duration of the } \\
\text { intervention (wks): } \\
47 \\
\text { Primary exercise } \\
\text { type: Multiple } \\
\text { (balance and function } \\
\text { plus resistance plus } \\
\text { endurance) }\end{array}$ & B. No training & $\begin{array}{l}\text { 1. Lumbar Spine } \\
\text { BMD } \\
\text { 2. Femoral neck } \\
\text { BMD } \\
\text { 3. Trochanter BMD } \\
\text { 4. Ward's triangle } \\
\text { BMD } \\
\text { 5. Whole body } \\
\text { BMD } \\
\text { 6. Arms BMD } \\
\text { 7. Whole body } \\
\text { BMC }\end{array}$ & 12 & $\begin{array}{l}\text { Mean difference } \\
\text { (95\% CI) (on \% } \\
\text { changes) } \\
\text { 1. Lumbar spine } \\
\text { BMD: } 2.1 \text { (-0.4 to 3.4) } \\
\text { 2. Femoral neck } \\
\text { BMD: } 0 \text { (-3.8 to 2.6) } \\
\text { 3. Trochanter BMD: } \\
\text { 3.4 (-1.2 to } 7.3 \text { ) } \\
\text { 4. Ward's triangle } \\
\text { BMD: } 2.2 \text { (1.8 to } \\
\text { 12.9) } \\
\text { 5. Whole body BMD: } \\
\text { 0.1 (-1.3 to 2.2) } \\
\text { 6. Arms BMD: } 0 \text { (-1.9 } \\
\text { to 2.8) } \\
\text { 7. Whole body BMC: } \\
\text { 1.3 (-0.3 to 3.1) }\end{array}$ \\
\hline $\begin{array}{l}\text { Helge } 2014 \\
5 / 10[50]\end{array}$ & $\begin{array}{l}\text { RCT } \\
27 / 23\end{array}$ & $\begin{array}{l}\text { Setting: } \\
\text { Community; } \\
\text { Denmark } \\
\text { Health status: } \\
\text { Healthy } \\
\text { A. Football group } \\
\boldsymbol{n}=9 \text { (randomised); } \\
9 \text { (analysed) } \\
\text { Age: } 68.0 \text { (4.0) } \\
\text { B. Resistance } \\
\text { training } \\
\boldsymbol{n}=9 \text { (randomised); } \\
8 \text { (analysed) } \\
\text { Age: } 69.1 \text { (3.1) } \\
\text { C. Control } \\
\boldsymbol{n}=8 \text { (randomised); } \\
6 \text { (analysed) }\end{array}$ & $\begin{array}{l}\text { A. Football group: } \\
\text { Supervised progressive } \\
\text { football training } \\
\text { Frequency: } 1.7(0.3) \\
\text { times/week (range: } \\
1.2-2.2 \text { ) } \\
\text { Intensity: } 82 \% \text { of } \\
\text { maximum heart rate } \\
\text { (range } 64 \text { to } 90 \% \text { ) } \\
\text { Session duration: } 45 \\
\text { to } 60 \text { min } \\
\text { Delivered by: NR } \\
\text { Duration of the } \\
\text { intervention (wks): } \\
52 \\
\text { Primary exercise } \\
\text { type: Balance and }\end{array}$ & C. Inactive control & $\begin{array}{l}\text { 1. Whole body } \\
\text { BMD } \\
\text { 2. Right femoral } \\
\text { neck BMD } \\
\text { 3. Left femoral } \\
\text { neck BMD } \\
\text { 4. Right femoral } \\
\text { shaft BMD } \\
\text { 5. Left femoral } \\
\text { shaft BMD } \\
\text { 6. Total right } \\
\text { proximal femur } \\
\text { BMD } \\
\text { 7. Total left } \\
\text { proximal femur } \\
\text { BMD }\end{array}$ & 12 & $\begin{array}{l}\text { Final score (mean } \pm \\
\text { SE) } \\
\text { 1. Whole body BMD } \\
\text { A. Football: } 1.211 \pm \\
0.036 \\
\text { B. Resistance: } 1.225 \\
\pm 0.024 \\
\text { C. Control: } 1.268 \pm \\
\text { 0.030 } \\
\text { 2. Right femoral } \\
\text { neck BMD } \\
\text { A. Football: } 0.921 \pm \\
0.034 \\
\text { B. Resistance: } 1.000 \\
\pm 0.042 \\
\text { C. Control: } 1.008 \pm \\
0.063\end{array}$ \\
\hline
\end{tabular}


Table 1 Description of included studies comparing physical activity with a control intervention (Continued)

\begin{tabular}{|c|c|c|c|c|c|c|c|}
\hline $\begin{array}{l}\text { Reference } \\
\text { PEDro score }\end{array}$ & $\begin{array}{l}\text { Study } \\
\text { design } \\
\text { Allocated/ } \\
\text { Analysed }\end{array}$ & $\begin{array}{l}\text { Participants (n, } \\
\text { age mean (SD), \% } \\
\text { women, setting, } \\
\text { health status) }\end{array}$ & $\begin{array}{l}\text { Intervention } \\
\text { Primary exercise } \\
\text { type according to } \\
\text { ProFANE }^{\mathrm{a}}\end{array}$ & Control $^{\mathbf{b}}$ & Outcomes & $\begin{array}{l}\text { Follow } \\
\text { up } \\
\text { (mo) }\end{array}$ & Results \\
\hline & & $\begin{array}{l}\text { Age: } 67.4(2.7) \\
\text { Female: } 0 \%\end{array}$ & $\begin{array}{l}\text { function (football) } \\
\text { B. Resistance } \\
\text { training: Progressive } \\
\text { resistance training for } \\
\text { core and upper and } \\
\text { lower body } \\
\text { Frequency: } 1.9 \text { (0.2) } \\
\text { times/week (range: } \\
1.4-2.2 \text { ) } \\
\text { Intensity: started from } \\
3 \text { sets of } 16-20 \text { RM to } \\
4 \text { sets of } 8 \text { RM } \\
\text { Session duration: } 45 \\
\text { to } 60 \text { min } \\
\text { Delivered by: NR } \\
\text { Duration of the } \\
\text { intervention (wks): } \\
52 \\
\text { Primary exercise } \\
\text { type: Resistance } \\
\text { (seated) }\end{array}$ & & & & 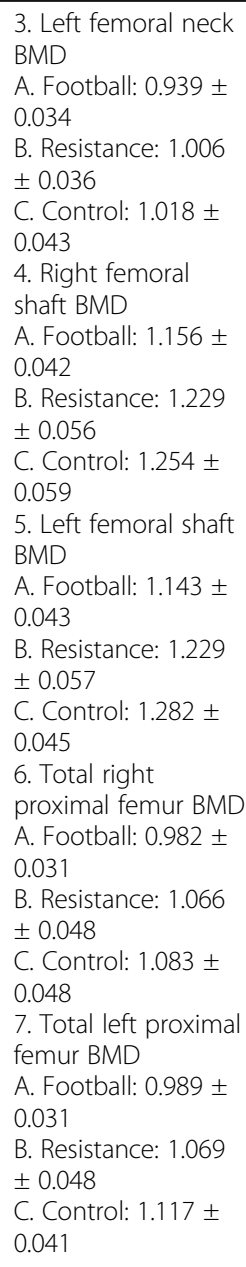 \\
\hline $\begin{array}{l}\text { Jessup 2003 } \\
5 / 10[52]\end{array}$ & $\begin{array}{l}\text { RCT } \\
18 / 16\end{array}$ & $\begin{array}{l}\text { Setting: } \\
\text { Retirement } \\
\text { Community; } \\
\text { United States } \\
\text { Health Status: } \\
\text { Healthy } \\
\text { A. Multi- } \\
\text { component } \\
\text { intervention } \\
\boldsymbol{n}=9 \text { (randomised); } \\
8 \text { (analysed) } \\
\text { Age: } 69.1 \text { (2.8) } \\
\text { B. Control } \\
\boldsymbol{n}=9 \text { (randomised); } \\
8 \text { (analysed) } \\
\text { Age: } 69.4(4.2) \\
\text { Female: } 100 \%\end{array}$ & $\begin{array}{l}\text { A. Supervised exercise } \\
\text { programme involving } \\
\text { resistance training, } \\
\text { load-bearing walking } \\
\text { with use of weights } \\
\text { vest, stair-climbing, } \\
\text { and balance training. } \\
\text { Frequency: } 3 \text { times/ } \\
\text { week } \\
\text { Intensity: } 8-10 \text { reps of } \\
50 \% \text { of } 1 \text { RM, pro- } \\
\text { gressed to } 75 \% \text { of } \\
1 \text { RM (resistance } \\
\text { training } \\
\text { Session duration: } 60 \\
\text { to } 90 \text { min exercise } \\
\text { training session; } 30 \text { to } \\
45 \text { min walking } \\
\text { Delivered by: Co- } \\
\text { investigator and/or re- } \\
\text { search assistant } \\
\text { Duration of the } \\
\text { intervention (wks): } \\
32 \text { weeks } \\
\text { Primary exercise }\end{array}$ & B. Control & $\begin{array}{l}\text { 1. Femoral neck } \\
\text { BMD } \\
\text { 2. Lumbar spine } \\
\text { BMD }\end{array}$ & 8 & $\begin{array}{l}\text { Change score } \\
\text { (ANCOVA, p-value) } \\
\text { 1. Femoral neck } \\
\text { BMD } \\
\text { A. Exercise: } 1.7 \\
\text { B. Control: }-0.04 \\
\text { F }(1,15)=7.38, P= \\
0.016 \\
\text { 2. Lumbar spine } \\
\text { BMD } \\
\text { A. Exercise: } 0.11 \\
\text { B. Control: }-0.003 \\
\text { F }(1,15)=2.70, P= \\
0.121 \\
\text { Final score (mean } \pm \\
\text { SD) } \\
\text { 1. Femoral neck } \\
\text { BMD } \\
\text { A. Exercise: } 0.74 \pm \\
0.05 \\
\text { B. Control: } 0.74 \pm \\
\text { 0.13 } \\
\text { 2. Lumbar spine } \\
\text { BMD } \\
\text { A. Exercise: } 0.88 \pm\end{array}$ \\
\hline
\end{tabular}


Table 1 Description of included studies comparing physical activity with a control intervention (Continued)

\begin{tabular}{|c|c|c|c|c|c|c|c|}
\hline $\begin{array}{l}\text { Reference } \\
\text { PEDro score }\end{array}$ & $\begin{array}{l}\text { Study } \\
\text { design } \\
\text { Allocated/ } \\
\text { Analysed }\end{array}$ & $\begin{array}{l}\text { Participants (n, } \\
\text { age mean (SD), \% } \\
\text { women, setting, } \\
\text { health status) }\end{array}$ & $\begin{array}{l}\text { Intervention } \\
\text { Primary exercise } \\
\text { type according to } \\
\text { ProFANE }^{\mathrm{a}}\end{array}$ & Control $^{b}$ & Outcomes & $\begin{array}{l}\text { Follow } \\
\text { up } \\
\text { (mo) }\end{array}$ & Results \\
\hline & & & $\begin{array}{l}\text { type: Multiple } \\
\text { (balance and function } \\
\text { plus resistance plus } \\
\text { endurance) }\end{array}$ & & & & $\begin{array}{l}0.08 \\
\text { B. Control: } 1.14 \pm \\
0.32\end{array}$ \\
\hline $\begin{array}{l}\text { Karinkanta } \\
20079 \\
7 / 10[53]\end{array}$ & $\begin{array}{l}\text { RCT } \\
149 / 144\end{array}$ & $\begin{array}{l}\text { Setting: } \\
\text { Community; } \\
\text { Finland } \\
\text { Health Status: } \\
\text { healthy and } \\
\text { excluded } \\
\text { participants with } \\
\text { osteoporosis } \\
\text { A. Balance- } \\
\text { jumping training } \\
\boldsymbol{n}= \\
\text { 37(randomised); } 35 \\
\text { (analysed) } \\
\text { Age: } 72.9 \text { (2.3) } \\
\text { B. Resistance } \\
\text { training } \\
\boldsymbol{n}=37 \\
\text { (randomised); } \\
\text { 37(analysed) } \\
\text { Age: } 72.7 \text { (2.5) } \\
\text { C. Combined } \\
\text { Balance-jumping } \\
\text { and resistance } \\
\text { training } \\
\boldsymbol{n}=38 \\
\text { (randomised); } 36 \\
\text { (analysed) } \\
\text { Age: } 72.9 \text { (2.2) } \\
\text { D. Control } \\
\boldsymbol{n}=37 \\
\text { (randomised); } 36 \\
\text { (analysed); } \\
\text { Age: } 72.0 \text { (2.1) } \\
\text { Female: } 100 \% \\
\text { (a) }\end{array}$ & $\begin{array}{l}\text { A. Balance-jumping } \\
\text { training: Balance } \\
\text { training including } \\
\text { static and dynamic } \\
\text { balance exercise, } \\
\text { agility training, impact } \\
\text { exercises and changes } \\
\text { of direction exercise. } \\
\text { Intensity: NR } \\
\text { Primary exercise } \\
\text { type: Balance and } \\
\text { function including } \\
\text { bone loading (jumps) } \\
\text { B. Resistance } \\
\text { training: Tailored } \\
\text { progressive resistance } \\
\text { training programme } \\
\text { for large muscle } \\
\text { groups. } \\
\text { Intensity: Initially } 2 \\
\text { sets of 10-15 reps at } \\
\text { intensity } 50-60 \% \text { of } \\
\text { 1RM, progressed to } 3 \\
\text { sets of 8-10 reps at } 75- \\
80 \% \text { of } 1 \text { RM. Rate of } \\
\text { perceived exertion: } \\
\text { above } 18 \text { out of } 20 \\
\text { Primary exercise } \\
\text { type: Resistance } \\
\text { C. Combined } \\
\text { Balance-jumping and } \\
\text { resistance training: A } \\
\text { combination of A \& B } \\
\text { on alternate weeks. } \\
\text { Primary exercise } \\
\text { type: Multiple } \\
\text { (balance and function } \\
\text { plus resistance) } \\
\text { For all exercise groups: } \\
\text { Frequency: } 3 \text { times/ } \\
\text { week } \\
\text { Session duration: } 50 \\
\text { min } \\
\text { Delivered by: Exercise } \\
\text { leaders } \\
\text { Duration of the } \\
\text { intervention (wks): } \\
\text { 52 }\end{array}$ & $\begin{array}{l}\text { D. Control: maintain } \\
\text { their pre-study level } \\
\text { of physical activity } \\
\text { during the 12- } \\
\text { month trial }\end{array}$ & $\begin{array}{l}\text { 1. Femoral neck } \\
\text { BMC } \\
\text { 2. Distal tibia } \\
\text { trabecular density } \\
\left(\mathrm{mg} / \mathrm{cm}^{3}\right)\end{array}$ & 12 & $\begin{array}{l}\text { Final score (mean } \pm \\
\text { SD) } \\
\text { 1. Femoral neck } \\
\text { BMC } \\
\text { A. Balance: } 2.73 \pm \\
0.40 \\
\text { B. Resistance: } 2.71 \pm \\
0.33 \\
\text { C. Combined: } 2.65 \pm \\
0.29 \\
\text { D. Control: } 2.67 \pm \\
0.44 \\
\text { 2. Distal tibia } \\
\text { trabecular density } \\
\text { (mg/cm }{ }^{3} \text { ) } \\
\text { A. Balance: } 224 \pm 34 \\
\text { B. Resistance: } 219 \pm \\
\text { 26 } \\
\text { C. Combined: } 215 \pm \\
\text { 39 } \\
\text { D. Control: } 226 \pm 33\end{array}$ \\
\hline $\begin{array}{l}{ }^{\text {eKarinkanta }} \\
20099 \\
5 / 10[98]\end{array}$ & $\begin{array}{l}\text { RCT } \\
149 / 126\end{array}$ & $\begin{array}{l}\text { Setting: } \\
\text { Community; } \\
\text { Finland } \\
\text { Health Status: } \\
\text { Healthy and } \\
\text { excluded } \\
\text { participants with } \\
\text { osteoporosis } \\
\text { A. Balance } \\
\text { jumping training } \\
\text { group } \\
\boldsymbol{n}=37\end{array}$ & $\begin{array}{l}\text { A. Balance-jumping } \\
\text { training: Balance } \\
\text { training (static and } \\
\text { dynamic), agility } \\
\text { training, impact } \\
\text { exercises and changes } \\
\text { of direction exercise. } \\
\text { Intensity: NR } \\
\text { Primary exercise } \\
\text { type: Balance and } \\
\text { function including } \\
\text { bone loading (jumps) }\end{array}$ & $\begin{array}{l}\text { D. Control: maintain } \\
\text { their pre-study level } \\
\text { of physical activity }\end{array}$ & $\begin{array}{l}\text { 1. Femoral neck } \\
\text { section moduls (Z) } \\
\left(\mathrm{mm}^{3}\right) \\
\text { 2. Tibia midshaft } \\
\text { desnity-weighted } \\
\text { polar section } \\
\text { modulus (BSI) } \\
\left(\mathrm{mm}^{3}\right)\end{array}$ & 24 & $\begin{array}{l}\text { \% Mean difference } \\
\text { compared to control } \\
(95 \% \text { Cl) } \\
\text { 1. Femoral neck Z } \\
\text { A. Balance: } 3.6(-0.8 \\
\text { to } 8.2) \\
\text { B. Resistance: } 3.5 \\
\text { (-0.8 to } 8.1) \\
\text { C. Combined: } 0.3 \\
\text { (-4.0 to } 4.8) \\
\text { 2. Tibia midshaft BSI } \\
\text { A. Balance: } 0.2(-1.1\end{array}$ \\
\hline
\end{tabular}


Table 1 Description of included studies comparing physical activity with a control intervention (Continued)

\begin{tabular}{|c|c|c|c|c|c|c|c|}
\hline $\begin{array}{l}\text { Reference } \\
\text { PEDro score }\end{array}$ & $\begin{array}{l}\text { Study } \\
\text { design } \\
\text { Allocated/ } \\
\text { Analysed }\end{array}$ & $\begin{array}{l}\text { Participants (n, } \\
\text { age mean (SD), \% } \\
\text { women, setting, } \\
\text { health status) }\end{array}$ & $\begin{array}{l}\text { Intervention } \\
\text { Primary exercise } \\
\text { type according to } \\
\text { ProFANE }^{\mathrm{a}}\end{array}$ & Control $^{\mathbf{b}}$ & Outcomes & $\begin{array}{l}\text { Follow } \\
\text { up } \\
\text { (mo) }\end{array}$ & Results \\
\hline & & $\begin{array}{l}\text { (randomised); } 33 \\
\text { (analysed) } \\
\text { Age: } 72.9(2.3) \\
\text { B. Resistance } \\
\text { training group } \\
\boldsymbol{n}=37 \\
\text { (randomised); } 34 \\
\text { (analysed) } \\
\text { Age: } 72.7(2.5) \\
\text { C. Combined } \\
\text { resistance and } \\
\text { balance jumping } \\
\text { training group } \\
\boldsymbol{n}=38 \\
\text { (randomised); } 32 \\
\text { (analysed) } \\
\text { Age: } 72.9(2.2) \\
\text { D. Non-training } \\
\text { control group } \\
\boldsymbol{n}=37 \\
\text { (randomised); } \\
27 \text { (analysed) } \\
\text { Age: } 72.0 \text { (2.1) } \\
\text { Female: } 100 \%\end{array}$ & $\begin{array}{l}\text { B. Resistance training: } \\
\text { Tailored progressive } \\
\text { resistance for large } \\
\text { muscle groups. } \\
\text { Intensity: Initially } 2 \\
\text { sets of 10-15 reps at } \\
\text { intensity } 50-60 \% \text { of } \\
1 \text { RM, progressed to } 3 \\
\text { sets of 8-10 reps at } 75- \\
80 \% \text { of } 1 \text { RM. Rate of } \\
\text { perceived exertion: } \\
\text { above } 18 \text { out of } 20 \\
\text { Primary exercise } \\
\text { type: Resistance } \\
\text { C. Combined } \\
\text { Balance-jumping and } \\
\text { resistance training: A } \\
\text { combination of A \& B } \\
\text { on alternate weeks. } \\
\text { Primary exercise } \\
\text { type: Multiple } \\
\text { (balance and function } \\
\text { plus resistance } \\
\text { including bone } \\
\text { loading) } \\
\text { For all exercise groups: } \\
\text { Frequency: } 3 \text { times/ } \\
\text { week } \\
\text { Session duration: } 50 \\
\text { min } \\
\text { Delivered by: Exercise } \\
\text { leaders } \\
\text { Duration of the } \\
\text { intervention (wks): } \\
52\end{array}$ & & & & $\begin{array}{l}\text { to } 1.6) \\
\text { B. Resistance: } 0.3 \\
(-1.0 \text { to } 1.6) \\
\text { C. Combined:0.6 } \\
(-0.7 \text { to } 1.9)\end{array}$ \\
\hline $\begin{array}{l}{ }^{d} \text { Kemmler } \\
2012 \S \\
4 / 10[94]\end{array}$ & $\begin{array}{l}\text { Quasi- } \\
\text { randomised } \\
\text { trial } \\
137 / 85\end{array}$ & $\begin{array}{l}\text { Setting: } \\
\text { Community; } \\
\text { Germany } \\
\text { Health Status: } \\
\text { Osteopenia } \\
\text { A. Exercise group } \\
\boldsymbol{n}=86 \\
\text { (randomised); } 41 \\
\text { (analysed) } \\
\text { Age: } 55.0 \text { (3.4) } \\
\text { B. Control-no } \\
\text { training } \\
\boldsymbol{n}=51 \\
\text { (randomised); } 44 \\
\text { (analysed) } \\
\text { Age: } 55.8 \text { (3.1) } \\
\text { Female: } 100 \%\end{array}$ & $\begin{array}{l}\text { A. Supervised group } \\
\text { class that includes } \\
\text { warm-up/ endurance, } \\
\text { jumping and resist- } \\
\text { ance exercise + home } \\
\text { training that includes } \\
\text { rope skipping, isomet- } \\
\text { ric exercises, elastic } \\
\text { belt and stretching } \\
\text { exercises } \\
\text { Frequency: Supervised } \\
\text { group classes: } 2 \text { times/ } \\
\text { week; home training } 2 \\
\text { times/week } \\
\text { (supervised group } \\
\text { classes: } 3 \text { times/week; } \\
\text { home training } 1 \text { time/ } \\
\text { week in the year } 4 \\
\text { and 5) } \\
\text { Intensity: } \\
\text { Aerobic dance: } 70 \% \text { to } \\
85 \% \text { maximum heart } \\
\text { rate and peak ground } \\
\text { reaction forces (GRF) } \\
\text { at approximately } 3 \text { to } \\
4 \text { times bodyweight; } \\
\text { Multilateral jumping: } 4 \\
\text { sets of } 15 \text { reps and } \\
\text { GRF at approximately } \\
4 \text { times of }\end{array}$ & $\begin{array}{l}\text { B. No training: } \\
\text { maintain own's } \\
\text { habitual lifestyle }\end{array}$ & $\begin{array}{l}\text { 1. Lumbar spine } \\
(\mathrm{L} 1-\mathrm{L} 4) \mathrm{BMD} \\
\text { 2. Femoral neck } \\
\text { BMD }\end{array}$ & 144 & $\begin{array}{l}\text { Mean difference } \\
(95 \% \mathrm{Cl}) \\
\text { 1. Lumbar spine } \\
\text { BMD } \\
0.030(0.011 \text { to } \\
0.049)^{\mathrm{c}} \\
\text { 2. Femoral neck } \\
\text { BMD } \\
0.024(0.009 \text { to } \\
0.039)^{\mathrm{c}}\end{array}$ \\
\hline
\end{tabular}


Table 1 Description of included studies comparing physical activity with a control intervention (Continued)

\begin{tabular}{|c|c|c|c|c|c|c|c|}
\hline $\begin{array}{l}\text { Reference } \\
\text { PEDro score }\end{array}$ & $\begin{array}{l}\text { Study } \\
\text { design } \\
\text { Allocated/ } \\
\text { Analysed }\end{array}$ & $\begin{array}{l}\text { Participants (n, } \\
\text { age mean (SD), \% } \\
\text { women, setting, } \\
\text { health status) }\end{array}$ & $\begin{array}{l}\text { Intervention } \\
\text { Primary exercise } \\
\text { type according to } \\
\text { ProFANE }^{\mathrm{a}}\end{array}$ & Control $^{b}$ & Outcomes & $\begin{array}{l}\text { Follow } \\
\text { up } \\
(\mathrm{mo})\end{array}$ & Results \\
\hline & & & $\begin{array}{l}\text { bodyweight; } \\
\text { Resistance: from } 1 \text { to } 4 \\
\text { sets, } 4 \text { to } 12 \text { reps, } 70 \% \\
\text { to } 90 \% 1 \text { RM (2 to } 3 \\
\text { minute-rest) to } 2 \text { to } 3 \\
\text { sets, } 20 \text { to } 25 \text { reps, } \\
50 \% \text { to } 55 \% 1 \text { RM (1 } \\
\text { to 2-minute rest) } \\
\text { Session duration: } 60 \\
\text { to } 65 \text { min/ supervised } \\
\text { group session; } \\
20 \text { min/home training } \\
\text { session } \\
\text { Delivered by: } \\
\text { Certified trainers } \\
\text { Duration of } \\
\text { intervention (wks): } \\
49 \text { to } 50 \text { weeks/year } \\
\text { throughout the } 12 \\
\text { years } \\
\text { Primary exercise } \\
\text { type: Multiple } \\
\text { (endurance plus } \\
\text { resistance with bone } \\
\text { loading) }\end{array}$ & & & & \\
\hline $\begin{array}{l}{ }^{d} \text { Kemmler } \\
2016 \S \\
4 / 10[93]\end{array}$ & $\begin{array}{l}\text { Quasi- } \\
\text { randomised } \\
\text { trial } \\
137 / 67\end{array}$ & $\begin{array}{l}\text { Setting: } \\
\text { Community; } \\
\text { Germany } \\
\text { Health status: } \\
\text { Osteopenia } \\
\text { A. Exercise group } \\
\boldsymbol{n}=86 \\
\text { (randomised); } 39 \\
\text { (analysed) } \\
\text { Age: } 55.0 \text { (3.5) } \\
\text { B. Control-no } \\
\text { training } \\
\boldsymbol{n}=51 \\
\text { (randomised); } 28 \\
\text { (analysed) } \\
\text { Age: } 56.0 \text { (3.0) } \\
\text { Female: } 100 \%\end{array}$ & $\begin{array}{l}\text { A. Supervised group } \\
\text { class (aerobic dance } \\
\text { exercise, jumping and } \\
\text { resistance exercise) + } \\
\text { Home training (rope } \\
\text { skipping, isometric } \\
\text { and dynamic } \\
\text { resistance exercise and } \\
\text { stretching/ flexibility } \\
\text { exercise) five months } \\
\text { after study started } \\
\text { Frequency: } \\
\text { Year } 4 \text { and } 5 \text { : } \\
\text { supervised group } \\
\text { classes: } 3 \text { times/week; } \\
\text { home training } 1 \text { time/ } \\
\text { week } \\
\text { All other years: } \\
\text { supervised group } \\
\text { classes: } 2 \text { times/week; } \\
\text { home training } 2 \\
\text { times/week } \\
\text { Intensity: } \\
\text { Aerobic dance: } 70 \% \text { to } \\
85 \% \text { maximum heart } \\
\text { rate and } 2 \text { to } 3 \\
\text { bodyweight peak } \\
\text { ground reaction forces } \\
\text { (GRF) } \\
\text { Multilateral jumping: } 4 \\
\text { sets of } 15 \text { reps at GRF } \\
\text { of } 3 \text { to } 4.5 \\
\text { bodyweightResistance } \\
\text { exercise: from } 1 \text { to } 4 \\
\text { sets of } 4 \text { to } 12 \text { reps at } \\
\text { intensity of } 70 \% \text { to } \\
90 \% 1 \text { RM (2- to } 3- \\
\text { minute rest) to } 2 \text { to } 3 \\
\text { sets of } 20 \text { to } 25 \text { reps } \\
\text { at an intensity of } 50 \% \\
\text { and }\end{array}$ & $\begin{array}{l}\text { B. No training - } \\
\text { maintain present } \\
\text { lifestyle }\end{array}$ & $\begin{array}{l}\text { 1. Lumbar spine } \\
\text { BMD } \\
\text { 2. Total hip BMD }\end{array}$ & 192 & 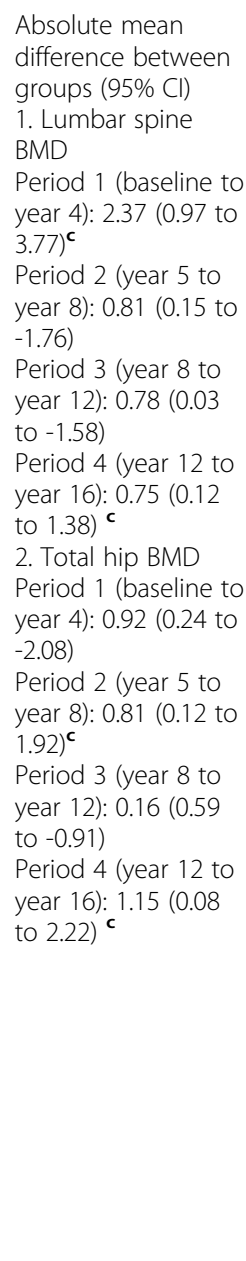 \\
\hline
\end{tabular}


Table 1 Description of included studies comparing physical activity with a control intervention (Continued)

\begin{tabular}{|c|c|c|c|c|c|c|c|}
\hline $\begin{array}{l}\text { Reference } \\
\text { PEDro score }\end{array}$ & $\begin{array}{l}\text { Study } \\
\text { design } \\
\text { Allocated/ } \\
\text { Analysed }\end{array}$ & $\begin{array}{l}\text { Participants (n, } \\
\text { age mean (SD), \% } \\
\text { women, setting, } \\
\text { health status) }\end{array}$ & $\begin{array}{l}\text { Intervention } \\
\text { Primary exercise } \\
\text { type according to } \\
\text { ProFANE }^{\mathrm{a}}\end{array}$ & Control $^{b}$ & Outcomes & $\begin{array}{l}\text { Follow } \\
\text { up } \\
(\mathrm{mo})\end{array}$ & Results \\
\hline & & & $\begin{array}{l}\text { to } 55 \% 1 \text { RM (1- to 2- } \\
\text { minute rest) } \\
\text { Session duration: } 60 \\
\text { to } 65 \text { min/ supervised } \\
\text { group session; } 20 \text { to } \\
25 \text { min/home training } \\
\text { session } \\
\text { Delivered by: NR } \\
\text { Duration of } \\
\text { intervention (wks): } \\
49 \text { to } 50 \text { weeks/year } \\
\text { throughout the } 16 \\
\text { years } \\
\text { Primary exercise } \\
\text { type: Multiple } \\
\text { (endurance plus } \\
\text { resistance with bone } \\
\text { loading) }\end{array}$ & & & & \\
\hline $\begin{array}{l}{ }^{d} \operatorname{Kim} 2018 \\
6 / 10[95]\end{array}$ & $\begin{array}{l}\text { Pilot RCT } \\
51 / 41\end{array}$ & $\begin{array}{l}\text { Setting: } \\
\text { Outpatient } \\
\text { department of a } \\
\text { hospital; South } \\
\text { Korea } \\
\text { Health status: } \\
\text { Diagnosis of Stage } \\
\text { I to III prostate } \\
\text { cancer receiving } \\
\text { androgen } \\
\text { deprivation } \\
\text { therapy without } \\
\text { osteoporosis } \\
\text { A. Home-based } \\
\text { exercise interven- } \\
\text { tion for prevent- } \\
\text { ing osteoporosis } \\
\text { (HEPO) } \\
\text { intervention } \\
\boldsymbol{n}=26 \\
\text { (randomised); } 23 \\
\text { (analysed) } \\
\text { Age: } 70.5 \text { (5.0) } \\
\text { B. Control- } \\
\text { stretching exer- } \\
\text { cise (STR) } \\
\boldsymbol{n}=25 \\
\text { (randomised); } 18 \\
\text { (analysed) } \\
\text { Age: } 71.0 \text { (5.5) } \\
\text { Female: } 0 \%\end{array}$ & $\begin{array}{l}\text { A. Home-based exer- } \\
\text { cise (HEPO). A core } \\
\text { program (weight-bear- } \\
\text { ing exercise and resist- } \\
\text { ance exercise) + } \\
\text { optional program } \\
\text { (stabilization/ balance } \\
\text { exercise and circuit re- } \\
\text { sistive calisthenics). } \\
\text { Two } 30 \text {-minute educa- } \\
\text { tion sessions with a } \\
\text { workbook preceded } \\
\text { the start of the exer- } \\
\text { cise and ten } 15- \\
\text { minute sessions of } \\
\text { telephone counselling } \\
\text { Frequency: } 3 \text { to } 5 \\
\text { times/week } \\
\text { Intensity: The weight- } \\
\text { bearing goal involved } \\
\text { at least } 150 \text { minutes } \\
\text { per week of } \\
\text { moderate-intensity } \\
\text { work, starting at an in- } \\
\text { tensity of } 11 \text { to } 12 \text { on } \\
\text { the rate of perceived } \\
\text { exertion scale and in- } \\
\text { creasing for } 6 \text { months } \\
\text { to } 13 \text { to } 15 \text {. } \\
\text { The resistance exercise } \\
\text { protocol started at } \\
\text { free weight and } \\
\text { gradually increased to } \\
\text { loads of } 10 \% \text { of body } \\
\text { weight. } \\
\text { Session duration: } \\
\sim 40 \text { min } \\
\text { Delivered by: Exercise } \\
\text { physiologist } \\
\text { Duration of the } \\
\text { intervention (wks): } \\
24 \\
\text { Primary exercise } \\
\text { type: Resistance with } \\
\text { bone loading }\end{array}$ & $\begin{array}{l}\text { B. Whole body } \\
\text { stretching exercise } \\
\text { (STR) }\end{array}$ & $\begin{array}{l}\text { 1. Lumbar spine } \\
\text { (L1-L4) BMD } \\
\text { 2. Femoral neck } \\
\text { BMD } \\
\text { 3. Total hip BMD }\end{array}$ & 6 & $\begin{array}{l}\text { Change score (mean } \\
\pm \text { SD) } \\
\text { 1. Lumbar spine (L1- } \\
\text { L4) BMD } \\
\text { A. HEPO: }-0.027 \pm \\
0.007 \\
\text { B. STR: }-0.031 \pm \\
\text { 0.008 } \\
\text { 2. Femoral neck } \\
\text { BMD } \\
\text { A. HEPO: }-0.014 \pm \\
\text { 0.007 } \\
\text { B. STR: }-0.015 \pm \\
\text { 0.008 } \\
\text { 3. Total hip BMD } \\
\text { A. HEPO: }-0.008 \pm \\
\text { 0.006 } \\
\text { B. STR: }-0.011 \pm \\
\text { 0.006 }\end{array}$ \\
\hline
\end{tabular}


Table 1 Description of included studies comparing physical activity with a control intervention (Continued)

\begin{tabular}{|c|c|c|c|c|c|c|c|}
\hline $\begin{array}{l}\text { Reference } \\
\text { PEDro score }\end{array}$ & $\begin{array}{l}\text { Study } \\
\text { design } \\
\text { Allocated/ } \\
\text { Analysed }\end{array}$ & $\begin{array}{l}\text { Participants (n, } \\
\text { age mean (SD), \% } \\
\text { women, setting, } \\
\text { health status) }\end{array}$ & $\begin{array}{l}\text { Intervention } \\
\text { Primary exercise } \\
\text { type according to } \\
\text { ProFANE }^{\mathrm{a}}\end{array}$ & Control $^{b}$ & Outcomes & $\begin{array}{l}\text { Follow } \\
\text { up } \\
\text { (mo) }\end{array}$ & Results \\
\hline $\begin{array}{l}\text { Kohrt } 1997 \\
3 / 10 \text { [55] }\end{array}$ & $\begin{array}{l}\text { Quasi- } \\
\text { randomised } \\
\text { trial } \\
39 / 30\end{array}$ & $\begin{array}{l}\text { Setting: NR; } \\
\text { United States } \\
\text { Health Status: } \\
\text { Healthy } \\
\text { A. Ground } \\
\text { reaction forces } \\
\text { training } \\
\boldsymbol{n}=14 \\
\text { (randomised); } 12 \\
\text { (analysed) } \\
\text { Age: } 66.0 \text { (1.0) } \\
\text { B. Joint reaction } \\
\text { forces training } \\
\boldsymbol{n}=13 \\
\text { (randomised); } 9 \\
\text { (analysed) } \\
\text { Age: } 65.0 \text { (1.0) } \\
\text { C. Control } \\
\boldsymbol{n}=12 \\
\text { (randomised); } 9 \\
\text { (analysed) } \\
\text { Age: } 68.0 \text { (1.0) } \\
\text { Female: 100\% }\end{array}$ & $\begin{array}{l}\text { A. Ground reaction } \\
\text { forces training: } \\
\text { Individualised exercise } \\
\text { training focusing on } \\
\text { activities that involved } \\
\text { ground-reaction } \\
\text { forces, such as walk- } \\
\text { ing, jogging and/or } \\
\text { stair climbing. } \\
\text { Frequency: } 3 \text { to } 5 \\
\text { times/week Intensity: } \\
\text { 60-70\% to } 80-85 \% \\
\text { maximum heart rate } \\
\text { Session duration: } 30- \\
45 \text { minutes/day } \\
\text { Delivered by: NR } \\
\text { Duration of the } \\
\text { intervention (wks): } \\
36 \\
\text { Primary exercise } \\
\text { type: Multiple } \\
\text { (balance and function } \\
\text { plus endurance plus } \\
\text { flexibility) } \\
\text { B. Joint reaction } \\
\text { forces training: } \\
\text { Individualised exercise } \\
\text { training including } \\
\text { activities that involved } \\
\text { joint-reaction forces, } \\
\text { such as weightlifting } \\
\text { and rowing. } \\
\text { Frequency: } 3 \text { to } 5 \\
\text { sessions/week } \\
\text { Intensity: } \\
\text { Weightlifting: } 2-3 \text { sets } \\
\text { of 8-12 reps; Rowing: } \\
60-70 \% \text { to } 80-85 \% \text { of } \\
\text { maximum heart rate } \\
\text { Session duration: NR } \\
\text { for the total session } \\
\text { duration; however; } \\
\text { rowing took } 15 \text { to } 20 \\
\text { min } \\
\text { Delivered by: NR } \\
\text { Duration of the } \\
\text { intervention (wks): } \\
\text { 36 } \\
\text { Primary exercise } \\
\text { type: Multiple } \\
\text { (resistance plus } \\
\text { endurance plus } \\
\text { flexibility) }\end{array}$ & C. No exercise & $\begin{array}{l}\text { 1. Whole body } \\
\text { BMD } \\
\text { 2. Lumbar spine } \\
\text { L2-L4 BMD } \\
\text { 3. Femoral neck } \\
\text { BMD } \\
\text { 4. Trochanter BMD } \\
\text { 5. Ward's BMD } \\
\text { 6. Ultra-distal wrist } \\
\text { BMD } \\
\text { 7. One-third distal } \\
\text { wrist BMD }\end{array}$ & 12 & $\begin{array}{l}\text { Between-group } \\
\text { analysis relative to } \\
\text { control } \\
\text { 1. Whole body BMD } \\
\text { A. Ground reaction: } \\
p<0.05 \\
\text { B. Joint reaction: } p< \\
0.01 \\
\text { 2. Lumbar spine L2- } \\
\text { L4 BMD } \\
\text { A. Ground reaction: } \\
p<0.05 \\
\text { B. Joint reaction: } p< \\
\text { 0.01 } \\
\text { 3. Femoral neck } \\
\text { BMD } \\
\text { A. Ground reaction: } \\
p<0.01 \\
\text { B. Joint reaction: no } \\
\text { difference } \\
\text { 4. Trochanter BMD } \\
\text { A. Ground reaction: } \\
\text { no difference } \\
\text { B. Joint reaction: no } \\
\text { difference } \\
\text { 5. Ward's BMD } \\
\text { A. Ground reaction: } \\
p<0.01 \\
\text { B. Joint reaction: } p< \\
\text { 0.05 } \\
\text { 6. Ultra-distal wrist } \\
\text { BMD } \\
\text { A. Ground reaction: } \\
\text { no difference } \\
\text { B. Joint reaction: no } \\
\text { difference } \\
\text { 7. One-third distal } \\
\text { wrist BMD } \\
\text { A. Ground reaction: } \\
\text { no difference } \\
\text { B. Joint reaction: no } \\
\text { difference } \\
\text { Quantitative } \\
\text { estimates were not } \\
\text { reported (chance } \\
\text { scores are provided } \\
\text { in a graph) }\end{array}$ \\
\hline $\begin{array}{l}{ }^{d} \text { Korpelainen } \\
\text { 2010‡ } \\
\text { 7/10 [96] }\end{array}$ & $\begin{array}{l}\text { RCT } \\
160 / 100\end{array}$ & $\begin{array}{l}\text { Setting: } \\
\text { Community; } \\
\text { Finland } \\
\text { Health status: } \\
\text { Women with hip } \\
\text { and radius } \\
\text { osteopenia } \\
\text { A. Exercise group } \\
\boldsymbol{n}=84 \\
\text { (randomised); } 55 \\
\text { (analysed) } \\
\text { Age: } 72.7 \text { (1.1) }\end{array}$ & $\begin{array}{l}\text { A. Supervised balance, } \\
\text { leg strength, and } \\
\text { impact training and } \\
\text { home exercise } \\
\text { Frequency: } 1 \text { time/ } \\
\text { week of training } \\
\text { session; } 1 \text { time/day of } \\
\text { home exercise training } \\
\text { Intensity: NR } \\
\text { Session duration: } 60 \\
\text { min/ supervised } \\
\text { session, and } 20 \text { min/ }\end{array}$ & B. Control & $\begin{array}{l}\text { 1. Femoral neck } \\
\text { BMD } \\
\text { 2. Trochanter BMD } \\
\text { 3. Total proximal } \\
\text { femur BMD } \\
\text { 4. Femoral neck } \\
\text { BMC } \\
\text { 5. Trochanter BMC } \\
\text { 6. Total proximal } \\
\text { femur BMC }\end{array}$ & $\begin{array}{l}48,60 \\
72\end{array}$ & $\begin{array}{l}\text { Mean difference } \\
(95 \% \mathrm{Cl}) \\
\text { 1. Femoral neck } \\
\text { BMD } \\
\text { At } 4 \text { year: } 0.01(-0.02 \\
\text { to } 0.03) \\
\text { At } 5 \text { year: } 0.01(-0.03 \\
\text { to } 0.02) \\
\text { At } 6 \text { year: } 0.00(-0.02 \\
\text { to } 0.02) \\
\text { 2. Trochanter BMD } \\
\text { At } 4 \text { year: } 0.01(-0.02\end{array}$ \\
\hline
\end{tabular}


Table 1 Description of included studies comparing physical activity with a control intervention (Continued)

\begin{tabular}{|c|c|c|c|c|c|c|c|}
\hline $\begin{array}{l}\text { Reference } \\
\text { PEDro score }\end{array}$ & $\begin{array}{l}\text { Study } \\
\text { design } \\
\text { Allocated/ } \\
\text { Analysed }\end{array}$ & $\begin{array}{l}\text { Participants (n, } \\
\text { age mean (SD), \% } \\
\text { women, setting, } \\
\text { health status) }\end{array}$ & $\begin{array}{l}\text { Intervention } \\
\text { Primary exercise } \\
\text { type according to } \\
\text { ProFANE }^{\mathrm{a}}\end{array}$ & Control $^{b}$ & Outcomes & $\begin{array}{l}\text { Follow } \\
\text { up } \\
\text { (mo) }\end{array}$ & Results \\
\hline & & $\begin{array}{l}\text { B. Control group } \\
\boldsymbol{n}=76 \\
\text { (randomised); } 45 \\
\text { (analysed) } \\
\text { Age: } 72.6(1.2) \\
\text { Female: } 100 \%\end{array}$ & $\begin{array}{l}\text { home exercise } \\
\text { following program } \\
\text { Delivered by: Physical } \\
\text { therapist } \\
\text { Duration of the } \\
\text { intervention: } 24 \\
\text { weeks/year } \\
\text { Primary exercise } \\
\text { type: Multiple } \\
\text { (balance and function } \\
\text { plus resistance with } \\
\text { bone loading) }\end{array}$ & & & & $\begin{array}{l}\text { to 0.03) } \\
\text { At } 5 \text { year: } 0.01(-0.02 \\
\text { to 0.03) } \\
\text { At } 6 \text { year: } 0.01(-0.02 \\
\text { to 0.04) } \\
\text { 3. Total proximal } \\
\text { femur BMD } \\
\text { At } 4 \text { year: } 0.01(-0.01 \\
\text { to 0.04) } \\
\text { At } 5 \text { year: } 0.01(-0.02 \\
\text { to 0.03) } \\
\text { At } 6 \text { year: } 0.01(-0.01 \\
\text { to 0.04) } \\
\text { 4. Femoral neck } \\
\text { BMC } \\
\text { At } 4 \text { year: }-0.01(-0.14 \\
\text { to 0.11) } \\
\text { At } 5 \text { year: }-0.03(-0.16 \\
\text { to 0.09) } \\
\text { At } 6 \text { year: }-0.01(-0.13 \\
\text { to 0.11) } \\
\text { 5. Trochanter BMC } \\
\text { At } 4 \text { year: }-0.22(-0.87 \\
\text { to 0.23) } \\
\text { At } 5 \text { year: }-0.30(-0.51 \\
\text { to 0.60) } \\
\text { At } 6 \text { year: }-0.25(-0.78 \\
\text { to 0.33) } \\
\text { 6. Total proximal } \\
\text { femur BMC } \\
\text { At } 4 \text { year: } 0.01(-1.56 \\
\text { to 0.76) } \\
\text { At } 5 \text { year: } 0.01(-1.72 \\
\text { to } 0.74) \\
\text { At } 6 \text { year: } 0.01(-1.68 \\
\text { to 0.81) }\end{array}$ \\
\hline $\begin{array}{l}{ }^{d} \text { Korpelainen } \\
2006 \neq \\
6 / 10[79]\end{array}$ & $\begin{array}{l}\text { RCT } \\
160 / 136\end{array}$ & $\begin{array}{l}\text { Setting: } \\
\text { Community; } \\
\text { Finland } \\
\text { Health status: } \\
\text { Women with hip } \\
\text { and radius } \\
\text { osteopenia } \\
\text { A. Exercise group } \\
\boldsymbol{n}=84 \\
\text { (randomised); } \\
69 \text { (analysed) } \\
\text { Age: } 72.9(1.1 \text { ) } \\
\text { B. Control group } \\
\boldsymbol{n}=76 \\
\text { (randomised); } 67 \\
\text { (analysed) } \\
\text { Age: } 72.8 \text { (1.2) } \\
\text { Female: } 100 \%\end{array}$ & $\begin{array}{l}\text { A. Supervised balance, } \\
\text { jumping, and impact } \\
\text { group training } \\
\text { Frequency: } 1 \text { time/ } \\
\text { week of training } \\
\text { session; } 1 \text { time/day of } \\
\text { home exercise training } \\
\text { Intensity: NR } \\
\text { Session duration: } 60 \\
\text { min/training session, } \\
\text { and } 20 \text { min/home } \\
\text { training } \\
\text { Delivered by: Physical } \\
\text { therapist } \\
\text { Duration of the } \\
\text { intervention (wks): } \\
24 \text { weeks/year } \\
\text { [exercise took place at } \\
\text { home for other times } \\
\text { during the year and in } \\
\text { total there were } 72 \\
\text { weeks supervised } \\
\text { group exercise] for } 30 \\
\text { months. } \\
\text { Primary exercise } \\
\text { type: Multiple } \\
\text { (balance and function } \\
\text { plus resistance with } \\
\text { bone loading) }\end{array}$ & B. Control & $\begin{array}{l}\text { 1. Femoral neck } \\
\text { BMD } \\
\text { 2. Trochanter BMD } \\
\text { 3. Total proximal } \\
\text { femur BMD } \\
\text { 4. Femoral neck } \\
\text { BMC } \\
\text { 5. Trochanter BMC } \\
\text { 6. Total proximal } \\
\text { femur BMC } \\
\text { 7. Distal radius } \\
\text { BMD } \\
\text { 8. Ultradistal } \\
\text { radius BMD }\end{array}$ & 30 & $\begin{array}{l}\text { Mean difference } \\
(95 \% \mathrm{Cl}) \\
\text { 1. Femoral neck } \\
\text { BMD } \\
0.007(-0.010 \text { to } \\
\text { 0.024) } \\
\text { 2. Trochanter BMD } \\
\text { 0.011 (-0.014 to } \\
\text { 0.035) } \\
\text { 3. Total proximal } \\
\text { femur BMD } \\
\text { 0.004 (-0.021 to } \\
\text { 0.030) } \\
\text { 4. Femoral neck } \\
\text { BMC } \\
\text {-0.018 (-0.134 to } \\
\text { 0.100) } \\
\text { 5. Trochanter BMC } \\
\text { 0.043 (-0.514 to } \\
\text { 0.600) } \\
\text { 6. Total proximal } \\
\text { femur BMC } \\
\text {-0.332 (-1.433 to } \\
\text { 0.769) } \\
\text { 7. Distal radius BMD } \\
\text {-0.003 (-0.017 to } \\
\text { 0.011) } \\
\text { 8. Ultradistal radius } \\
\text { BMD }\end{array}$ \\
\hline
\end{tabular}


Table 1 Description of included studies comparing physical activity with a control intervention (Continued)

\begin{tabular}{|c|c|c|c|c|c|c|c|}
\hline $\begin{array}{l}\text { Reference } \\
\text { PEDro score }\end{array}$ & $\begin{array}{l}\text { Study } \\
\text { design } \\
\text { Allocated/ } \\
\text { Analysed }\end{array}$ & $\begin{array}{l}\text { Participants (n, } \\
\text { age mean (SD), \% } \\
\text { women, setting, } \\
\text { health status) }\end{array}$ & $\begin{array}{l}\text { Intervention } \\
\text { Primary exercise } \\
\text { type according to } \\
\text { ProFANE }^{\mathrm{a}}\end{array}$ & Control $^{\mathbf{b}}$ & Outcomes & $\begin{array}{l}\text { Follow } \\
\text { up } \\
\text { (mo) }\end{array}$ & Results \\
\hline & & & & & & & $\begin{array}{l}-0.004(-0.018 \text { to } \\
0.008)\end{array}$ \\
\hline $\begin{array}{l}\text { Kwon } 2008 \\
3 / 10[56]\end{array}$ & $\begin{array}{l}\text { Quasi- } \\
\text { randomised } \\
\text { trial } \\
40 / \mathrm{NR}\end{array}$ & $\begin{array}{l}\text { Setting: } \\
\text { Community; Korea } \\
\text { Health status: } \\
\text { Healthy } \\
\text { A. } \\
\text { Multicomponent } \\
\text { intervention } \\
\boldsymbol{n}=20 \\
\text { (randomised) } \\
\text { Age: } 77.4 \text { (2.56) } \\
\text { B. Control } \\
\boldsymbol{n}=20 \\
\text { (randomised) } \\
\text { Age: } 77.0(3.33) \\
\text { Female: } 100 \%\end{array}$ & $\begin{array}{l}\text { A. Combined training } \\
\text { programme consisting } \\
\text { of aerobic exercise, } \\
\text { resistance training } \\
\text { (free weights) and } \\
\text { balance exercise. } \\
\text { Frequency: } 3 \text { times/ } \\
\text { week } \\
\text { Intensity: } \\
\text { Aerobic exercises: } \\
\text { started with } 40-55 \% \\
\text { and up to } 65-75 \% \\
\text { heart rate reserve; } \\
\text { Resistance exercise: } 8 \text { - } \\
12 \text { reps at } 75 \% \text { of } 1 \text { RM } \\
\text { Session duration: } 60 \\
\text { min } \\
\text { Delivered by: NR } \\
\text { Duration of the } \\
\text { intervention (wks): } \\
24 \\
\text { Primary exercise } \\
\text { type: Multiple } \\
\text { (balance and function } \\
\text { plus endurance plus } \\
\text { resistance) }\end{array}$ & B. Control & $\begin{array}{l}\text { 1. Whole body } \\
\text { BMD } \\
\text { 2. Lumbar (L2-L4) } \\
\text { spine BMD } \\
\text { 3. Femoral neck } \\
\text { BMD } \\
\text { 4. Ward's triangle } \\
\text { BMD } \\
\text { 5. Greater } \\
\text { trochanter BMD }\end{array}$ & 6 & $\begin{array}{l}\text { Final score (mean } \pm \\
\text { SD) } \\
\text { 1. Whole body BMD } \\
\text { A. Exercise: } 0.92 \pm \\
\text { 0.07 } \\
\text { B. Control: } 0.88 \pm \\
0.05 \\
\text { 2. Lumbar (L2-L4) } \\
\text { spine BMD } \\
\text { A. Exercise: } 0.85 \pm \\
0.15 \\
\text { B. Control: } 0.85 \pm \\
0.10 \\
\text { 3. Femoral neck } \\
\text { BMD } \\
\text { A. Exercise: } 0.68 \pm \\
0.12 \\
\text { B. Control: } 0.70 \pm \\
\text { 0.07 } \\
\text { 4. Ward's triangle } \\
\text { BMD } \\
\text { A. Exercise: } 0.48 \pm \\
\text { 0.10 } \\
\text { B. Control: } 0.46 \pm \\
\text { 0.08 } \\
\text { 5. Greater trochanter } \\
\text { BMD } \\
\text { A. Exercise: } 0.59 \pm \\
\text { 0.05 } \\
\text { B. Control: } 0.58 \pm \\
0.12\end{array}$ \\
\hline $\begin{array}{l}\text { Lau } 1992 \\
4 / 10[57]\end{array}$ & $\begin{array}{l}\text { RCT } \\
60 / 50\end{array}$ & $\begin{array}{l}\text { Setting: Hostel; } \\
\text { Hong Kong } \\
\text { Health Status: } \\
\text { Healthy } \\
\text { A. Exercise group } \\
\text { and placebo } \\
\text { calcium } \\
\text { supplementation } \\
\boldsymbol{n}=11 \text { (analysed) } \\
\text { Age: mean age } \\
\text { (range): } 79 \text { (76-81) } \\
\text { B. Calcium } \\
\text { supplementation } \\
\boldsymbol{n}=12 \text { (analysed) } \\
\text { Age: mean } \\
\text { age(range): } 75 \text { (72- } \\
79) \\
\text { C. Calcium } \\
\text { supplementation } \\
\text { and exercise } \\
\boldsymbol{n}=15 \text { (analysed) } \\
\text { Age: mean } \\
\text { age(range): } 76 \text { (73- } \\
\text { 80) } \\
\text { D. Control } \\
\boldsymbol{n}=12 \text { (analysed) } \\
\text { Age: mean age } \\
\text { (range): } 75 \text { years } \\
\text { (71-78) } \\
\text { Female: } 100 \%\end{array}$ & $\begin{array}{l}\text { A. Supervised exercise } \\
\text { involving moving the } \\
\text { upper trunk while } \\
\text { standing. } \\
\text { Frequency: } 4 \text { times/ } \\
\text { week } \\
\text { Intensity: Submaximal } \\
\text { exertion effort } \\
\text { Session duration: } 15 \\
\text { min } \\
\text { Delivered by: NR } \\
\text { Duration of the } \\
\text { intervention (wks): } \\
40 \\
\text { Primary exercise } \\
\text { type: Balance and } \\
\text { function }\end{array}$ & Control & $\begin{array}{l}\text { 1. Femoral neck } \\
\text { BMD } \\
\text { 2. Wards triangle } \\
\text { BMD } \\
\text { 3.Intertrochanteric } \\
\text { area BMD } \\
\text { 4. Lumbar spine } \\
\text { (L2-L4) BMD }\end{array}$ & 10 & $\begin{array}{l}\text { Change score (\%; } \\
\text { mean, } 95 \% \text { CI) } \\
\text { 1. Femoral neck } \\
\text { BMD } \\
\text { A. Exercise: -6.6 (-12 } \\
\text { to 0.8) } \\
\text { B. Control: -1.1 (-7.4 } \\
\text { to 5.3) } \\
\text { C. Supplement: -3.5 } \\
\text { (-9 to 1.8) } \\
\text { D. Supplement and } \\
\text { exercise: } 5.0 \text { (-0.77 to } \\
\text { 10) } \\
\text { 2. Wards triangle } \\
\text { BMD } \\
\text { A. Exercise: }-6.0 \text { (-15 } \\
\text { to 3.2) } \\
\text { B. Control: }-2.4 \text { (-10 } \\
\text { to 5.9) } \\
\text { C. Supplement: } 2.5 \\
\text { (-5.9 to } 11) \\
\text { D. Supplement and } \\
\text { exercise: } 17 \text { (3 to } 31) \\
\text { 3.Intertrochanteric } \\
\text { area BMD } \\
\text { A. Exercise: } 0.1 \text { (-6.5 } \\
\text { to } 6.7) \\
\text { B. Control: } 0.25 \text { (-3.3 } \\
\text { to 3.8) } \\
\text { C. Supplement: } 2 \\
\text { (-1.6 to } 5.7)\end{array}$ \\
\hline
\end{tabular}


Table 1 Description of included studies comparing physical activity with a control intervention (Continued)

\begin{tabular}{|c|c|c|c|c|c|c|c|}
\hline $\begin{array}{l}\text { Reference } \\
\text { PEDro score }\end{array}$ & $\begin{array}{l}\text { Study } \\
\text { design } \\
\text { Allocated/ } \\
\text { Analysed }\end{array}$ & $\begin{array}{l}\text { Participants ( } \mathrm{n} \text {, } \\
\text { age mean (SD), \% } \\
\text { women, setting, } \\
\text { health status) }\end{array}$ & $\begin{array}{l}\text { Intervention } \\
\text { Primary exercise } \\
\text { type according to } \\
\text { ProFANE }^{\mathrm{a}}\end{array}$ & Control $^{\mathbf{b}}$ & Outcomes & $\begin{array}{l}\text { Follow } \\
\text { up } \\
\text { (mo) }\end{array}$ & Results \\
\hline & & & & & & & $\begin{array}{l}\text { D. Supplement and } \\
\text { exercise: } 11 \text { (1.3 to } \\
\text { 22) } \\
\text { 4. Lumbar spine } \\
\text { BMD } \\
\text { A. Exercise: }-1.9 \text { (-6.7 } \\
\text { to 2.8) } \\
\text { B. Control: }-2.5 \text { (-6.5 } \\
\text { to 1.4) } \\
\text { C. Supplement: }-0.08 \\
\text { (-5.2 to 5.1) } \\
\text { D. Supplement and } \\
\text { exercise: }-1.1 \text { (-3.7 to } \\
\text { 1.4) }\end{array}$ \\
\hline $\begin{array}{l}\text { Lord } 1996 \\
4 / 10[58]\end{array}$ & $\begin{array}{l}\text { RCT } \\
179 / 138\end{array}$ & $\begin{array}{l}\text { Setting: } \\
\text { Community, } \\
\text { Australia } \\
\text { Health Status: } \\
\text { Healthy } \\
\text { A. } \\
\text { Multicomponent } \\
\text { exercise } \\
\boldsymbol{n}=90 \\
\text { (randomised); } 68 \\
\text { (analysed) } \\
\text { Age: } 71.7 \text { (5.4) } \\
\text { B. Control } \\
\boldsymbol{n}=89 \\
\text { (randomised); } 70 \\
\text { (analysed) } \\
\text { Age: } 71.5 \text { (5.3) } \\
\text { Female: } 100 \%\end{array}$ & $\begin{array}{l}\text { A. Supervised group- } \\
\text { based exercise } \\
\text { programme involving } \\
\text { aerobic exercise, bal- } \\
\text { ance training, } \\
\text { strengthening exercise, } \\
\text { and stretching. } \\
\text { Frequency: } 2 \text { times/ } \\
\text { week } \\
\text { Intensity: NR } \\
\text { Session duration: } 60 \\
\text { min } \\
\text { Delivered by: } \\
\text { Instructors trained to } \\
\text { provide the } \\
\text { programme } \\
\text { Duration of the } \\
\text { intervention (wks): } \\
52 \text { (only } 42 \text { weeks for } \\
\text { exercise as there were } \\
\text { breaks in between) } \\
\text { Primary exercise } \\
\text { type: Balance and } \\
\text { function }\end{array}$ & No exercise & $\begin{array}{l}\text { 1. Femoral neck } \\
\text { BMD } \\
\text { 2. Trochanter BMD } \\
\text { 3. Lumbar spine } \\
\text { (L2-L4) BMD }\end{array}$ & 12 & $\begin{array}{l}\text { Final score (mean } \pm \\
\text { SD) / Change score } \\
\text { (mean \% change } \pm \\
\text { SD) } \\
\text { 1. Femoral neck } \\
\text { BMD } \\
\text { A. Exercise: } 0.791 \pm \\
0.122 \text { / } 1.52 \pm 5.19 \\
\text { B. Control: } 0.776 \pm \\
0.110 / 3.12 \pm 6.52 \\
\text { 2. Trochanter BMD } \\
\text { A. Exercise: } 0.707 \pm \\
0.127 / 0.69 \pm 4,64 \\
\text { B. Control: } 0.672 \pm \\
\text { 0.123 / } 0.73 \pm 5.28 \\
\text { 3. Lumbar spine (L2- } \\
\text { L4) BMD } \\
\text { A. Exercise: } 1.036 \pm \\
\text { 0.209 / 1.07 } \pm 2.59 \\
\text { B. Control: } 1.008 \pm \\
\text { 0.189 / } 0.36 \pm 3.91\end{array}$ \\
\hline $\begin{array}{l}\text { Marques } \\
2011 \\
5 / 10[59]\end{array}$ & $\begin{array}{l}\text { RCT } \\
60 / 60\end{array}$ & $\begin{array}{l}\text { Setting: } \\
\text { Community; } \\
\text { Portugal } \\
\text { Health Status: } \\
\text { Healthy } \\
\text { A. Multi- } \\
\text { component } \\
\text { training } \\
\boldsymbol{n}=30 \text { (randomised } \\
\text { and analysed) } \\
\text { Age: } 70.1 \text { (5.4) } \\
\text { B. Control } \\
\boldsymbol{n}=30 \text { (randomised } \\
\text { and analysed) } \\
\text { Age: } 68.2 \text { (5.7) } \\
\text { Female: } 100 \%\end{array}$ & $\begin{array}{l}\text { A. Progressive } \\
\text { multicomponent } \\
\text { exercise training } \\
\text { consisting of } \\
\text { moderate to high } \\
\text { impact weight-bearing } \\
\text { activities, endurance, } \\
\text { balance exercise, and } \\
\text { agility training. } \\
\text { Frequency: } 2 \text { times/ } \\
\text { week } \\
\text { Intensity: Stepping } \\
\text { exercise: at } 120-125 \\
\text { beats/min. } \\
\text { Weight bearing and } \\
\text { strength exercise: from } \\
2 \text { sets of } 8 \text { reps to } 3 \\
\text { sets of } 15 \text { reps } \\
\text { Session duration: } 60 \\
\text { min } \\
\text { Delivered by: Physical } \\
\text { education instructors } \\
\text { specialised in physical } \\
\text { activity for older adults } \\
\text { Duration of the } \\
\text { intervention (wks): }\end{array}$ & B. Control & $\begin{array}{l}\text { 1. Femoral neck } \\
\text { BMD } \\
\text { 2. Total femur } \\
\text { BMD } \\
\text { 3. Trochanter BMD } \\
\text { 4. Intertrochanteric } \\
\text { BMD } \\
\text { 5. Lumbar spine } \\
\text { (L1-L4) BMD }\end{array}$ & 8 & $\begin{array}{l}\text { Final score (mean } \pm \\
\text { SD) } \\
\text { 1. Femoral neck } \\
\text { BMD } \\
\text { A. Exercise: } 0.717 \pm \\
0.085 \\
\text { B. Control: } 0.671 \pm \\
\text { 0.051 } \\
\text { 2. Total femur BMD } \\
\text { A. Exercise: } 0.832 \pm \\
\text { 0.104 } \\
\text { B. Control: } 0.823 \pm \\
\text { 0.058 } \\
\text { 3. Trochanter BMD } \\
\text { A. Exercise: } 0.628 \pm \\
\text { 0.081 } \\
\text { B. Control: } 0.628 \pm \\
\text { 0.034 } \\
\text { 4. Intertrochanteric } \\
\text { BMD } \\
\text { A. Exercise: } 0.989 \pm \\
\text { 0.148 } \\
\text { B. Control: } 0.977 \pm \\
\text { 0.075 } \\
\text { 5. Lumbar spine (L1- } \\
\text { L4) BMD }\end{array}$ \\
\hline
\end{tabular}


Table 1 Description of included studies comparing physical activity with a control intervention (Continued)

\begin{tabular}{|c|c|c|c|c|c|c|c|}
\hline $\begin{array}{l}\text { Reference } \\
\text { PEDro score }\end{array}$ & $\begin{array}{l}\text { Study } \\
\text { design } \\
\text { Allocated/ } \\
\text { Analysed }\end{array}$ & $\begin{array}{l}\text { Participants (n, } \\
\text { age mean (SD), \% } \\
\text { women, setting, } \\
\text { health status) }\end{array}$ & $\begin{array}{l}\text { Intervention } \\
\text { Primary exercise } \\
\text { type according to } \\
\text { ProFANE }^{\mathrm{a}}\end{array}$ & Control $^{\mathbf{b}}$ & Outcomes & $\begin{array}{l}\text { Follow } \\
\text { up } \\
(\mathrm{mo})\end{array}$ & Results \\
\hline & & & $\begin{array}{l}32 \\
\text { Primary exercise } \\
\text { type: Balance and } \\
\text { function with bone } \\
\text { loading (heel drops) }\end{array}$ & & & & $\begin{array}{l}\text { A. Exercise: } 0.868 \pm \\
0.094 \\
\text { B. Control: } 0.863 \pm \\
0.065\end{array}$ \\
\hline $\begin{array}{l}\text { McCartney } \\
1995 \\
3 / 10[60]\end{array}$ & $\begin{array}{l}\text { RCT } \\
68 / N R\end{array}$ & $\begin{array}{l}\text { Setting: NR; } \\
\text { Canada } \\
\text { Health status: } \\
\text { Healthy } \\
\text { A. Exercise } \\
\boldsymbol{n}=37 \\
\text { (randomised) } \\
\text { Age: } 73 \text { (3) } \\
\text { Female: } 54 \% \\
\text { B. Control } \\
\boldsymbol{n}=31 \text { (randomised) } \\
\text { Age: } 72 \text { (3) } \\
\text { Female: } 74 \%\end{array}$ & $\begin{array}{l}\text { A. Progressive } \\
\text { resistance training for } \\
\text { upper and lower body, } \\
\text { and abdominals. } \\
\text { Completed in as a } \\
\text { circuit. } \\
\text { Frequency: } 2 \\
\text { sessions/week } \\
\text { Intensity: } 2 \text { sets of } \\
\text { each exercise at } 50 \% \\
\text { of } 1 \text { RM to } 3 \text { sets of } \\
80 \% 1 R M \\
\text { Session duration: NR } \\
\text { Delivered by: NR } \\
\text { Duration of the } \\
\text { intervention (wks): } \\
42 \text { weeks } \\
\text { Primary exercise } \\
\text { type: Resistance }\end{array}$ & $\begin{array}{l}\text { B. Control } \\
\text { Offered a supervised } \\
\text { walking programme. } \\
\text { Frequency: } 2 \\
\text { sessions/week } \\
\text { Intensity: low } \\
\text { Session duration: } \\
\text { NR } \\
\text { Delivered by: NR } \\
\text { Duration of the } \\
\text { intervention (wks): } \\
42 \text { weeks }\end{array}$ & $\begin{array}{l}\text { 1. Lumbar spine } \\
\text { (L2-4) BMD } \\
\text { 2. Whole body } \\
\text { BMD } \\
\text { 3. Lumbar spine } \\
\text { (L2-4) BMC } \\
\text { 4. Whole body } \\
\text { BMC }\end{array}$ & 10.5 & $\begin{array}{l}\text { No significant } \\
\text { changes in BMD and } \\
\text { BMC as a result of } \\
\text { the training } \\
\text { programme. } \\
\text { Quantitative } \\
\text { estimates not } \\
\text { reported. }\end{array}$ \\
\hline $\begin{array}{l}\text { McMurdo } \\
1997 \\
4 / 10[61]\end{array}$ & $\begin{array}{l}\text { RCT } \\
118 / 92\end{array}$ & $\begin{array}{l}\text { Setting: } \\
\text { Community; } \\
\text { United Kingdom } \\
\text { Health status: } \\
\text { Healthy } \\
\text { A. Exercise and } \\
\text { calcium } \\
\text { supplementation } \\
\boldsymbol{n}=44 \text { (analysed) } \\
\text { B. Calcium } \\
\text { supplementation } \\
\boldsymbol{n}=48 \text { (analysed) } \\
\text { Age: } 64.5 \text { (range } \\
\text { 60-73) } \\
\text { Female: } 100 \%\end{array}$ & $\begin{array}{l}\text { A. Exercise } \\
\text { programme involving } \\
\text { weight bearing } \\
\text { exercise to music and } \\
\text { calcium } \\
\text { supplementation } \\
\text { (1000 mg calcium } \\
\text { daily, as calcium } \\
\text { carbonate) } \\
\text { Frequency: } 3 \text { times/ } \\
\text { week } \\
\text { Intensity: NR } \\
\text { Session duration: } 45 \\
\text { min } \\
\text { Delivered by: NR } \\
\text { Duration of the } \\
\text { intervention (wks): } \\
30 \text { (three 10-week } \\
\text { terms) } \\
\text { Primary exercise } \\
\text { type: Balance and } \\
\text { function }\end{array}$ & $\begin{array}{l}\text { B. Taking calcium } \\
\text { supplementation } \\
\text { (1000 mg calcium } \\
\text { daily, as calcium } \\
\text { carbonate) }\end{array}$ & $\begin{array}{l}\text { 1. Lumbar BMD } \\
\text { 2. Distal forearm } \\
\text { (non-dominant) } \\
\text { BMC } \\
\text { 3. Ultra distal } \\
\text { forearm (non- } \\
\text { dominant) BMC }\end{array}$ & 24 & $\begin{array}{l}\text { Change score (mean } \\
\% \text { change } \pm 95 \% \mathrm{Cl}) \\
\text { 1. Lumbar BMD } \\
\text { A. Exercise and } \\
\text { calcium: }-0.91 \text { (-6.8 } \\
\text { to 5.0) } \\
\text { B. Calcium: -2.65 } \\
\text { (-5.7 to 0.4) } \\
\text { 2. Distal forearm } \\
\text { (non-dominant) BMC } \\
\text { A. Exercise and } \\
\text { calcium: }-2.18 \text { (-3.0 } \\
\text { to -1.4) } \\
\text { B. Calcium: }-1.38 \\
\text { (-2.2 to -0.6) } \\
\text { 3. Ultra distal } \\
\text { forearm BMC } \\
\text { A. Exercise and } \\
\text { calcium: } 1.14 \text { (-0.8 to } \\
\text { 3.1) } \\
\text { B. Calcium: }-2.6 \text { (-4.6 } \\
\text { to -0.6) }\end{array}$ \\
\hline $\begin{array}{l}{ }^{e} \text { Nichols } \\
1995 \\
4 / 10 \text { [99] }\end{array}$ & $\begin{array}{l}\mathrm{RCT} \\
34 / 28\end{array}$ & $\begin{array}{l}\text { Setting: } \\
\text { Community; } \\
\text { United States } \\
\text { Health status: } \\
\text { Healthy and active } \\
\text { A. Weight } \\
\text { training group } \\
\boldsymbol{n}= \\
17 \text { (randomised); } 9 \\
\text { (analysed at 12- } \\
\text { month) } \\
\text { Age: 67.8 } \\
\text { (standard error: 1.6) } \\
\text { B. Control } \\
\boldsymbol{n}= \\
\text { 17(randomised); } 8 \\
\text { (analysed at 12- } \\
\text { month) }\end{array}$ & $\begin{array}{l}\text { A. Supervised, isotonic } \\
\text { training (leg flexion } \\
\text { and extension, back } \\
\text { extension, trunk } \\
\text { flexion, bench press, } \\
\text { latissimus dorsi pull- } \\
\text { down, shoulder press } \\
\text { and seated row) } \\
\text { Frequency: } 3 \text { times/ } \\
\text { week } \\
\text { Intensity: } \\
\text { Commenced with one } \\
\text { set of } 10-12 \text { reps at an } \\
\text { intensity of } 50 \% \text { of } \\
1 \text { RM and progressed } \\
\text { to three sets at } 80 \% \text { of } \\
\text { 1RM from third week } \\
\text { Session duration: NR }\end{array}$ & $\begin{array}{l}\text { B. Contunue current } \\
\text { endurance exercise } \\
\text { program }\end{array}$ & $\begin{array}{l}\text { 1. Lumbar spine } \\
(\text { L2-4) BMD2. } \\
\text { Femoral neck } \\
\text { BMD } \\
\text { 3. Trochanter BMD } \\
\text { 4. Total body BMD }\end{array}$ & 12 & $\begin{array}{l}\text { Final score (mean } \pm \\
\text { SE) } \\
\text { 1. Lumnar spine } \\
\text { BMD } \\
\text { A. Weight training: } \\
1.025 \pm 0.04 \\
\text { B. Control: } 1.012 \pm \\
0.03 \\
\text { 2. Femoral neck } \\
\text { BMD } \\
\text { A. Weight training: } \\
0.776 \pm 0.03 \\
\text { B. Control: } 0.772 \pm \\
0.02 \\
\text { 3. Trochanter BMD } \\
\text { A. Weight training: } \\
\text { 0.670 } \pm 0.02 \\
\text { B. Control: } 0.666 \pm\end{array}$ \\
\hline
\end{tabular}


Table 1 Description of included studies comparing physical activity with a control intervention (Continued)

\begin{tabular}{|c|c|c|c|c|c|c|c|}
\hline $\begin{array}{l}\text { Reference } \\
\text { PEDro score }\end{array}$ & $\begin{array}{l}\text { Study } \\
\text { design } \\
\text { Allocated/ } \\
\text { Analysed }\end{array}$ & $\begin{array}{l}\text { Participants (n, } \\
\text { age mean (SD), \% } \\
\text { women, setting, } \\
\text { health status) }\end{array}$ & $\begin{array}{l}\text { Intervention } \\
\text { Primary exercise } \\
\text { type according to } \\
\text { ProFANE }^{\mathrm{a}}\end{array}$ & Control $^{b}$ & Outcomes & $\begin{array}{l}\text { Follow } \\
\text { up } \\
(\mathrm{mo})\end{array}$ & Results \\
\hline & & $\begin{array}{l}\text { Age: } 65.2(1.2) \\
\text { Female: } 100 \%\end{array}$ & $\begin{array}{l}\text { Delivered by: NR } \\
\text { Duration of the } \\
\text { intervention (wks): } \\
52 \\
\text { Primary exercise } \\
\text { type: Resistance }\end{array}$ & & & & $\begin{array}{l}0.03 \\
\text { 4. Total body BMD } \\
\text { A. Weight training: } \\
0.976 \pm 0.02 \\
\text { B. Control: } 0.979 \pm \\
0.03\end{array}$ \\
\hline $\begin{array}{l}\text { Paillard } 2004 \\
5 / 10[62]\end{array}$ & $\begin{array}{l}\text { RCT } \\
21 / 21\end{array}$ & $\begin{array}{l}\text { Setting: } \\
\text { Community; } \\
\text { France } \\
\text { Health status: } \\
\text { Healthy } \\
\text { A. Walking group } \\
\boldsymbol{n}=11 \text { (randomised } \\
\text { and analysed) } \\
\text { Age: } 65.5 \text { (2) } \\
\text { B. Control } \\
\boldsymbol{n}=10 \text { (randomised } \\
\text { and analysed) } \\
\text { Age: } 66.8(2) \\
\text { Female: } 0 \%\end{array}$ & $\begin{array}{l}\text { A. Individualised brisk } \\
\text { walking programme } \\
\text { Frequency: } 5 \text { times/ } \\
\text { week } \\
\text { Intensity: Lactate } \\
\text { threshold (minimum } \\
\text { heart rate: } 131 \text { beats/ } \\
\text { minute; maximum } \\
\text { heart rate: } 156 \text { beats/ } \\
\text { minute) } \\
\text { Session duration: } 45 \\
\text { to } 60 \text { min } \\
\text { Delivered by: NR } \\
\text { Duration of the } \\
\text { intervention (wks): } \\
12 \\
\text { Primary exercise } \\
\text { type: Endurance } \\
\text { (walking) }\end{array}$ & B. Control & $\begin{array}{l}\text { 1. Hip BMD } \\
\text { 2. Whole body } \\
\text { BMD }\end{array}$ & 3 & $\begin{array}{l}\text { Final score (mean } \pm \\
\text { SD) } \\
\text { 1. Hip BMD } \\
\text { A. Walking: } 0.84 \pm \\
0.11 \\
\text { B. Control: } 0.95 \pm \\
0.12 \\
\text { 2. Whole body BMD } \\
\text { A. Walking: } 1.06 \pm \\
0.11 \\
\text { B. Control: } 1.02 \pm \\
0.13\end{array}$ \\
\hline $\begin{array}{l}\text { Park 2008 } \\
5 / 10[63]\end{array}$ & $\begin{array}{l}\text { RCT } \\
50 / 50\end{array}$ & $\begin{array}{l}\text { Setting: } \\
\text { Community; Korea } \\
\text { Health status: } \\
\text { Healthy } \\
\text { A. Multi- } \\
\text { component } \\
\text { training } \\
\boldsymbol{n}=25 \text { (randomised } \\
\text { and analysed) } \\
\text { Age: } 68.3 \text { (3.6) } \\
\text { B. Control } \\
\boldsymbol{n}=25 \text { (randomised } \\
\text { and analysed) } \\
\text { Age: } 68.4(3.4) \\
\text { Female: } 100 \%\end{array}$ & $\begin{array}{l}\text { A. Exercise training } \\
\text { including stretching, } \\
\text { strength training, } \\
\text { weight-bearing exer- } \\
\text { cise, balance and pos- } \\
\text { ture correction } \\
\text { training. } \\
\text { Frequency: } 3 \text { times/ } \\
\text { week } \\
\text { Intensity: } 65 \%-75 \% \text { of } \\
\text { the maximum heart } \\
\text { rate } \\
\text { Session duration: } 60 \\
\text { min } \\
\text { Delivered by: NR } \\
\text { Duration of the } \\
\text { intervention (wks): } \\
48 \\
\text { Primary exercise } \\
\text { type: Multiple } \\
\text { balance/ function plus } \\
\text { endurance (weight- } \\
\text { bearing) }\end{array}$ & B. Control & $\begin{array}{l}\text { 1. Femoral neck } \\
\text { BMD } \\
\text { 2. Ward's triangle } \\
\text { BMD } \\
\text { 3. Trochanter BMD } \\
\text { 4. Lumbar spine } \\
\text { (L2 to L4) BMD }\end{array}$ & 12 & $\begin{array}{l}\text { Final score (mean } \pm \\
\text { SD) } \\
\text { 1. Femoral neck } \\
\text { BMD } \\
\text { A. Exercise: } 0.857 \pm \\
0.078 \\
\text { B. Control: } 0.748 \pm \\
\text { 0.063 } \\
\text { 2. Ward's triangle } \\
\text { BMD } \\
\text { A. Exercise: } 0.659 \pm \\
\text { 0.086 } \\
\text { B. Control: } 0.576 \pm \\
\text { 0.079 } \\
\text { 3. Trochanter BMDc } \\
\text { A. Exercise: } 0.725 \pm \\
\text { 0.081 } \\
\text { B. Control: } 0.677 \pm \\
\text { 0.062 } \\
\text { 4. Lumbar spine (L2 } \\
\text { to L4) BMD } \\
\text { A. Exercise: } 1.059 \pm \\
\text { 0.082 } \\
\text { B. Control: } 0.891 \pm \\
\text { 0.155 }\end{array}$ \\
\hline $\begin{array}{l}\text { Pruitt } 1995 \\
4 / 10 \text { [64] }\end{array}$ & $\begin{array}{l}\text { RCT } \\
40 / 26\end{array}$ & $\begin{array}{l}\text { Setting: } \\
\text { Community; } \\
\text { America } \\
\text { Healthy status: } \\
\text { Healthy } \\
\text { A. High intensity } \\
\text { resistance } \\
\text { training } \\
\boldsymbol{n}=15 \\
\text { (randomised); } 8 \\
\text { (analysed) } \\
\text { Age: } 67.0 \text { (0.5) } \\
\text { B. Low intensity } \\
\text { resistance }\end{array}$ & $\begin{array}{l}\text { A. High intensity } \\
\text { resistance training: } \\
\text { High intensity } \\
\text { supervised resistance } \\
\text { training comprising } \\
\text { exercises for upper } \\
\text { and lower extremities } \\
\text { with the use of } \\
\text { equipment. } \\
\text { Intensity: } 2 \text { sets of } 7 \\
\text { reps at } 80 \% 1 R M \\
\text { Primary exercise } \\
\text { type: Resistance } \\
\text { B. Low intensity }\end{array}$ & C. No training & $\begin{array}{l}\text { 1. Total hip BMD } \\
\text { 2. Femoral neck } \\
\text { BMD } \\
\text { 3. Ward's triangle } \\
\text { BMD } \\
\text { 4. Lumbar spine } \\
\text { (L2-L4) BMD }\end{array}$ & 12 & $\begin{array}{l}\text { Change score (mean } \\
\pm \text { SD) } \\
\text { 1. Total hip BMD } \\
\text { A. High intensity: } \\
0.005 \pm 0.014 \\
\text { B. Low intensity: } \\
0.008 \pm 0.012 \\
\text { C. Control: } 0.007 \pm \\
0.010 \\
\text { 2. Femoral neck } \\
\text { BMD } \\
\text { A. High intensity: } \\
-0.002 \pm 0.154 \\
\text { B. Low intensity: }\end{array}$ \\
\hline
\end{tabular}


Table 1 Description of included studies comparing physical activity with a control intervention (Continued)

\begin{tabular}{|c|c|c|c|c|c|c|c|}
\hline $\begin{array}{l}\text { Reference } \\
\text { PEDro score }\end{array}$ & $\begin{array}{l}\text { Study } \\
\text { design } \\
\text { Allocated/ } \\
\text { Analysed }\end{array}$ & $\begin{array}{l}\text { Participants ( } n \text {, } \\
\text { age mean (SD), \% } \\
\text { women, setting, } \\
\text { health status) }\end{array}$ & $\begin{array}{l}\text { Intervention } \\
\text { Primary exercise } \\
\text { type according to } \\
\text { ProFANE }^{\mathrm{a}}\end{array}$ & Control $^{b}$ & Outcomes & $\begin{array}{l}\text { Follow } \\
\text { up } \\
\text { (mo) }\end{array}$ & Results \\
\hline & & $\begin{array}{l}\text { training } \\
\boldsymbol{n}=13 \\
\text { (randomised); } 7 \\
\text { (analysed) } \\
\text { Age: } 67.6(1.4) \\
\text { C. Control } \\
\boldsymbol{n}=12 \\
\text { (randomised); } 11 \\
\text { (analysed) } \\
\text { Age: } 69.6(4.2) \\
\text { Female: } 100 \%\end{array}$ & $\begin{array}{l}\text { resistance training: } \\
\text { Supervised resistance } \\
\text { training comprising } \\
\text { exercises for upper } \\
\text { and lower extremities } \\
\text { using equipment. } \\
\text { Intensity: } 3 \text { sets of } 14 \\
\text { reps at } 40 \% 1 R M \\
\text { For both A and B: } \\
\text { Frequency: } 3 \text { times/ } \\
\text { week } \\
\text { Session duration: } 50 \\
\text { to } 55 \text { min } \\
\text { Delivered by: NR } \\
\text { Duration of the } \\
\text { intervention (wks): } \\
52 \\
\text { Primary exercise } \\
\text { type: Resistance }\end{array}$ & & & & $\begin{array}{l}0.025 \pm 0.008 \\
\text { C. Control: } 0.005 \pm \\
0.019 \\
\text { 3. Ward's triangle } \\
\text { BMD } \\
\text { A. High intensity: } \\
0.018 \pm 0.032 \\
\text { B. Low intensity: } \\
0.022 \pm 0.045 \\
\text { C. Control: } 0.008 \pm \\
0.036 \\
\text { 4. Lumbar spine (L2- } \\
\text { L4) BMD } \\
\text { A. High intensity: } \\
0.007 \pm 0.018 \\
\text { B. Low intensity: } \\
\text { 0.005 } \pm 0.027 \\
\text { C. Control: } 0.000 \pm \\
0.020\end{array}$ \\
\hline $\begin{array}{l}\text { Rhodes } 2000 \\
5 / 10[65]\end{array}$ & $\begin{array}{l}\text { RCT } \\
44 / 38\end{array}$ & $\begin{array}{l}\text { Setting: } \\
\text { Community; } \\
\text { Canada } \\
\text { Health Status: } \\
\text { Healthy } \\
\text { A. Resistance } \\
\text { training } \\
\boldsymbol{n}=22 \\
\text { (randomised); } 20 \\
\text { (analysed) } \\
\text { Age: 68.8 (3.2) } \\
\text { B. Control } \\
\boldsymbol{n}=22 \\
\text { (randomised); } 18 \\
\text { (analysed) } \\
\text { Age: 68.2 (3.5) } \\
\text { Female: } 100 \%\end{array}$ & $\begin{array}{l}\text { A. Supervised } \\
\text { progressive resistance } \\
\text { training comprising } \\
\text { exercises for large } \\
\text { muscle groups. } \\
\text { Frequency: } 3 \text { times/ } \\
\text { week } \\
\text { Intensity: } 3 \text { sets of } 8 \\
\text { reps at } 75 \% 1 \text { RM } \\
\text { Session duration: } 60 \\
\text { min } \\
\text { Delivered by: } \\
\text { Professional lifestyle } \\
\text { and fitness consultants } \\
\text { Duration of the } \\
\text { intervention (wks): } \\
52 \\
\text { Primary exercise } \\
\text { type: Resistance }\end{array}$ & $\begin{array}{l}\text { B. No exercise } \\
\text { programme and } \\
\text { were instructed to } \\
\text { maintain their } \\
\text { normal lifestyle } \\
\text { throughout the } \\
\text { study duration. }\end{array}$ & $\begin{array}{l}\text { 1. Femoral neck } \\
\text { BMD } \\
\text { 2. Ward's triangle } \\
\text { BMD } \\
\text { 3. Trochanter BMD } \\
\text { 4. Lumbar spine } \\
\text { (L2-L4) BMD } \\
\text { 5. Femoral neck } \\
\text { BMC } \\
\text { 6. Ward's triangle } \\
\text { BMC } \\
\text { 7. Trochanter BMC } \\
\text { 8. Lumbar Spine } \\
\text { (L2-L4) BMC }\end{array}$ & 12 & 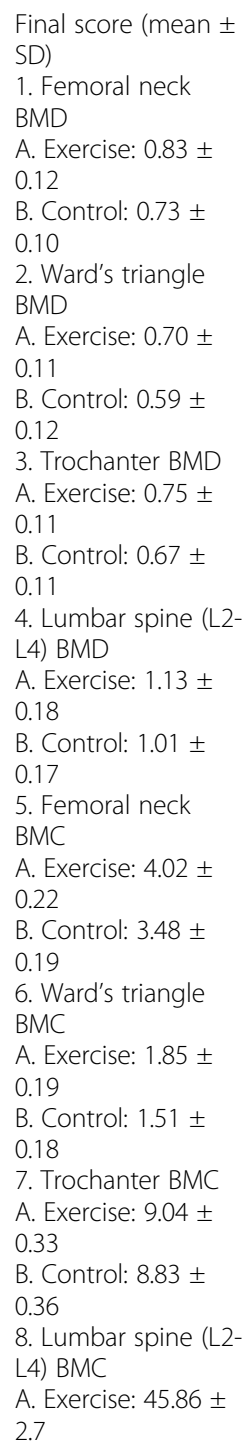 \\
\hline
\end{tabular}


Table 1 Description of included studies comparing physical activity with a control intervention (Continued)

\begin{tabular}{|c|c|c|c|c|c|c|c|}
\hline $\begin{array}{l}\text { Reference } \\
\text { PEDro score }\end{array}$ & $\begin{array}{l}\text { Study } \\
\text { design } \\
\text { Allocated/ } \\
\text { Analysed }\end{array}$ & $\begin{array}{l}\text { Participants (n, } \\
\text { age mean (SD), \% } \\
\text { women, setting, } \\
\text { health status) }\end{array}$ & $\begin{array}{l}\text { Intervention } \\
\text { Primary exercise } \\
\text { type according to } \\
\text { ProFANE }^{\mathrm{a}}\end{array}$ & Control $^{b}$ & Outcomes & $\begin{array}{l}\text { Follow } \\
\text { up } \\
\text { (mo) }\end{array}$ & Results \\
\hline & & & & & & & $\begin{array}{l}\text { B. Control: } 42.50 \pm \\
2.6\end{array}$ \\
\hline $\begin{array}{l}\text { Rikli } 1990 \\
1 / 10[67]\end{array}$ & $\begin{array}{l}\text { Quasi- } \\
\text { randomised } \\
\text { trial } \\
37 / 31\end{array}$ & $\begin{array}{l}\text { Setting: Local } \\
\text { retirement } \\
\text { community; United } \\
\text { States } \\
\text { Health Status: } \\
\text { Healthy } \\
\text { A. General } \\
\text { exercise } \\
\boldsymbol{n}=13 \\
\text { (randomised); } 10 \\
\text { (analysed) } \\
\text { Age: } 72.2 \text { (5.57) } \\
\text { B. General } \\
\text { exercise + weight } \\
\boldsymbol{n}=13 \\
\text { (randomised); } 10 \\
\text { (analysed) } \\
\text { Age: } 71.6 \text { (5.66) } \\
\text { C. Control } \\
\boldsymbol{n}=11 \\
\text { (randomised); } 11 \\
\text { (analysed) } \\
\text { Age: } 70.8 \text { (8.43) } \\
\text { Female: } 100 \%\end{array}$ & $\begin{array}{l}\text { A. General exercise: } \\
\text { Group-based aerobic } \\
\text { exercise training for } \\
\text { large muscle groups. } \\
\text { Frequency: } 3 \text { times/ } \\
\text { week } \\
\text { Intensity: } 60-70 \% \\
\text { maximum heart rate } \\
\text { Session duration: } 30 \\
\text { to } 50 \text { min } \\
\text { Delivered by: NR } \\
\text { Duration of the } \\
\text { intervention (wks): } \\
40 \\
\text { Primary exercise } \\
\text { type: endurance } \\
\text { B. General exercise + } \\
\text { weight: Group-based } \\
\text { aerobic exercise train- } \\
\text { ing plus upper body } \\
\text { progressive resistance } \\
\text { training. The resistance } \\
\text { training was per- } \\
\text { formed without } \\
\text { supervision. } \\
\text { Frequency: } 3 \text { times/ } \\
\text { week } \\
\text { Intensity: } 60-70 \% \\
\text { maximum heart rate } \\
\text { for aerobic activities } \\
\text { Session duration: } 50 \\
\text { to } 70 \text { min } \\
\text { Delivered by: } \\
\text { Assistants } \\
\text { Duration of the } \\
\text { intervention (wks): } \\
40 \\
\text { Primary exercise } \\
\text { type: Multiple } \\
\text { resistance plus } \\
\text { endurance }\end{array}$ & C. No exercise & $\begin{array}{l}\text { 1. Distal radius } \\
\text { BMC/BW } \\
\text { 2. Distal radius } \\
\text { BMC }\end{array}$ & 10 & $\begin{array}{l}\text { Change score (\%) } \\
\text { 1. Distal radius BMC/ } \\
\text { BWc } \\
\text { A. General exercise: } \\
0.921 \\
\text { B. General exercise } \\
\text { and weight: } 1.734 \\
\text { C. Control: -2.577 } \\
\text { 2. Distal radius BMC } \\
\text { A. General exercise: } \\
\text { 1.023 } \\
\text { B. General exercise } \\
\text { and weight: } 1.743 \\
\text { C. Control: }-2.499\end{array}$ \\
\hline $\begin{array}{l}\text { Sakai } 2010 \\
4 / 10[68]\end{array}$ & $\begin{array}{l}\text { RCT } \\
94 / 84\end{array}$ & $\begin{array}{l}\text { Setting: } \\
\text { Community, Japan } \\
\text { Health Status: } \\
\text { Healthy } \\
\text { A. Exercise } \\
\boldsymbol{n}=49 \\
\text { (randomised); } 47 \\
\text { (analysed) } \\
\text { Age: } 68.3 \text { (0.8) } \\
\text { B. Control } \\
\boldsymbol{n}=45 \\
\text { (randomised); } 37 \\
\text { (analysed) } \\
\text { Age: } 68.2(0.5) \\
\text { Female: } 100 \%\end{array}$ & $\begin{array}{l}\text { A. Home balance } \\
\text { exercises involving } \\
\text { unipedal standing } \\
\text { exercise with their } \\
\text { eyes open (single leg } \\
\text { standing) } \\
\text { Frequency: } 3 \text { sets/day; } \\
7 \text { days/week } \\
\text { Intensity: NA } \\
\text { Session duration: } 2 \\
\text { min/set } \\
\text { Delivered by: NA } \\
\text { (home exercise) } \\
\text { Duration of the } \\
\text { intervention (wks): } \\
26 \\
\text { Primary exercise } \\
\text { type: Balance/function }\end{array}$ & B. Usual activity & $\begin{array}{l}\text { 1. Femoral neck } \\
\text { BMD } \\
\text { 2. Trochanter BMD } \\
\text { 3. Intertrochanter } \\
\text { BMD } \\
\text { 4. Ward's triangle } \\
\text { BMD } \\
\text { 5. Total hip BMD }\end{array}$ & 6 & $\begin{array}{l}\text { \% mean difference } \\
\text { ( } \mathrm{p} \text {-value) } \\
\text { 1. Femoral neck: } p= \\
0.993 \\
\text { 2. Trochanter: } p= \\
0.801 \\
\text { 3. Intertrochanter: } p= \\
0.968 \\
\text { 4. Ward's triangle } p= \\
\text { 0.096 } \\
\text { 5. Total hip: } p=0.889 \\
\text { Change score } \\
\text { reported in a graph }\end{array}$ \\
\hline $\begin{array}{l}\text { Smith } 1981 \\
2 / 10[70]\end{array}$ & $\begin{array}{l}\text { Quasi- } \\
\text { randomised } \\
\text { trial }\end{array}$ & $\begin{array}{l}\text { Setting: Nursing } \\
\text { home; United } \\
\text { States }\end{array}$ & $\begin{array}{l}\text { A. Light-to-mild } \\
\text { seated exercises in- } \\
\text { cluding sideward leg }\end{array}$ & $\begin{array}{l}\text { B. Placebo tablets } \\
\text { Received } 360 \mathrm{mg} \text { of } \\
\text { lactose, } 5 \mathrm{mg} \text { of }\end{array}$ & 1. Radius BMC & 36 & $\begin{array}{l}\text { Change score (\%) } \\
\text { Radius BMC' } \\
\text { A. Exercise: } 2.29 \%\end{array}$ \\
\hline
\end{tabular}


Table 1 Description of included studies comparing physical activity with a control intervention (Continued)

\begin{tabular}{|c|c|c|c|c|c|c|c|}
\hline $\begin{array}{l}\text { Reference } \\
\text { PEDro score }\end{array}$ & $\begin{array}{l}\text { Study } \\
\text { design } \\
\text { Allocated/ } \\
\text { Analysed }\end{array}$ & $\begin{array}{l}\text { Participants ( } \mathrm{n} \text {, } \\
\text { age mean (SD), \% } \\
\text { women, setting, } \\
\text { health status) }\end{array}$ & $\begin{array}{l}\text { Intervention } \\
\text { Primary exercise } \\
\text { type according to } \\
\text { ProFANE }^{\mathrm{a}}\end{array}$ & Control $^{\mathbf{b}}$ & Outcomes & $\begin{array}{l}\text { Follow } \\
\text { up } \\
\text { (mo) }\end{array}$ & Results \\
\hline & $80 / 51$ & $\begin{array}{l}\text { Health Status: } \\
\text { Healthy } \\
\text { A. Physical } \\
\text { activity group + } \\
\text { placebo tablets } \\
\boldsymbol{n}=19 \\
\text { (randomised); } 12 \\
\text { (analysed) } \\
\text { Age: } 82.9 \text { (6.1) } \\
\text { B. Control } \\
\text { (placebo tablet) } \\
\boldsymbol{n}=26 \\
\text { (randomised); } 18 \\
\text { (analysed) } \\
\text { Age: } 81.9 \text { (7.4) } \\
\text { C. Calcium and } \\
\text { vitamin D } \\
\boldsymbol{n}=17 \\
\text { (randomised); } 10 \\
\text { (analysed) } \\
\text { Age: } 80.7 \text { (4.8) } \\
\text { D. Physical } \\
\text { activity + calcium } \\
\text { and vitamin D } \\
\boldsymbol{n}=18 \\
\text { (randomised); } 11 \\
\text { (analysed) } \\
\text { Age: } 84.3 \text { (5.1) } \\
\text { Female: } 100 \%\end{array}$ & $\begin{array}{l}\text { spread, leg walk, run- } \\
\text { ning in place, arm } \\
\text { cross, sideward bend } \\
\text { and chair pull. } \\
\text { Frequency: } 3 \text { times/ } \\
\text { week } \\
\text { Intensity: } 70 \% \text { of the } \\
\text { sampled } \mathrm{VO}_{2} \text { max } \\
\text { Session duration:30 } \\
\text { min } \\
\text { Delivered by: NR } \\
\text { Duration of the } \\
\text { intervention (wks): } \\
156 \\
\text { Primary exercise } \\
\text { type: Endurance } \\
\text { (seated) }\end{array}$ & $\begin{array}{l}\text { magnesium stearate } \\
\text { and } 80 \mathrm{mg} \text { of } \\
\text { microcrystalline } \\
\text { cellulose in the } \\
\text { placebo tablets }\end{array}$ & & & D. Control: - 3.29\% \\
\hline $\begin{array}{l}\text { eSnow } 2000 \\
3 / 10[100]\end{array}$ & $\begin{array}{l}\text { Quasi- } \\
\text { randomised } \\
\text { trial } \\
18 / 18\end{array}$ & $\begin{array}{l}\text { Setting: } \\
\text { Community; } \\
\text { United States } \\
\text { Health status: } \\
\text { Healthy } \\
\text { A. Exercise group } \\
\boldsymbol{n}=9 \text { (randomised } \\
\text { and analysed) } \\
\text { Age: } 66.4(1.7) \\
\text { B. Control } \\
\boldsymbol{n}=9 \text { (randomised } \\
\text { and analysed) } \\
\text { Age: } 61.8(2.5) \\
\text { Female:100\% }\end{array}$ & $\begin{array}{l}\text { A. Year1: 9-month } \\
\text { training (10 min of } \\
\text { warm-up, } 35 \text { min of } \\
\text { lower-body resistance } \\
\text { training, including } \\
\text { stepping, squats, chair } \\
\text { raises, forward lunges, } \\
\text { lateral lunges and toe } \\
\text { raises, using the } \\
\text { weighted vest, 10-15 } \\
\text { min of cool-down ac- } \\
\text { tivities. Jumping exer- } \\
\text { cises were included } \\
\text { during the fourth } \\
\text { months of training } \\
\text { without the weighted } \\
\text { vests } \\
\text { Year } 2-5 \text { emphasied } \\
\text { maintenance in lower } \\
\text { body exercises utilising } \\
\text { the weighted vest in- } \\
\text { cluded more jumps } \\
\text { per class than year } 1 \\
\text { and encouraged use } \\
\text { of the weighted vest } \\
\text { while jumping. } \\
\text { Frequency: } 3 \text { times/ } \\
\text { week } \\
\text { Intensity: } 3 \text { to } 5 \text { sets } \\
\text { of } 10 \text { to } 15 \text { reps using } \\
\text { the weight vest. } \\
\text { Vest resistance set } \\
\text { from } 5 \% \text { of body } \\
\text { weight and was } \\
\text { gradually increased } \\
\text { (approximaltey } 1 \text { to }\end{array}$ & $\begin{array}{l}\text { B. Maintain and } \\
\text { record their physical } \\
\text { activity during the } \\
\text { study and did not } \\
\text { engage in weighted } \\
\text { vest of jumping } \\
\text { activity }\end{array}$ & $\begin{array}{l}\text { 1. Femoral neck } \\
\text { BMD } \\
\text { 2. Trochanter BMD } \\
\text { 3. Total hip BMD }\end{array}$ & 60 & $\begin{array}{l}\text { \% change (mean } \pm \\
\text { SE) } \\
\text { 1. Femoral neck } \\
\text { BMDc } \\
\text { A. Exercise group: } \\
\text { 1.54 } \pm 2.37 \\
\text { B. Control: }-4.43 \pm \\
0.93 \\
\text { 2. Trochanter BMD } \\
\text { A. Exercise group: } \\
-0.24 \pm 1.02 \\
\text { B. Control: }-3.43 \% \pm \\
\text { 1.09 } \\
\text { 3. Total hip BMD } \\
\text { A. Exercise group: } \\
-0.82 \pm 1.04 \\
\text { B. Control: }-3.80 \% \pm \\
\text { 1.03\% }\end{array}$ \\
\hline
\end{tabular}


Table 1 Description of included studies comparing physical activity with a control intervention (Continued)

\begin{tabular}{|c|c|c|c|c|c|c|c|}
\hline $\begin{array}{l}\text { Reference } \\
\text { PEDro score }\end{array}$ & $\begin{array}{l}\text { Study } \\
\text { design } \\
\text { Allocated/ } \\
\text { Analysed }\end{array}$ & $\begin{array}{l}\text { Participants (n, } \\
\text { age mean (SD), \% } \\
\text { women, setting, } \\
\text { health status) }\end{array}$ & $\begin{array}{l}\text { Intervention } \\
\text { Primary exercise } \\
\text { type according to } \\
\text { ProFANE }^{\mathrm{a}}\end{array}$ & Control $^{\mathbf{b}}$ & Outcomes & $\begin{array}{l}\text { Follow } \\
\text { up } \\
\text { (mo) }\end{array}$ & Results \\
\hline & & & $\begin{array}{l}2 \% \text { every } 2 \text { weeks) } \\
\text { until } 10 \% \text { of body } \\
\text { weight; Beyond 10\% } \\
\text { of body weight, } \\
\text { resistance was } \\
\text { increased at } 0.5 \% \text { to } \\
1 \% \text { every } 2 \text { weeks. } \\
\text { Session duration: } 60 \\
\text { Delivered by: NR } \\
\text { Duration of the } \\
\text { intervention (wks): } \\
260 \\
\text { Primary exercise } \\
\text { type: Resistance with } \\
\text { bone loading } \\
\text { (jumping) }\end{array}$ & & & & \\
\hline $\begin{array}{l}\text { Taaffe } 1996 \\
4 / 10[101]\end{array}$ & $\begin{array}{l}\text { RCT } \\
36 / 21\end{array}$ & $\begin{array}{l}\text { Setting: } \\
\text { Community; } \\
\text { United States } \\
\text { Health status: } \\
\text { Healthy } \\
\text { A. High intensity } \\
\text { resistance } \\
\text { training group } \\
\boldsymbol{n}=12 \\
\text { (randomised); } 7 \\
\text { (analysed) } \\
\text { Age: } 67.0 \text { (0.2) } \\
\text { B. Low intensity } \\
\text { resistance } \\
\text { training group } \\
\boldsymbol{n}=13 \\
\text { (randomised); } 7 \\
\text { (analysed) } \\
\text { Age: } 67.6(0.5) \\
\text { C. Control } \\
\boldsymbol{n}=11 \\
\text { (randomised); } 7 \\
\text { (analysed) } \\
\text { Age: } 69.6 \text { (1.3) } \\
\text { Female: 100\% }\end{array}$ & $\begin{array}{l}\text { Supervised exercise } \\
\text { training targeted thigh } \\
\text { muscle strength } \\
\text { including leg press, } \\
\text { knee extension and } \\
\text { knee flexion. Exercise } \\
\text { sessions were } \\
\text { bracketed by warm up } \\
\text { and cool-down } \\
\text { periods } \\
\text { A. Intensity: } 1 \text { set of } \\
14 \text { reps at an intensity } \\
\text { of } 40 \% 1 \text { RM and } 2 \text { sets } \\
\text { of } 7 \text { reps at an } \\
\text { intensity of } 80 \% \text { of } \\
1 \text { RM } \\
\text { B. Intensity: } 3 \text { sets of } \\
14 \text { reps at an intensity } \\
\text { of } 40 \% \text { of } 1 \text { RM } \\
\text { For both groups } \\
\text { Frequency: } 3 \text { times/ } \\
\text { week } \\
\text { Session duration: NR } \\
\text { Delivered by: NR } \\
\text { Duration of the } \\
\text { intervention (wks): } \\
52 \\
\text { Primary exercise } \\
\text { type: Resistance }\end{array}$ & D. Control & $\begin{array}{l}\text { 1. Middle third of } \\
\text { the femur BMD } \\
\text { 2. Thigh BMD }\end{array}$ & 12 & $\begin{array}{l}\text { Change score (mean } \\
\% \pm \text { SEM) } \\
\text { 1. Middle third of } \\
\text { the femur BMD }{ }^{\mathrm{C}} \\
\text { A. High intensity: } \\
1.0 \pm 1.0 \\
\text { B. Low inteisty: }-2.2 \\
\pm 0.5 \\
\text { C. Control: }-1.8 \pm 0.6 \\
\text { 2. NR }\end{array}$ \\
\hline $\begin{array}{l}\text { Taaffe 1999 } \\
5 / 10 \text { [72] }\end{array}$ & $\begin{array}{l}\text { RCT } \\
53 / 46\end{array}$ & $\begin{array}{l}\text { Setting: } \\
\text { Community; } \\
\text { United States } \\
\text { Health status: } \\
\text { Healthy } \\
\text { A. High-intensity } \\
\text { resistance train- } \\
\text { ing (1 day per } \\
\text { week) } \\
\boldsymbol{n}=14 \\
\text { (randomised); } 11 \\
\text { (analysed) } \\
\text { Age: } 68.5 \text { (3.6) } \\
\text { Female: } 36 \% \\
\text { B. High-intensity } \\
\text { resistance train- } \\
\text { ing (2 days per } \\
\text { week) } \\
\boldsymbol{n}=14\end{array}$ & $\begin{array}{l}\text { A, B \& C. Supervised } \\
\text { resistance training } \\
\text { targeting the major } \\
\text { upper and lower body } \\
\text { muscle groups. } \\
\text { A. Frequency: } 1 \text { time/ } \\
\text { week } \\
\text { B. Frequency: } 2 \\
\text { times/week } \\
\text { C. Frequency: } 3 \\
\text { times/week } \\
\text { Intensity: Started at } \\
60 \% \text { of the } 1 \text { RM and } \\
\text { gradually increase in } \\
\text { intensity to } 3 \text { sets of } 8 \\
\text { reps at } 80 \% \text { of } 1 \text { RM } \\
\text { Session duration: NR } \\
\text { Delivered by: NR } \\
\text { Duration of the }\end{array}$ & D. Control & $\begin{array}{l}\text { 1. Lumbar spine } \\
\text { (L2-L4) BMD } \\
\text { 2. Total hip BMD } \\
\text { 3. Midradius BMD } \\
\text { 4. Total body BMC }\end{array}$ & 6 & $\begin{array}{l}\text { Final score (mean } \pm \\
\text { SEM) } \\
\text { 1. Lumbar spine (L2- } \\
\text { L4) BMD } \\
\text { A. Resistance } 1 \times / \\
\text { week: } 1.025 \pm 0.006 \\
\text { B. Resistance } 2 \mathrm{x} / \\
\text { week: } 1.033 \pm 0.006 \\
\text { C. Resistance } 3 \mathrm{x} / \\
\text { week: } 1.032 \pm 0.007 \\
\text { D. Control: } 1.041 \pm \\
\text { 0.006 } \\
\text { 2. Total hip BMD } \\
\text { A. Resistance } 1 \mathrm{x} / \\
\text { week: } 0.865 \pm 0.010 \\
\text { B. Resistance } 2 \mathrm{x/} \\
\text { week: } 0.866 \pm 0.006 \\
\text { C. Resistance } 3 \times / \\
\text { week: } 0.864 \pm 0.010\end{array}$ \\
\hline
\end{tabular}


Table 1 Description of included studies comparing physical activity with a control intervention (Continued)

\begin{tabular}{|c|c|c|c|c|c|c|c|}
\hline $\begin{array}{l}\text { Reference } \\
\text { PEDro score }\end{array}$ & $\begin{array}{l}\text { Study } \\
\text { design } \\
\text { Allocated/ } \\
\text { Analysed }\end{array}$ & $\begin{array}{l}\text { Participants (n, } \\
\text { age mean (SD), \% } \\
\text { women, setting, } \\
\text { health status) }\end{array}$ & $\begin{array}{l}\text { Intervention } \\
\text { Primary exercise } \\
\text { type according to } \\
\text { ProFANE }^{\mathrm{a}}\end{array}$ & Control $^{\mathbf{b}}$ & Outcomes & $\begin{array}{l}\text { Follow } \\
\text { up } \\
\text { (mo) }\end{array}$ & Results \\
\hline & & $\begin{array}{l}\text { (randomised); } 12 \\
\text { (analysed) } \\
\text { Age: } 69.4 \text { (3.0) } \\
\text { Female: } 29 \% \\
\text { C. High-intensity } \\
\text { resistance train- } \\
\text { ing (3 days per } \\
\text { week) } \\
\boldsymbol{n}=11 \text { (randomised } \\
\& \text { analysed) } \\
\text { Age: } 71.0 \text { (4.1) } \\
\text { Female: } 36 \% \\
\text { D. Control } \\
\boldsymbol{n}=14 \\
\text { (randomised); } 12 \\
\text { (analysed) } \\
\text { Age: } 68.9 \text { (3.6) } \\
\text { Female: } 43 \%\end{array}$ & $\begin{array}{l}\text { intervention (wks): } \\
24 \\
\text { Primary exercise } \\
\text { type: Resistance }\end{array}$ & & & & $\begin{array}{l}\text { D. Control: } 0.873 \pm \\
0.010 \\
\text { 3. Midradius BMD } \\
\text { A. Resistance } 1 \times / \\
\text { week: } 0.605 \pm 0.003 \\
\text { B. Resistance } 2 \text { x/ } \\
\text { week: } 0.604 \pm 0.003 \\
\text { C. Resistance } 3 \times / \\
\text { week: } 0.608 \pm 0.003 \\
\text { D. Control: } 0.601 \pm \\
\text { 0.003 } \\
\text { 4. Total body BMC } \\
\text { A. Resistance 1x/ } \\
\text { week: } 2552 \pm 15 \\
\text { B. Resistance } 2 \text { x/ } \\
\text { week: } 2530 \pm 14 \\
\text { C. Resistance } 3 \times / \\
\text { week: } 2525 \pm 14 \\
\text { D. Control: } 2536 \pm \\
\text { 14 }\end{array}$ \\
\hline $\begin{array}{l}\text { Villareal } 2003 \\
4 / 10 \text { [73] }\end{array}$ & $\begin{array}{l}\text { Quasi- } \\
\text { randomised } \\
\text { trial } \\
28 / 28\end{array}$ & $\begin{array}{l}\text { Setting: } \\
\text { Community; } \\
\text { United States } \\
\text { Health status: } \\
\text { Mild to moderate } \\
\text { physical frailty on } \\
\text { hormone } \\
\text { replacement } \\
\text { therapy } \\
\text { A. Supervised } \\
\text { multi-component } \\
\text { training } \\
\boldsymbol{n}=14 \text { (randomised } \\
\text { and analysed) } \\
\text { Age: } 81 \text { (3) } \\
\text { B. Control: Home } \\
\text { exercise } \\
\boldsymbol{n}=14 \text { (randomised } \\
\text { and analysed) } \\
\text { Age: } 81 \text { (3) } \\
\text { Female: } 100 \%\end{array}$ & $\begin{array}{l}\text { A. Supervised exercise } \\
\text { programme involving } \\
\text { flexibility and balance } \\
\text { exercises, resistance } \\
\text { training and } \\
\text { endurance exercises. } \\
\text { Frequency: } 3 \text { times/ } \\
\text { week } \\
\text { Intensity: } \\
\text { Resistance training: } \\
\text { from } 1-2 \text { sets of } 8-12 \\
\text { reps at } 65 \% \text { of } 1 \text { RM to } \\
2-3 \text { sets of } 6-8 \text { reps at } \\
75-85 \% \text { of } 1 \text { RM } \\
\text { Endurance: from } 65- \\
75 \text { to } 85-90 \% \text { peak } \\
\text { heart rate } \\
\text { Session duration: } 90 \\
\text { to } 120 \text { min } \\
\text { Delivered by: Exercise } \\
\text { physiologists } \\
\text { Duration of the } \\
\text { intervention (wks): } \\
36 \text { weeks } \\
\text { Primary exercise } \\
\text { type: Multiple } \\
\text { (resistance plus } \\
\text { balance/function plus } \\
\text { endurance) }\end{array}$ & $\begin{array}{l}\text { B. Home exercise } \\
\text { programme } \\
\text { focusing on } \\
\text { flexibility } \\
\text { Frequency: 2-3 } \\
\text { times/week }\end{array}$ & $\begin{array}{l}\text { 1. Total hip BMD } \\
\text { 2. Femoral neck } \\
\text { BMD } \\
\text { 3. Trochanter BMD } \\
\text { 4. Lumbar spine } \\
\text { BMD } \\
\text { 5. Whole body } \\
\text { BMD }\end{array}$ & 9 & $\begin{array}{l}\text { Change score (mean } \\
\pm \text { SD) } \\
\text { 1. Total hip BMD } \\
\text { A. Exercise: } 0.003 \pm \\
0.011 \\
\text { B. Control: } 0.009 \pm \\
0.011 \\
\text { 4. Lumbar spine } \\
\text { BMD } \\
\text { A. Exercise: } 0.034 \pm \\
0.022 \\
\text { B. Control: } 0.015 \pm \\
0.022 \\
\text { 5. Whole body BMD } \\
\text { A. Exercise: } 0.015 \pm \\
\text { 0.015 } \\
\text { B. Control: } 0.002 \pm \\
\text { 0.015 } \\
\text { No quantitative } \\
\text { estimates reported } \\
\text { for: } \\
\text { 2. Femoral neck } \\
\text { BMD } \\
\text { 3. Trochanter BMD } \\
\text { (There were no } \\
\text { significant group-by- } \\
\text { time interaction } \\
\text { effects) } \\
\text { Results reported in a } \\
\text { graph }\end{array}$ \\
\hline $\begin{array}{l}\text { Villareal } 2004 \\
4 / 10 \text { [74] }\end{array}$ & $\begin{array}{l}\text { RCT } \\
119 / 112\end{array}$ & $\begin{array}{l}\text { Setting: } \\
\text { Community; } \\
\text { United States } \\
\text { Health status: } \\
\text { Mild-to-moderate } \\
\text { physical frailty } \\
\text { A. Exercise } \\
\text { training (ET) } \\
\boldsymbol{n}=69 \\
\text { (randomised); } 65 \\
\text { (analysed) } \\
\text { Age: } 83 \text { (4) } \\
\text { Female: 52\% } \\
\text { B. Home exercise }\end{array}$ & $\begin{array}{l}\text { A. Supervised exercise } \\
\text { programme involving } \\
\text { flexibility and balance } \\
\text { exercises, resistance } \\
\text { training and } \\
\text { endurance exercises. } \\
\text { Frequency: NR } \\
\text { Intensity: } \\
\text { Resistance training: } \\
\text { started from } 1-2 \text { sets } \\
\text { of } 6-8 \text { reps at } 65-75 \% \\
\text { of } 1 \text { RM to } 3 \text { sets of } 8- \\
12 \text { reps at } 85-100 \% \\
1 \text { RM }\end{array}$ & $\begin{array}{l}\text { B. Home exercise } \\
\text { programme } \\
\text { focusing on } \\
\text { flexibility }\end{array}$ & $\begin{array}{l}\text { 1. Total hip BMD } \\
\text { 2. Femoral neck } \\
\text { BMD } \\
\text { 3. Trochanter BMD } \\
\text { 4. Lumbar spine } \\
\text { (L2-L4) BMD } \\
\text { 5. Whole Body } \\
\text { BMD }\end{array}$ & 9 & $\begin{array}{l}\text { Final score (mean } \pm \\
\text { SD) } \\
\text { 1. Total hip BMD } \\
\text { A. Exercise: } 0.85 \pm \\
0.19 \\
\text { B. Control: } 0.75 \pm \\
0.15 \\
\text { 2. Femoral neck } \\
\text { BMD } \\
\text { A. Exercise: } 0.70 \pm \\
\text { 0.17 } \\
\text { B. Control: } 0.63 \pm \\
\text { 0.11 } \\
\text { 3. Trochanter BMD }\end{array}$ \\
\hline
\end{tabular}


Table 1 Description of included studies comparing physical activity with a control intervention (Continued)

\begin{tabular}{|c|c|c|c|c|c|c|c|}
\hline $\begin{array}{l}\text { Reference } \\
\text { PEDro score }\end{array}$ & $\begin{array}{l}\text { Study } \\
\text { design } \\
\text { Allocated/ } \\
\text { Analysed }\end{array}$ & $\begin{array}{l}\text { Participants (n, } \\
\text { age mean (SD), \% } \\
\text { women, setting, } \\
\text { health status) }\end{array}$ & $\begin{array}{l}\text { Intervention } \\
\text { Primary exercise } \\
\text { type according to } \\
\text { ProFANE }^{\mathrm{a}}\end{array}$ & Control $^{\mathbf{b}}$ & Outcomes & $\begin{array}{l}\text { Follow } \\
\text { up } \\
\text { (mo) }\end{array}$ & Results \\
\hline & & $\begin{array}{l}\text { (HOME) } \\
\boldsymbol{n}=50 \\
\text { (randomised); } 47 \\
\text { (analysed) } \\
\text { Age: } 83 \text { (4) } \\
\text { Female: } 55 \%\end{array}$ & $\begin{array}{l}\text { Endurance training: } \\
\text { started from } 15 \text { mins } \\
\text { at } 65-75 \% \text { of peak } \\
\text { heart rate to } 30 \text { mins } \\
\text { at } 85-90 \% \text { of peak } \\
\text { heart rate } \\
\text { Session duration: NR } \\
\text { Delivered by: NR } \\
\text { Duration of the } \\
\text { intervention (wks): } \\
36 \\
\text { Primary exercise } \\
\text { type: Multiple } \\
\text { (resistance plus } \\
\text { balance/function plus } \\
\text { endurance) }\end{array}$ & & & & $\begin{array}{l}\text { A. Exercise: } 0.65 \pm \\
0.17 \\
\text { B. Control: } 0.58 \pm \\
0.12 \\
\text { 4. Lumbar spine (L2- } \\
\text { L4) BMD } \\
\text { A. Exercise: } 1.08 \pm \\
0.28 \\
\text { B. Control: } 0.97 \pm \\
0.23 \\
\text { 5. Whole Body BMD } \\
\text { A. Exercise: } 1.09 \pm \\
0.18 \\
\text { B. Control: } 1.03 \pm \\
0.17\end{array}$ \\
\hline $\begin{array}{l}\text { von Stengel } \\
2011 \\
7 / 10[78]\end{array}$ & $\begin{array}{l}\text { RCT } \\
151 / 141\end{array}$ & $\begin{array}{l}\text { Setting: } \\
\text { Community; } \\
\text { Germany } \\
\text { Health Status: } \\
\text { Healthy } \\
\text { A. Conventional } \\
\text { multicomponent } \\
\text { training } \\
\boldsymbol{n}=50 \\
\text { (randomised); } 47 \\
\text { (analysed) } \\
\text { Age: } 68.6 \text { (3) } \\
\text { B. Wellness } \\
\text { control } \\
\boldsymbol{n}=51 \\
\text { (randomised); } 48 \\
\text { (analysed) } \\
\text { Age: } 68.1 \text { (2.7) } \\
\text { Female: } 100 \%\end{array}$ & $\begin{array}{l}\text { A. Training sessions } \\
\text { consisting of aerobic } \\
\text { dancing; progressive } \\
\text { coordination and } \\
\text { balance training; } \\
\text { functional gymnastics } \\
\text { and isometric strength } \\
\text { training; and } \\
\text { progressive upper } \\
\text { body exercises. } \\
\text { Additionally, } \\
\text { participants were } \\
\text { requested to carry out } \\
\text { a home training } \\
\text { session. } \\
\text { Frequency: } 4 \text { times/ } \\
\text { week [i.e. } 2 \text { controlled } \\
\text { training session/week; } \\
2 \text { home exercise/ } \\
\text { week] } \\
\text { Intensity: } \\
\text { Dance aerobic: } 70- \\
80 \% \text { maximum heart } \\
\text { rate; } \\
\text { Functional gymnastics } \\
\text { and isometric } \\
\text { strength: 6-10s of max- } \\
\text { imum exertion and } \\
20-30 \text { s of active rest; } \\
\text { Upper body exercise: } 3 \\
\text { sets } 15 \text { reps } \\
\text { Session duration: } 60 \\
\text { min/training session; } \\
20 \text { min/home training } \\
\text { session } \\
\text { Delivered by: } \\
\text { Certified instructors } \\
\text { Duration of the } \\
\text { intervention (wks): } \\
72 \\
\text { Primary exercise } \\
\text { type: Multiple } \\
\text { (balance/function, plus } \\
\text { flexibility plus } \\
\text { resistance plus } \\
\text { endurance (dance) }\end{array}$ & $\begin{array}{l}\text { B: Low intensity } \\
\text { wellness } \\
\text { programme that } \\
\text { includes } \\
\text { light physical } \\
\text { exercises and a } \\
\text { relaxation } \\
\text { programme } \\
\text { Frequency: } 1 \text { time/ } \\
\text { week } \\
\text { Intensity: Light } \\
\text { Session duration: } \\
\text { NR } \\
\text { Delivered by: NR } \\
\text { Duration of the } \\
\text { intervention (wks): } \\
72 \\
\text { (10 weeks of } \\
\text { training were } \\
\text { intermitted by a } \\
\text { break of } 10 \text { weeks } \\
\text { and the training } \\
\text { cycle was repeated } \\
\text { throughout } 72 \\
\text { weeks) }\end{array}$ & $\begin{array}{l}\text { 1. Total hip BMD } \\
\text { 2. Lumbar spine } \\
\text { (L1-L4) BMD }\end{array}$ & 18 & $\begin{array}{l}\text { Mean difference } \\
(95 \% \mathrm{Cl}) \text { : } \\
\text { 1. Total hip BMD: } \\
0.002(-0.007 \text { to } \\
0.012) \\
\text { 2. Lumbar spine: } \\
0.015 \text { (0.001 to } \\
0.029)^{\mathrm{c}}\end{array}$ \\
\hline $\begin{array}{l}\text { dWinters- } \\
\text { Stone } 2014\end{array}$ & $\begin{array}{l}\text { RCT } \\
51 / 43\end{array}$ & $\begin{array}{l}\text { Setting: } \\
\text { Community; }\end{array}$ & $\begin{array}{l}\text { A. Progressive, } \\
\text { moderate-intensity }\end{array}$ & $\begin{array}{l}\text { B. FLEX Control: } \\
\text { Whole body }\end{array}$ & $\begin{array}{l}\text { 1. Lumbar spine } \\
\text { (L1-L4) BMD }\end{array}$ & 12 & $\begin{array}{l}\text { Final score (mean } \pm \\
\text { SD) }\end{array}$ \\
\hline
\end{tabular}


Table 1 Description of included studies comparing physical activity with a control intervention (Continued)

\begin{tabular}{|c|c|c|c|c|c|c|c|}
\hline $\begin{array}{l}\text { Reference } \\
\text { PEDro score }\end{array}$ & $\begin{array}{l}\text { Study } \\
\text { design } \\
\text { Allocated/ } \\
\text { Analysed }\end{array}$ & $\begin{array}{l}\text { Participants (n, } \\
\text { age mean (SD), \% } \\
\text { women, setting, } \\
\text { health status) }\end{array}$ & $\begin{array}{l}\text { Intervention } \\
\text { Primary exercise } \\
\text { type according to } \\
\text { ProFANE }^{\mathrm{a}}\end{array}$ & Control $^{\mathbf{b}}$ & Outcomes & $\begin{array}{l}\text { Follow } \\
\text { up } \\
(\mathrm{mo})\end{array}$ & Results \\
\hline $7 / 10$ [97] & & $\begin{array}{l}\text { United States } \\
\text { Health Status: } \\
\text { Prostate cancer } \\
\text { survivors receiving } \\
\text { androgen } \\
\text { deprivation } \\
\text { therapy without } \\
\text { osteoporosis } \\
\text { Age: } 70.2 \\
\text { A. Progressive, } \\
\text { moderate- } \\
\text { intensity resist- } \\
\text { ance + impact } \\
\text { training (POWIR) } \\
\text { n=29 } \\
\text { (randomised); } \\
24 \text { (analysed) } \\
\text { B. Control- } \\
\text { flexibility training } \\
\text { (FLEX) } \\
\text { n=22 } \\
\text { (randomised); } 12 \\
\text { (analysed) } \\
\text { Female: } 0 \%\end{array}$ & $\begin{array}{l}\text { resistance for upper } \\
\text { and lower body }+ \text { im- } \\
\text { pact training consisted } \\
\text { of two footed jumps } \\
\text { from the group to a } \\
\text { target height 1" from } \\
\text { the floor with a bent- } \\
\text { knee landing with } \\
\text { weighted vests } \\
\text { Frequency: } 3 \text { times/ } \\
\text { week (two supervised } \\
\text { classes + one home- } \\
\text { based session) } \\
\text { Intensity: Lower body } \\
\text { training } 1-2 \text { sets of } 8- \\
12 \text { reps from } 0 \% \text { to } \\
15 \% \text { of the body } \\
\text { weight. Upper body } \\
\text { training started from } \\
1-2 \text { sets of } 12-14 \text { reps } \\
\text { at } 13-15 \text { RM to 1-2 } \\
\text { sets of } 8 \text { to } 10 \text { reps at } \\
8-10 \text { RM. Jumping } \\
\text { started from 1-4 sets } \\
\text { of } 10 \text { reps at 0-1\% } \\
\text { body weight to } 9 \text { to } \\
10 \text { sets of } 10 \text { reps at } \\
10 \% \text { body weight } \\
\text { using weighted vest } \\
\text { Session duration: } 60 \\
\text { min } \\
\text { Delivered by: Trained } \\
\text { exercise instructors } \\
\text { Duration of the } \\
\text { intervention (wks): } \\
52 \\
\text { Primary exercise } \\
\text { type: Resistance with } \\
\text { bone loading }\end{array}$ & $\begin{array}{l}\text { stretching and } \\
\text { relaxation }\end{array}$ & $\begin{array}{l}\text { 2. Total hip BMD } \\
\text { 3. Greater } \\
\text { trochanter BMD } \\
\text { 4. Femoral neck } \\
\text { BMD }\end{array}$ & & $\begin{array}{l}\text { 1. Lumbar spine } \\
\text { BMD } \\
\text { A. POWIR: } 1.123 \pm \\
0.241 \\
\text { B. FLEX: } 1.094 \pm \\
0.156 \\
\text { 2. Total hip BMD } \\
\text { A. POWIR: } 0.956 \pm \\
0.135 \\
\text { B. FLEX: } 0.971 \pm \\
\text { 0.129 } \\
\text { 3. Greater trochanter } \\
\text { BMD } \\
\text { A. POWIR: } 0.776 \pm \\
\text { 0.131 } \\
\text { B. FLEX: } 0.783 \pm \\
\text { 0.112 } \\
\text { 4. Femoral neck } \\
\text { BMD } \\
\text { A. POWIR: } 0.752 \pm \\
\text { 0.131 } \\
\text { B. FLEX: } 0.791 \pm \\
\text { 0.098 }\end{array}$ \\
\hline $\begin{array}{l}\text { Woo } 2007 \\
6 / 10[75]\end{array}$ & $\begin{array}{l}\text { RCT } \\
180 / 176\end{array}$ & $\begin{array}{l}\text { Setting: } \\
\text { Community; Hong } \\
\text { Kong } \\
\text { Heath status: } \\
\text { Healthy } \\
\text { A. Tai Chi } \\
\mathbf{n}=60 \\
\text { (randomised); } 58 \\
\text { (randomised) } \\
\text { Age: } 68.2 \text { years } \\
\text { B. Resistance } \\
\text { training } \\
\boldsymbol{n}=60 \\
\text { (randomised); } 59 \\
\text { (randomised) } \\
\text { Age: } 68.7 \text { years } \\
\text { C. No Treatment } \\
\boldsymbol{n}=60 \\
\text { (randomised); } 59 \\
\text { (randomised) } \\
\text { Age: } 68.1 \text { years } \\
\text { Female: } 50 \%\end{array}$ & $\begin{array}{l}\text { A. Tai Chi: } 24-\text { forms } \\
\text { of Tai Chi using Yang } \\
\text { style } \\
\text { Frequency: } 3 \text { times/ } \\
\text { week } \\
\text { Intensity: NR } \\
\text { Session duration: NR } \\
\text { Delivered by: NR } \\
\text { Duration of the } \\
\text { intervention (wks): } \\
52 \text { weeks } \\
\text { Primary exercise } \\
\text { type: } 3 D \text { (Tai Chi) } \\
\text { B. Resistance } \\
\text { training: Resistance } \\
\text { training with the use } \\
\text { of medium strength } \\
\text { TheraBand } \\
\text { Frequency: } 3 \text { times/ } \\
\text { week } \\
\text { Intensity: } 30 \text { times } \\
\text { with medium strength } \\
\text { TheraBand } \\
\text { Session duration: NR } \\
\text { Delivered by: NR } \\
\text { Duration of the }\end{array}$ & C. No intervention & $\begin{array}{l}\text { 1. Total hip BMD } \\
\text { 2. Total spine BMD }\end{array}$ & 12 & $\begin{array}{l}\text { Change score (mean } \\
\% \text { change } \pm \text { SE) } \\
\text { Men } \\
\text { 1. Total hip BMD } \\
\text { A. Tai Chi: }-0.48 \pm \\
0.37 \\
\text { B. Resistance: }-1.20 \pm \\
0.38 \\
\text { C. Control: }-0.15 \pm \\
\text { 0.38 } \\
\text { 2. Total spine BMD } \\
\text { A. Tai Chi: } 1.35 \pm \\
\text { 0.40 } \\
\text { B. Resistance: } 1.27 \pm \\
\text { 0.42 } \\
\text { C. Control: } 0.54 \pm \\
\text { 0.42 } \\
\text { Women } \\
\text { 1. Total hip BMD } \\
\text { A. Tai Chi: } 0.07 \pm \\
\text { 0.64 } \\
\text { B. Resistance: } 0.09 \pm \\
\text { 0.62 } \\
\text { C. Control: }-2.25 \pm \\
\text { 0.60 } \\
\text { 2. Total spine BMD }\end{array}$ \\
\hline
\end{tabular}


Table 1 Description of included studies comparing physical activity with a control intervention (Continued)

\begin{tabular}{|c|c|c|c|c|c|c|c|}
\hline $\begin{array}{l}\text { Reference } \\
\text { PEDro score }\end{array}$ & $\begin{array}{l}\text { Study } \\
\text { design } \\
\text { Allocated/ } \\
\text { Analysed }\end{array}$ & $\begin{array}{l}\text { Participants ( } n \text {, } \\
\text { age mean (SD), \% } \\
\text { women, setting, } \\
\text { health status) }\end{array}$ & $\begin{array}{l}\text { Intervention } \\
\text { Primary exercise } \\
\text { type according to } \\
\text { ProFANE }^{\mathrm{a}}\end{array}$ & Control $^{\mathbf{b}}$ & Outcomes & $\begin{array}{l}\text { Follow } \\
\text { up } \\
\text { (mo) }\end{array}$ & Results \\
\hline & & & $\begin{array}{l}\text { intervention (wks): } \\
52 \text { weeks } \\
\text { Primary exercise } \\
\text { type: Resistance }\end{array}$ & & & & $\begin{array}{l}\text { A. Tai Chi: } 0.10 \pm \\
0.50 \\
\text { B. Resistance: } 1.98 \pm \\
0.48 \\
\text { C. Control: } 0.98 \pm \\
0.47\end{array}$ \\
\hline $\begin{array}{l}\text { Yoo } 2010 \\
4 / 10[76]\end{array}$ & $\begin{array}{l}\text { RCT } \\
28 / 21\end{array}$ & $\begin{array}{l}\text { Setting: } \\
\text { Community; Korea } \\
\text { Health status: } \\
\text { Healthy } \\
\text { A. Exercise } \\
\boldsymbol{n}=14 \\
\text { (randomised); } 11 \\
\text { (analysed) } \\
\text { Age: } 70.9 \text { (2.7) } \\
\text { B. Control } \\
\boldsymbol{n}=14 \\
\text { (randomised); } 10 \\
\text { (analysed) } \\
\text { Age: } 71.1(2.7) \\
\text { Female: } 100 \%\end{array}$ & $\begin{array}{l}\text { A. Supervised walking } \\
\text { exercise programme } \\
\text { involving walking } \\
\text { ankle weights. } \\
\text { Frequency: } 3 \text { times/ } \\
\text { week } \\
\text { Intensity: Maintained } \\
\text { at } 60 \% \text { of heart rate } \\
\text { reserve } \\
\text { Session duration: } 60 \\
\text { min } \\
\text { Delivered by: NR } \\
\text { Duration of the } \\
\text { intervention (wks): } \\
12 \\
\text { Primary exercise } \\
\text { type: Endurance } \\
\text { (walking with ankle } \\
\text { weights) }\end{array}$ & B. Control & $\begin{array}{l}\text { 1. Femoral neck } \\
\text { BMD } \\
\text { 2. Femoral Ward's } \\
\text { BMD } \\
\text { 3. Femoral } \\
\text { trochanter BMD } \\
\text { 4. Spine BMD } \\
\text { 5. Whole body } \\
\text { BMD }\end{array}$ & 3 & $\begin{array}{l}\text { Final score (mean } \pm \\
\text { SD) } \\
\text { 1. Femoral neck } \\
\text { BMD } \\
\text { A. Exercise: } 0.770 \pm \\
0.132 \\
\text { B. Control: } 0.729 \pm \\
0.124 \\
\text { 2. Femoral Ward's } \\
\text { BMD } \\
\text { A. Exercise: } 0.580 \pm \\
\text { 0.158 } \\
\text { B. Control: } 0.584 \pm \\
\text { 0.164 } \\
\text { 3. Femoral } \\
\text { trochanter BMD } \\
\text { A. Exercise: } 0.708 \pm \\
\text { 0.105 } \\
\text { B. Control: } 0.687 \pm \\
\text { 0.136 } \\
\text { 4. Spine BMD } \\
\text { A. Exercise: } 1.056 \pm \\
\text { 0.188 } \\
\text { B. Control: } 1.010 \pm \\
\text { 0.167 } \\
\text { 5. Whole body BMD } \\
\text { A. Exercise: } 1.057 \pm \\
\text { 0.077 } \\
\text { B. Control: } 1.028 \pm \\
\text { 0.109 }\end{array}$ \\
\hline
\end{tabular}

$B M C$ bone mineral content $(\mathrm{g}), B M D$ bone mineral density $\left(\mathrm{g} / \mathrm{cm}^{2}\right.$ unless specified), $B M I$ body mass index $\left(\mathrm{kg} / \mathrm{m}^{2}\right), B W$ bone width, $N R$ not reported, $R C T$ randomised controlled trial. In studies where other groups or other outcomes not of interest to this study were included (example supplement, or whole-body vibration) we only included and extracted information for the groups and for the comparisons that were relevant to this study (i.e., those where the effect of physical activity could be evaluated). When data was available for more than one time-point, we extracted the post-intervention data and any additional followup. Mean estimates were extracted in the following hierarchical order: mean difference, change score and final score

${ }^{a}$ Exercise is a physical activity that is planned, structured and repetitive and aims to improve or maintain physical fitness. There is a wide range of possible types of exercise, and exercise programmes often include one or more types of exercise. We categorised exercise based on a modification of the Prevention of Falls Network Europe (ProFaNE) taxonomy that classifies exercise type as: i) gait, balance, and functional training; ii) strength/ resistance (including power); iii) flexibility; iv) threedimensional (3D) exercise (e.g., Tai Chi, Qigong, dance); v) general physical activity; vi) endurance; and vii) other kind of exercises. The taxonomy allows for more than one type of exercise to be delivered within a programme. We also considered whether the exercise explicitly included bone loading eg hopping or heel drops ${ }^{\mathrm{b}} \mathrm{A}$ control intervention is one that is not thought to improve bone health, such as general health education, social visits, very gentle exercise, or 'sham' exercise not expected to impact on bone health.

cindicates statistically significant between-group differences at $p<0.05$

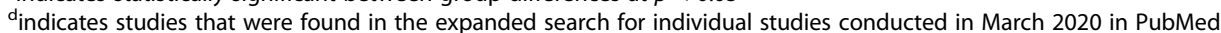

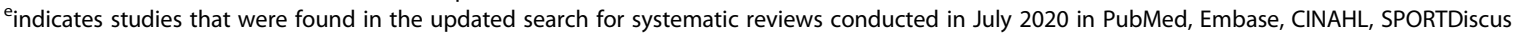

$\S$ and $\neq$ and $\uparrow$ indicate articles reporting results from the same study

and targeted the lower limb, hip measures were preferred. For studies that reported multiple hip measures, preference was given to total hip measures, if available. Preference was given to BMD when compared to other measures, such as BMC. We undertook two additional assessments according to the two most commonly reported outcomes across the included studies, which were measures of femoral neck BMD and lumbar spine BMD.

\section{Methodological quality of studies}

The overall quality of included trials was moderate (median 5 , range 1 to 7 ). The PEDro total scores are reported for all relevant studies in Tables 1, 2, and 3 and the scores for each item are reported in Additional file 4, Table 1. The overall risk of bias of longitudinal studies using the modified QUIPS tool is reported in Table 4. Six longitudinal studies had low risk of bias (Additional file 4, Table 2). The most common sources of bias were 
Table 2 Description of included studies comparing two or more forms of physical activity

\begin{tabular}{|c|c|c|c|c|c|c|c|}
\hline $\begin{array}{l}\text { Reference } \\
\text { PEDro score }\end{array}$ & $\begin{array}{l}\text { Study } \\
\text { design } \\
\text { Allocated/ } \\
\text { Analysed }\end{array}$ & $\begin{array}{l}\text { Participants } \\
\text { (n, age mean } \\
\text { (SD), \% } \\
\text { women, } \\
\text { setting, } \\
\text { health status) }\end{array}$ & $\begin{array}{l}\text { Intervention } \\
\text { Primary exercise type } \\
\text { according to ProFANE }^{\mathrm{a}}\end{array}$ & $\begin{array}{l}\text { Relevant } \\
\text { comparison }\end{array}$ & Outcomes & $\begin{array}{l}\text { Follow } \\
\text { up } \\
(\mathrm{mo})\end{array}$ & Results \\
\hline $\begin{array}{l}{ }^{\mathrm{d} A r m a m e n t o-} \\
\text { Villareal } 2020 \\
7 / 10[87]\end{array}$ & $\begin{array}{l}\text { RCT } \\
160 / 141\end{array}$ & $\begin{array}{l}\text { Setting: } \\
\text { Community; } \\
\text { United States } \\
\text { Health status: } \\
\text { Obese older } \\
\text { adults } \\
\text { A. Aerobic } \\
\text { exercise } \\
\boldsymbol{n}=40 \\
\text { (randomised); } \\
35 \text { (analysed) } \\
\text { Age: } 70 \text { (4) } \\
\text { Female: 65\% } \\
\text { B. Resistance } \\
\text { exercise } \\
\boldsymbol{n}=40 \\
\text { (randomised); } \\
35 \text { (analysed) } \\
\text { Age: } 70 \text { (5) } \\
\text { Female: 63\% } \\
\text { C. } \\
\text { Combination } \\
\boldsymbol{n}=40 \\
\text { (randomised); } \\
35 \text { (analysed) } \\
\text { 40 } \\
\text { Age: } 70 \text { (5) } \\
\text { Female: 60\% } \\
\text { D. Control } \\
\text { [control group } \\
\text { not relevant } \\
\text { for this review } \\
\text { question] }\end{array}$ & $\begin{array}{l}\text { A. Aerobic exercise } \\
\text { Frequency: } 3 \text { times/week } \\
\text { Intensity: } 65 \% \text { of peak heart } \\
\text { rate, gradually increased to } 70 \% \\
\text { to } 85 \% \text {. } \\
\text { Session duration: } \sim 60 \text { min } \\
\text { Delivered by: Exercise } \\
\text { physiologists } \\
\text { Duration of the intervention } \\
\text { (wks): } 26 \\
\text { Primary exercise type: } \\
\text { Endurance } \\
\text { B. Resistance exercise: nine } \\
\text { upper-body and lower-body ex- } \\
\text { ercises using weightlifting } \\
\text { machines } \\
\text { Frequency: } 3 \text { times/week } \\
\text { Intensity: } 1 \text { to } 2 \text { sets of } 8 \text { to } 12 \\
\text { reps at } 65 \% \text { of the } 1 \text { RM and } \\
\text { increased progressively to } 2 \text { to } \\
3 \text { sets at } 85 \% \text { of the } 1 \text {-RM. } \\
\text { Session duration: } 60 \text { min } \\
\text { Delivered by: Exercise } \\
\text { physiologists } \\
\text { Duration of the intervention } \\
\text { (wks): } 26 \\
\text { Primary exercise type: } \\
\text { Resistance } \\
\text { C. Combination: Aerobic and } \\
\text { resistance exercise training } \\
\text { sessions } \\
\text { Frequency: } 3 \text { times/week } \\
\text { Intensity: } \\
\text { Aerobic exercise: } ~ 65 \% \text { of their } \\
\text { peak heart rate, gradually } \\
\text { increased to } 70 \% \text { to } 85 \% \text {. } \\
\text { Resistance exercise: } 1 \text { to } 2 \text { sets } \\
\text { of } 8 \text { to } 12 \text { reps at } 65 \% \text { of the } 1 \\
\text { RM and increased progressively } \\
\text { to } 2 \text { to } 3 \text { sets at } ~ 85 \% \text { of the } 1- \\
\text { RM } \\
\text { Session duration: } 75 \text { to } 90 \text { min } \\
\text { Delivered by: Exercise } \\
\text { physiologists } \\
\text { Duration of the intervention } \\
\text { (wks): } 26 \\
\text { Primary exercise type: } \\
\text { Multiple (endurance plus } \\
\text { resistance) }\end{array}$ & $\begin{array}{l}A \text { vs } B \\
A \text { vs C } \\
B \text { vs C }\end{array}$ & $\begin{array}{l}\text { 1. Total hip } \\
\text { BMD } \\
\text { 2. Femoral } \\
\text { neck BMD } \\
\text { 3. Trochanter } \\
\text { BMD } \\
\text { 4. } \\
\text { Intertrochanter } \\
\text { BMD } \\
\text { 5. Lumbar } \\
\text { spine BMD } \\
\text { 6. Whole body } \\
\text { BMD } \\
\text { 7. One-third ra- } \\
\text { dius BMD }\end{array}$ & 6 & 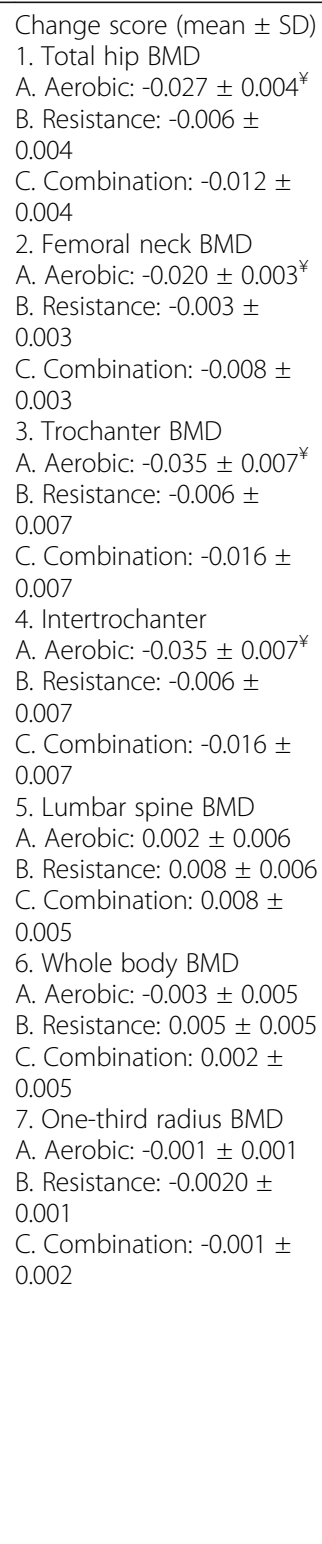 \\
\hline $\begin{array}{l}\text { Ashe } 2013 \\
\text { [77] }\end{array}$ & $\begin{array}{l}\text { RCT } \\
155 / 135\end{array}$ & $\begin{array}{l}\text { Setting: } \\
\text { Community; } \\
\text { Canada } \\
\text { Health status: } \\
\text { Healthy } \\
\text { A. Balance } \\
\text { and tone (BT) } \\
\boldsymbol{n}=49 \\
\text { (randomised); } \\
42 \text { (analysed) }\end{array}$ & $\begin{array}{l}\text { A. BT: Group-based supervised } \\
\text { intervention consisting of bal- } \\
\text { ance and tone training with the } \\
\text { use of body weight. } \\
\text { Frequency: } 2 \text { times/week } \\
\text { Intensity: NR } \\
\text { Session duration: NR } \\
\text { Primary exercise type: Balance } \\
\text { and functional } \\
\text { B. RT1: Low-frequency, group- }\end{array}$ & $\begin{array}{l}A \text { vs B } \\
A \text { vs C }\end{array}$ & $\begin{array}{l}\text { 1. Tibial } \\
\text { volumetric } \\
\text { cortical density } \\
\text { (CovBMD) } \\
\text { 2. Total area } \\
\text { (ToA) midtibia } \\
\text { 3. Tibial bone } \\
\text { strength }\end{array}$ & 12 & $\begin{array}{l}\text { Adjusted mean difference } \\
(95 \% \text { Cl) } \\
\text { 1. Tibial CovBMD } \\
\text { B - A } \\
0.76(-5.32 \text { to } 6.85) \\
\text { C - A } \\
-2.09 \text { (-8.22 to } 4.05) \\
\text { 2. Total area (ToA) midtibia } \\
\text { B - A } \\
0.10 \text { (-2.72 to } 2.92)\end{array}$ \\
\hline
\end{tabular}


Table 2 Description of included studies comparing two or more forms of physical activity (Continued)

\begin{tabular}{|c|c|c|c|c|c|c|c|}
\hline $\begin{array}{l}\text { Reference } \\
\text { PEDro score }\end{array}$ & $\begin{array}{l}\text { Study } \\
\text { design } \\
\text { Allocated/ } \\
\text { Analysed }\end{array}$ & $\begin{array}{l}\text { Participants } \\
\text { (n, age mean } \\
\text { (SD), \% } \\
\text { women, } \\
\text { setting, } \\
\text { health status) }\end{array}$ & $\begin{array}{l}\text { Intervention } \\
\text { Primary exercise type } \\
\text { according to ProFANE }^{\mathrm{a}}\end{array}$ & $\begin{array}{l}\text { Relevant } \\
\text { comparison }\end{array}$ & Outcomes & $\begin{array}{l}\text { Follow } \\
\text { up } \\
(\mathrm{mo})\end{array}$ & Results \\
\hline & & $\begin{array}{l}\text { Age: } 69.9(3.1) \\
\text { B. Once a } \\
\text { week } \\
\text { resistance } \\
\text { training (RT1) } \\
\boldsymbol{n}=54 \\
\text { (randomised); } \\
47 \text { (analysed) } \\
\text { Age: } 69.4 \text { (3.0) } \\
\text { C. Twice a } \\
\text { week } \\
\text { resistance } \\
\text { training (RT2) } \\
\boldsymbol{n}=52 \\
\text { (randomised); } \\
46 \text { (analysed) } \\
\text { Age: } 69.2 \text { (3.0) } \\
\text { Female: 100\% }\end{array}$ & $\begin{array}{l}\text { based supervised resistance } \\
\text { training for upper and lower } \\
\text { body with the use of resistance } \\
\text { equipment. } \\
\text { Frequency: } 1 \text { time/week } \\
\text { Intensity: } 2 \text { sets of } 8 \text { RM } \\
\text { Session duration: NR } \\
\text { Primary exercise type: } \\
\text { Resistance } \\
\text { C. RT2: High-frequency, group- } \\
\text { based supervised resistance } \\
\text { training for upper and lower } \\
\text { body with the use of resistance } \\
\text { equipment. } \\
\text { Frequency: } 2 \text { times/week } \\
\text { Intensity: } 2 \text { sets of } 8 \text { RM } \\
\text { Session duration: NR } \\
\text { Primary exercise type: } \\
\text { Resistance } \\
\text { Duration of the interventions } \\
\text { (wks): } 52 \\
\text { Delivered by: Certified fitness } \\
\text { instructors }\end{array}$ & & & & $\begin{array}{l}\text { C - A } \\
-0.49(-3.34 \text { to } 2.35) \\
\text { 3. Tibial bone strength } \\
\text { B - A } \\
23.32(-248.86 \text { to } 295.5) \\
\text { C - A } \\
-91.56(-366.5 \text { to } 183.28)\end{array}$ \\
\hline $\begin{array}{l}{ }^{\text {b} B l u m e n t h a l ~} \\
1991 \\
6 / 10[44]\end{array}$ & $\begin{array}{l}\text { RCT } \\
101 / 85\end{array}$ & $\begin{array}{l}\text { Setting: NR } \\
\text { Health status: } \\
\text { Healthy } \\
\text { A. Aerobic } \\
\text { Training } \\
\boldsymbol{n}=33 \\
\text { (randomised) } \\
\text { B. Yoga and } \\
\text { flexibility } \\
\boldsymbol{n}=34 \\
\text { (randomised) } \\
\text { C. Control: } \\
\text { Not relevant } \\
\text { for this } \\
\text { comparison. } \\
\text { Age: (whole } \\
\text { sample) } 67 \\
\text { (min-max: } 60- \\
\text { 83) } \\
\text { Female: NR }\end{array}$ & $\begin{array}{l}\text { A. Aerobic training: } \\
\text { Endurance training involving } \\
\text { bicycle ergometry, brisk } \\
\text { walking/jogging, and arm } \\
\text { ergometry. } \\
\text { Frequency: } 3 \text { times/week } \\
\text { Intensity: } 70 \% \text { heart rate } \\
\text { reserve } \\
\text { Session duration: } 60 \text { min } \\
\text { Delivered by: NR } \\
\text { Duration of intervention } \\
\text { (wks): } 16 \\
\text { Primary exercise type: } \\
\text { Endurance training } \\
\text { B. Yoga: Supervised non- } \\
\text { aerobic yoga programme. } \\
\text { Frequency: at least } 2 \text { times/ } \\
\text { week } \\
\text { Intensity: NR } \\
\text { Session duration: } 60 \text { min } \\
\text { Delivered by: NR } \\
\text { Duration of intervention } \\
\text { (wks): } 16 \\
\text { Primary exercise type: Balance } \\
\text { and function }\end{array}$ & $A$ vs $B$ & $\begin{array}{l}\text { 1. Distal radius } \\
\text { BMD }\end{array}$ & 14 & $\begin{array}{l}\text { 1. Distal radius BMD: no } \\
\text { between-group differences. } \\
\text { Quantitative estimates not } \\
\text { reported for between- } \\
\text { group comparisons. }\end{array}$ \\
\hline $\begin{array}{l}{ }^{d} \text { Chan } 2018 \\
7 / 10[92]\end{array}$ & $\begin{array}{l}\text { RCT } \\
110 / 54\end{array}$ & $\begin{array}{l}\text { Setting: } \\
\text { Community; } \\
\text { Taiwan } \\
\text { Health Status: } \\
\text { Increased risk } \\
\text { for falls and } \\
\text { fracture } \\
\text { A. Integrated } \\
\text { care (IC) } \\
\boldsymbol{n}=55\end{array}$ & $\begin{array}{l}\text { Both groups: 1-hour educa- } \\
\text { tional course related to osteo- } \\
\text { porosis, sarcopenia and 1-hour } \\
\text { exercise intervention including } \\
\text { warm up, brisk walking and } \\
\text { gentle stretching. Subjects en- } \\
\text { couraged to conduct exercise } \\
\text { at least } 3 \text { times per week. } \\
\text { A. Integrated care (IC): Basic } \\
\text { intervention, 15-minute warm- }\end{array}$ & $A$ vs $B$ & $\begin{array}{l}\text { 1. Lumbar } \\
\text { spine BMD } \\
\text { 2. Hip BMD }\end{array}$ & 3 & $\begin{array}{l}\text { Change score (\% change) } \\
\text { 1. Lumbar spine BMD } \\
\text { A. IC: } 1.26 \% \\
\text { B. LEE: } 2.08 \% \\
\text { 2. Hip BMD } \\
\text { A. IC: }-1.73 \% \\
\text { B. LEE: }-0.88 \%\end{array}$ \\
\hline
\end{tabular}


Table 2 Description of included studies comparing two or more forms of physical activity (Continued)

\begin{tabular}{|c|c|c|c|c|c|c|c|}
\hline $\begin{array}{l}\text { Reference } \\
\text { PEDro score }\end{array}$ & $\begin{array}{l}\text { Study } \\
\text { design } \\
\text { Allocated/ } \\
\text { Analysed }\end{array}$ & $\begin{array}{l}\text { Participants } \\
\text { (n, age mean } \\
\text { (SD), \% } \\
\text { women, } \\
\text { setting, } \\
\text { health status) }\end{array}$ & $\begin{array}{l}\text { Intervention } \\
\text { Primary exercise type } \\
\text { according to ProFANE }^{\mathrm{a}}\end{array}$ & $\begin{array}{l}\text { Relevant } \\
\text { comparison }\end{array}$ & Outcomes & $\begin{array}{l}\text { Follow } \\
\text { up } \\
(\mathrm{mo})\end{array}$ & Results \\
\hline & & $\begin{array}{l}\text { (randomised); } \\
31 \text { (analysed) } \\
\text { Age: } 74.6(7.4) \\
\text { Female: 69\% } \\
\text { B. Lower } \\
\text { extremity } \\
\text { exercise (LEE) } \\
\boldsymbol{n}=55 \\
\text { (randomised); } \\
23 \text { (analysed) } \\
\text { Age: } 73.08 \\
\text { (6.57) } \\
\text { Female: } 69 \%\end{array}$ & $\begin{array}{l}\text { up exercise, } 30 \text {-minute resist- } \\
\text { ance exercise and } 10 \text {-minute } \\
\text { balance exercise } \\
\text { Frequency: } 1 \text { time/week } \\
\text { Intensity: Resistance training } \\
\text { using rubber band and bottled } \\
\text { water }(0.6-1 \mathrm{~L} \text { ) as weight for } \\
\text { upper and lower limbs. } \\
\text { Delivered by: NR } \\
\text { Session duration: } 55 \text { min } \\
\text { Duration of the intervention } \\
\text { (wks): } 12 \\
\text { Primary exercise type: } \\
\text { Multiple (resistance plus } \\
\text { balance and functional) } \\
\text { B. Lower extremity exercise } \\
\text { (LEE): Basic intervention and } \\
\text { machine based lower extremity } \\
\text { resistance exercise } \\
\text { Frequency: } 2 \text { times/week } \\
\text { Intensity: } 60-80 \% \text { of } 1 \text { RM } \\
\text { Delivered by: NR } \\
\text { Session duration: } 30 \text { min } \\
\text { Duration of the intervention } \\
\text { (wks): } 12 \\
\text { Primary exercise type: } \\
\text { Resistance }\end{array}$ & & & & \\
\hline $\begin{array}{l}{ }^{b} \text { Helge } 2014 \\
5 / 10[50]\end{array}$ & $\begin{array}{l}\text { RCT } \\
27 / 23\end{array}$ & $\begin{array}{l}\text { Setting: } \\
\text { Community; } \\
\text { Denmark } \\
\text { Health status: } \\
\text { Healthy } \\
\text { A. Football } \\
\text { group } \\
\boldsymbol{n}=9 \\
\text { (randomised); } \\
9 \text { (analysed) } \\
\text { Age: } 68.0 \text { (4.0) } \\
\text { B. Resistance } \\
\text { training } \\
\boldsymbol{n}=9 \\
\text { (randomised); } \\
8 \text { (analysed) } \\
\text { Age: } 69.1 \text { (3.1) } \\
\text { C. Control: } \\
\text { Not relevant } \\
\text { for this } \\
\text { comparison. } \\
\text { Female: } 0 \%\end{array}$ & $\begin{array}{l}\text { A. Football group: Supervised } \\
\text { progressive football training } \\
\text { Frequency: } 1.7 \text { (0.3) times/week } \\
\text { (range: } 1.2-2.2 \text { ) } \\
\text { Intensity: } 82 \% \text { of maximum } \\
\text { heart rate (range } 64 \text { to } 90 \% \text { ) } \\
\text { Session duration: } 45 \text { to } 60 \text { min } \\
\text { Delivered by: NR } \\
\text { Duration of the intervention } \\
\text { (wks): } 52 \\
\text { Primary exercise type: Balance } \\
\text { and function (football) } \\
\text { B. Resistance training: } \\
\text { Progressive resistance training } \\
\text { for core and upper and lower } \\
\text { body } \\
\text { Frequency: } 1.9 \text { (0.2) times/week } \\
\text { (range: } 1.4-2.2 \text { ) } \\
\text { Intensity: Started from } 3 \text { sets of } \\
16-20 \text { RM to } 4 \text { sets of } 8 \text { RM } \\
\text { Session duration: } 45 \text { to } 60 \text { min } \\
\text { Delivered by: NR } \\
\text { Duration of the intervention } \\
\text { (wks): } 52 \\
\text { Primary exercise type: } \\
\text { Resistance (seated) }\end{array}$ & $A$ vs $B$ & $\begin{array}{l}\text { 1. Whole body } \\
\text { BMD } \\
\text { 2. Right } \\
\text { femoral neck } \\
\text { BMD } \\
\text { 3. Left femoral } \\
\text { neck BMD } \\
\text { 4. Right } \\
\text { femoral shaft } \\
\text { BMD } \\
\text { 5. Left femoral } \\
\text { shaft BMD } \\
\text { 6. Total right } \\
\text { proximal femur } \\
\text { 7. Total left } \\
\text { proximal femur }\end{array}$ & 12 & $\begin{array}{l}\text { Final score (mean } \pm \text { SD) } \\
\text { 1. Whole body BMD } \\
\text { A. Football: } 1.211 \pm 0.036 \\
\text { B. Resistance: } 1.225 \pm 0.024 \\
\text { 2. Right femoral neck BMD } \\
\text { A. Football: } 0.921 \pm 0.034 \\
\text { B. Resistance: } 1.000 \pm 0.042 \\
\text { 3. Left femoral neck BMD } \\
\text { A. Football: } 0.939 \pm 0.034 \\
\text { B. Resistance: } 1.006 \pm 0.036 \\
\text { 4. Right femoral shaft BMD } \\
\text { A. Football: } 1.156 \pm 0.042 \\
\text { B. Resistance: } 1.229 \pm 0.056 \\
\text { 5. Left femoral shaft BMD } \\
\text { A. Football: } 1.143 \pm 0.043 \\
\text { B. Resistance: } 1.229 \pm 0.057 \\
\text { 6. Total right proximal } \\
\text { femur } \\
\text { A. Football: } 0.982 \pm 0.031 \\
\text { B. Resistance: } 1.066 \pm 0.048 \\
\text { 7. Total left proximal femur } \\
\text { A. Football: } 0.989 \pm 0.031 \\
\text { B. Resistance: } 1.069 \pm 0.048\end{array}$ \\
\hline $\begin{array}{l}{ }^{\text {bKarinkanta }} \\
2007^{c} \\
7 / 10[53]\end{array}$ & $\begin{array}{l}\text { RCT } \\
149 / 144\end{array}$ & $\begin{array}{l}\text { Setting: } \\
\text { Community; } \\
\text { Finland } \\
\text { Health Status: } \\
\text { Healthy and }\end{array}$ & $\begin{array}{l}\text { A. Balance-jumping training: } \\
\text { Balance training including static } \\
\text { and dynamic balance exercise, } \\
\text { agility training, impact exercises } \\
\text { and changes of direction }\end{array}$ & $\begin{array}{l}A \text { vs } B \\
A \text { vs } C \\
B \text { vs } C\end{array}$ & $\begin{array}{l}\text { 1. Femoral } \\
\text { neck BMC } \\
\text { 2. Distal tibia } \\
\text { trabecular } \\
\text { density (mg/ }\end{array}$ & 12 & $\begin{array}{l}\text { Final score (mean } \pm \text { SD) } \\
\text { 1. Femoral neck BMC } \\
\text { A. Balance: } 2.73 \pm 0.40 \\
\text { B. Resistance: } 2.71 \pm 0.33 \\
\text { C. Combined: } 2.65 \pm 0.29\end{array}$ \\
\hline
\end{tabular}


Table 2 Description of included studies comparing two or more forms of physical activity (Continued)

\begin{tabular}{|c|c|c|c|c|c|c|c|}
\hline $\begin{array}{l}\text { Reference } \\
\text { PEDro score }\end{array}$ & $\begin{array}{l}\text { Study } \\
\text { design } \\
\text { Allocated/ } \\
\text { Analysed }\end{array}$ & $\begin{array}{l}\text { Participants } \\
\text { (n, age mean } \\
\text { (SD), \% } \\
\text { women, } \\
\text { setting, } \\
\text { health status) }\end{array}$ & $\begin{array}{l}\text { Intervention } \\
\text { Primary exercise type } \\
\text { according to ProFANE }^{\mathrm{a}}\end{array}$ & $\begin{array}{l}\text { Relevant } \\
\text { comparison }\end{array}$ & Outcomes & $\begin{array}{l}\text { Follow } \\
\text { up } \\
(\mathrm{mo})\end{array}$ & Results \\
\hline & & $\begin{array}{l}\text { excluded } \\
\text { participants } \\
\text { with } \\
\text { osteoporosis } \\
\text { A. Balance- } \\
\text { jumping } \\
\text { training } \\
\boldsymbol{n}=37 \\
\text { (randomised); } \\
35 \text { (analysed) } \\
\text { Age: } 72.9 \text { (2.3) } \\
\text { B. Resistance } \\
\text { training } \\
\boldsymbol{n}=37 \\
\text { (randomised); } \\
37 \text { (analysed) } \\
\text { Age: } 72.7 \text { (2.5) } \\
\text { C. Combined } \\
\text { Balance- } \\
\text { jumping and } \\
\text { resistance } \\
\text { training } \\
\boldsymbol{n}=38 \\
\text { (randomised); } \\
36 \text { (analysed) } \\
\text { Age: } 72.9 \text { (2.2) } \\
\text { D. Control: } \\
\text { Not relevant } \\
\text { for this } \\
\text { comparison } \\
\text { Female: } 100 \%\end{array}$ & $\begin{array}{l}\text { exercise. } \\
\text { Intensity: NR } \\
\text { Primary exercise type: Balance } \\
\text { and function including bone } \\
\text { loading (jumps) } \\
\text { B. Resistance training: } \\
\text { Tailored progressive resistance } \\
\text { training programme for large } \\
\text { muscle groups. } \\
\text { Intensity: Initially } 2 \text { sets of 10- } \\
15 \text { reps at intensity } 50-60 \% \text { of } \\
1 \text { RM, progressed to } 3 \text { sets of } 8 \text { - } \\
10 \text { reps at } 75-80 \% \text { of } 1 \text { RM. Rate } \\
\text { of perceived exertion: above } 18 \\
\text { out of } 20 \\
\text { Primary exercise type: } \\
\text { Resistance } \\
\text { C. Combined Balance- } \\
\text { jumping and resistance train- } \\
\text { ing: A combination of A \& B on } \\
\text { alternate weeks. } \\
\text { Primary exercise type: } \\
\text { Multiple (balance and function } \\
\text { plus resistance) } \\
\text { For all exercise groups: } \\
\text { Frequency: } 3 \text { times/week } \\
\text { Session duration: } 50 \text { min } \\
\text { Delivered by: Exercise leaders } \\
\text { Duration of the intervention } \\
\text { (wks): } 52\end{array}$ & & $\left.\mathrm{~cm}^{3}\right)$ & & $\begin{array}{l}\text { 2. Distal tibia trabecular } \\
\left.\text { density (mg/ } \mathrm{cm}^{3}\right) \\
\text { A. Balance: } 224 \pm 34 \\
\text { B. Resistance: } 219 \pm 26 \\
\text { C. Combined: } 215 \pm 39\end{array}$ \\
\hline $\begin{array}{l}{ }^{e b} \text { Karinkanta } \\
2009^{c} \\
5 / 10[98]\end{array}$ & $\begin{array}{l}\text { RCT } \\
149 / 126\end{array}$ & $\begin{array}{l}\text { Setting: } \\
\text { Community; } \\
\text { Finland } \\
\text { Health Status: } \\
\text { healthy and } \\
\text { excluded } \\
\text { participants } \\
\text { with } \\
\text { osteoporosis } \\
\text { A. Balance } \\
\text { jumping } \\
\text { training } \\
\text { group } \\
\boldsymbol{n}=37 \\
\text { (randomised); } \\
33 \text { (analysed) } \\
\text { Age: } 72.9 \text { (2.3) } \\
\text { B. Resistance } \\
\text { training } \\
\text { group } \\
\boldsymbol{n}=37 \\
\text { (randomised); } \\
34 \text { (analysed) } \\
\text { Age: } 72.7 \text { (2.5) } \\
\text { C. Combined } \\
\text { resistance } \\
\text { and balance } \\
\text { jumping }\end{array}$ & $\begin{array}{l}\text { A. Balance-jumping training: } \\
\text { Balance training including static } \\
\text { and dynamic balance exercise, } \\
\text { agility training, impact exercises } \\
\text { and changes of direction } \\
\text { exercise. } \\
\text { Intensity: NR } \\
\text { Primary exercise type: Balance } \\
\text { and function including bone } \\
\text { loading (jumps) } \\
\text { B. Resistance training: } \\
\text { Tailored progressive resistance } \\
\text { training programme for large } \\
\text { muscle groups. } \\
\text { Intensity: Initially } 2 \text { sets of } 10- \\
15 \text { reps at intensity } 50-60 \% \text { of } \\
1 \text { RM, progressed to } 3 \text { sets of } 8- \\
10 \text { reps at } 75-80 \% \text { of } 1 \text { RM. Rate } \\
\text { of perceived exertion: above } 18 \\
\text { out of } 20 \\
\text { Primary exercise type: } \\
\text { Resistance } \\
\text { C. Combined Balance- } \\
\text { jumping and resistance train- } \\
\text { ing: A combination of A \& B on } \\
\text { alternate weeks. } \\
\text { Primary exercise type: } \\
\text { Multiple (balance and function }\end{array}$ & $\begin{array}{l}\text { A vs D } \\
B \text { vs D } \\
\text { C vs D }\end{array}$ & $\begin{array}{l}\text { 1. Femoral } \\
\text { neck section } \\
\text { moduls }(\mathrm{Z}) \\
\left(\mathrm{mm}^{3}\right) \\
\text { 2. Tibia } \\
\text { midshaft } \\
\text { desnity- } \\
\text { weighted polar } \\
\text { section modu- } \\
\text { lus (BSI) }\left(\mathrm{mm}^{3}\right)\end{array}$ & 12 & $\begin{array}{l}\text { Mean change score }(95 \% \\
\text { Cl) reported on a graph } \\
\text { Quantitative data was only } \\
\text { reported between-group } \\
\text { differences with control } \\
\text { group as a reference. Add- } \\
\text { itional results were re- } \\
\text { ported in a graph. }\end{array}$ \\
\hline
\end{tabular}


Table 2 Description of included studies comparing two or more forms of physical activity (Continued)

\begin{tabular}{|c|c|c|c|c|c|c|c|}
\hline $\begin{array}{l}\text { Reference } \\
\text { PEDro score }\end{array}$ & $\begin{array}{l}\text { Study } \\
\text { design } \\
\text { Allocated/ } \\
\text { Analysed }\end{array}$ & $\begin{array}{l}\text { Participants } \\
\text { (n, age mean } \\
\text { (SD), \% } \\
\text { women, } \\
\text { setting, } \\
\text { health status) }\end{array}$ & $\begin{array}{l}\text { Intervention } \\
\text { Primary exercise type } \\
\text { according to ProFANE }^{\mathrm{a}}\end{array}$ & $\begin{array}{l}\text { Relevant } \\
\text { comparison }\end{array}$ & Outcomes & $\begin{array}{l}\text { Follow } \\
\text { up } \\
(\mathrm{mo})\end{array}$ & Results \\
\hline & & $\begin{array}{l}\text { training } \\
\text { group } \\
\boldsymbol{n}=38 \\
\text { (randomised); } \\
32 \text { (analysed) } \\
\text { Age: } 72.9(2.2) \\
\text { D. Non- } \\
\text { training con- } \\
\text { trol group } \\
\boldsymbol{n}=27 \\
\text { (randomised); } \\
27 \text { (analysed) } \\
\text { Age: } 72.0(2.1) \\
\text { Female: } 100 \%\end{array}$ & $\begin{array}{l}\text { plus resistance) } \\
\text { For all exercise groups: } \\
\text { Frequency: } 3 \text { times/week } \\
\text { Session duration: } 50 \text { min } \\
\text { Delivered by: Exercise leaders } \\
\text { Duration of the intervention } \\
\text { (wks): } 52\end{array}$ & & & & \\
\hline $\begin{array}{l}\text { bKohrt } 1997 \\
3 / 10[55]\end{array}$ & $\begin{array}{l}\text { Quasi- } \\
\text { randomised } \\
\text { trial } \\
39 / 30\end{array}$ & $\begin{array}{l}\text { Setting: NR; } \\
\text { United States } \\
\text { Health Status: } \\
\text { Healthy } \\
\text { A. Ground } \\
\text { reaction } \\
\text { forces } \\
\text { training } \\
\boldsymbol{n}=14 \\
\text { (randomised); } \\
12 \text { (analysed) } \\
\text { Age: } 66.0 \text { (1.0) } \\
\text { B. Joint } \\
\text { reaction } \\
\text { forces } \\
\text { training } \\
\boldsymbol{n}=13 \\
\text { (randomised); } \\
9 \text { (analysed) } \\
\text { Age: } 65.0 \text { (1.0) } \\
\text { C. Control: } \\
\text { Not relevant } \\
\text { for this } \\
\text { comparison } \\
\text { Female: 100\% }\end{array}$ & $\begin{array}{l}\text { A. Ground reaction forces } \\
\text { training: Individualised exercise } \\
\text { training focusing on activities } \\
\text { that involved ground-reaction } \\
\text { forces, such as walking, jogging } \\
\text { and/or stair climbing. } \\
\text { Frequency: } 3 \text { to } 5 \text { times/week } \\
\text { Intensity: } 60-70 \% \text { to } 80-85 \% \\
\text { maximum heart rate } \\
\text { Session duration: } 30 \text { to } 45 \\
\text { min } \\
\text { Delivered by: NR } \\
\text { Duration of the intervention } \\
\text { (wks): } 36 \\
\text { Primary exercise type: } \\
\text { Multiple (balance and function } \\
\text { plus endurance plus flexibility) } \\
\text { B. Joint reaction forces } \\
\text { training: Individualised exercise } \\
\text { training including activities that } \\
\text { involved joint-reaction forces, } \\
\text { such as weightlifting and } \\
\text { rowing. } \\
\text { Frequency: } 3 \text { to } 5 \text { sessions/ } \\
\text { week } \\
\text { Intensity: Weightlifting: } 2-3 \text { sets } \\
\text { of } 8-12 \text { reps; Rowing: } 60-70 \% \text { to } \\
80-85 \% \text { of maximum heart rate } \\
\text { Session duration: NR for the } \\
\text { total session duration; however; } \\
\text { rowing took } 15 \text { to } 20 \text { min } \\
\text { Delivered by: NR } \\
\text { Duration of the intervention } \\
\text { (wks): } 36 \\
\text { Primary exercise type: } \\
\text { Multiple (resistance plus } \\
\text { endurance plus flexibility) }\end{array}$ & $A$ vs $B$ & $\begin{array}{l}\text { 1. Whole body } \\
\text { BMD } \\
\text { 2. Lumbar } \\
\text { spine (L2-L4) } \\
\text { BMD } \\
\text { 3. Femoral } \\
\text { neck BMD } \\
\text { 4. Trochanter } \\
\text { BMD } \\
\text { 5. Ward's BMD } \\
\text { 6. Ultra distal } \\
\text { wrist BMD } \\
\text { 7. One-third } \\
\text { distal wrist } \\
\text { BMD }\end{array}$ & 12 & $\begin{array}{l}\text { Quantitative estimates not } \\
\text { reported (chance scores } \\
\text { are provided in a graph) } \\
\text { 1. Whole body BMD } \\
\text { Positive effect towards "A" } \\
\text { 2. Lumbar spine (L2-L4) } \\
\text { BMD } \\
\text { Positive effect towards "A" } \\
\text { 3. Femoral neck BMD } \\
\text { Positive effect towards "A" } \\
\text { 4. Trochanter BMD } \\
\text { Positive effect towards "A" } \\
\text { 5. Ward's BMD } \\
\text { Positive effect towards "A" } \\
\text { 6. Ultra distal wrist BMD } \\
\text { Positive effect towards "B" } \\
\text { 7. One-third distal wrist } \\
\text { BMD } \\
\text { Positive effect towards "B" }\end{array}$ \\
\hline $\begin{array}{l}{ }^{b} \text { Rikli } 1990 \\
1 / 10[67]\end{array}$ & $\begin{array}{l}\text { Quasi- } \\
\text { randomised } \\
\text { trial } \\
37 / 31\end{array}$ & $\begin{array}{l}\text { Setting: Local } \\
\text { retirement } \\
\text { community; } \\
\text { United States } \\
\text { Health Status: } \\
\text { Healthy } \\
\text { A. General }\end{array}$ & $\begin{array}{l}\text { A. General exercise: Group- } \\
\text { based aerobic exercise training } \\
\text { for large muscle groups. } \\
\text { Frequency: } 3 \text { times/week } \\
\text { Intensity: } 60-70 \% \text { maximum } \\
\text { heart rate } \\
\text { Session duration: } 30 \text { to } 50 \text { min }\end{array}$ & $A$ vs $B$ & $\begin{array}{l}\text { 1. Distal radius } \\
\text { BMC/BW } \\
\text { 2. Distal radius } \\
\text { BMC }\end{array}$ & 10 & $\begin{array}{l}\text { Change score (\%) } \\
\text { 1. Distal radius BMC/BW } \\
\text { A. General exercise: } 0.921 \\
\text { B. General exercise and } \\
\text { weight: } 1.734 \\
\text { 2. Distal radius BMC } \\
\text { A. General exercise: } 1.023\end{array}$ \\
\hline
\end{tabular}


Table 2 Description of included studies comparing two or more forms of physical activity (Continued)

\begin{tabular}{|c|c|c|c|c|c|c|c|}
\hline $\begin{array}{l}\text { Reference } \\
\text { PEDro score }\end{array}$ & $\begin{array}{l}\text { Study } \\
\text { design } \\
\text { Allocated/ } \\
\text { Analysed }\end{array}$ & $\begin{array}{l}\text { Participants } \\
\text { (n, age mean } \\
\text { (SD), \% } \\
\text { women, } \\
\text { setting, } \\
\text { health status) }\end{array}$ & $\begin{array}{l}\text { Intervention } \\
\text { Primary exercise type } \\
\text { according to ProFANE }^{\mathrm{a}}\end{array}$ & $\begin{array}{l}\text { Relevant } \\
\text { comparison }\end{array}$ & Outcomes & $\begin{array}{l}\text { Follow } \\
\text { up } \\
(\mathrm{mo})\end{array}$ & Results \\
\hline & & $\begin{array}{l}\text { exercise } \\
\boldsymbol{n}=13 \\
\text { (randomised); } \\
10 \text { (analysed) } \\
\text { Age: } 72.2 \\
\text { (5.57) } \\
\text { B. General } \\
\text { exercise + } \\
\text { weight } \\
\boldsymbol{n}=13 \\
\text { (randomised); } \\
10 \text { (analysed) } \\
\text { Age: } 71.6 \\
\text { (5.66) } \\
\text { C. Control: } \\
\text { Not relevant } \\
\text { for this } \\
\text { comparison } \\
\text { Female: } 100 \%\end{array}$ & $\begin{array}{l}\text { Delivered by: NR } \\
\text { Duration of the intervention } \\
\text { (wks): } 40 \\
\text { Primary exercise type: } \\
\text { Endurance } \\
\text { B. General exercise + weight: } \\
\text { Group-based aerobic exercise } \\
\text { training plus upper body pro- } \\
\text { gressive resistance training. The } \\
\text { resistance training was per- } \\
\text { formed without supervision. } \\
\text { Frequency: } 3 \text { times/week } \\
\text { Intensity: } 60-70 \% \text { maximum } \\
\text { heart rate for aerobic activities } \\
\text { Session duration: } 50 \text { to } 70 \text { min } \\
\text { Delivered by: Assistants } \\
\text { Duration of the intervention } \\
\text { (wks): } 40 \\
\text { Primary exercise type: } \\
\text { Multiple (resistance plus } \\
\text { endurance) }\end{array}$ & & & & $\begin{array}{l}\text { B. General exercise and } \\
\text { weight: } 1.743 \\
\text { Statistical test not } \\
\text { performed between the } \\
\text { two intervention groups }\end{array}$ \\
\hline $\begin{array}{l}\text { Shen } 2007 \\
6 / 10[69]\end{array}$ & $\begin{array}{l}\text { RCT } \\
28 / 24\end{array}$ & $\begin{array}{l}\text { Setting: Local } \\
\text { senior living } \\
\text { campus; } \\
\text { United States } \\
\text { Health Status: } \\
\text { Healthy } \\
\text { A. Tai chi } \\
\boldsymbol{n}=14 \\
\text { (randomised); } \\
12 \text { (analysed) } \\
\text { Age: } 78.8(1.3 \text { ) } \\
\text { Female: } 79 \% \\
\text { B. Resistance } \\
\text { exercise } \\
\boldsymbol{n}=14 \\
\text { (randomised); } \\
12 \text { (analysed) } \\
\text { Age: } 79.4 \text { (2.2) } \\
\text { Female: } 71 \%\end{array}$ & $\begin{array}{l}\text { A. Tai chi: } 24 \text {-form simplified } \\
\text { Yang style Tai Chi. } \\
\text { Frequency: } 3 \text { times/week } \\
\text { Intensity: NR } \\
\text { Session duration: } 40 \text { min } \\
\text { Delivered by: Experienced Tai } \\
\text { Chi instructor } \\
\text { Duration of the intervention } \\
\text { (wks): } 24 \\
\text { Primary exercise type: } 3 D \text { (Tai } \\
\text { Chi) } \\
\text { B. Resistance exercise: Low- } \\
\text { intensity resistance training for } \\
\text { lower and upper extremities } \\
\text { using equipment and } \\
\text { dumbbells. } \\
\text { Frequency: } 3 \text { times/week } \\
\text { Intensity: } 1 \text { set of } 10-12 \text { reps at } \\
50 \% \text { of the } 1 \text { RM } \\
\text { Session duration: } 40 \text { min } \\
\text { Delivered by: Certified fitness } \\
\text { trainer } \\
\text { Duration of the intervention } \\
\text { (wks): } 24 \\
\text { Primary exercise type: } \\
\text { Resistance }\end{array}$ & $A$ vs $B$ & $\begin{array}{l}\text { 1. Bone specific } \\
\text { alkaline } \\
\text { phosphatase } \\
\text { (BAP), } \\
\text { concentration } \\
\text { change (\%) } \\
\text { 2. Pyridinoline } \\
\text { (PYD), } \\
\text { concentration } \\
\text { change (\%) } \\
\text { 3. Parathyroid } \\
\text { hormone } \\
\text { (PTH), } \\
\text { concentration } \\
\text { change (\%) }\end{array}$ & 6 & $\begin{array}{l}\text { 1. BAP } \\
\text { No between-group differ- } \\
\text { ence (positive effect to- } \\
\text { wards Tai chi) } \\
\text { 2. PYD } \\
\text { No between-group differ- } \\
\text { ence (positive effect to- } \\
\text { wards Resistance training) } \\
\text { 3. PTHP } \\
\text { No between-group differ- } \\
\text { ence (positive effect to- } \\
\text { wards Resistance training) } \\
\text { Quantitative results not } \\
\text { provided. Results reported } \\
\text { in a graph }\end{array}$ \\
\hline $\begin{array}{l}{ }^{b} \text { Woo } 2007 \\
6 / 10[75]\end{array}$ & $\begin{array}{l}\text { RCT } \\
180 / 176\end{array}$ & $\begin{array}{l}\text { Setting: } \\
\text { Community; } \\
\text { Hong Kong } \\
\text { Heath status: } \\
\text { Healthy } \\
\text { A. Tai Chi } \\
\mathbf{n}=60 \\
\text { (randomised); } \\
58 \\
\text { (randomised) } \\
\text { Age: } 68.2\end{array}$ & $\begin{array}{l}\text { A. Tai Chi: } 24 \text {-forms of Tai Chi } \\
\text { using Yang style } \\
\text { Frequency: } 3 \text { times/week } \\
\text { Intensity: NR } \\
\text { Session duration: NR } \\
\text { Delivered by: NR } \\
\text { Duration of the intervention } \\
\text { (wks): } 52 \\
\text { Primary exercise type: 3D (Tai } \\
\text { Chi) } \\
\text { B. Resistance training: }\end{array}$ & $A$ vs $B$ & $\begin{array}{l}\text { 1. Total hip } \\
\text { BMD } \\
\text { 2. Total spine } \\
\text { BMD }\end{array}$ & 12 & $\begin{array}{l}\text { Change score (mean \% } \\
\text { change } \pm \text { SE) } \\
\text { Men } \\
\text { 1. Total hip BMD } \\
\text { A. Tai Chi: }-0.48 \pm 0.37 \\
\text { B. Resistance: }-1.20 \pm 0.38 \\
\text { 2. Total spine BMD } \\
\text { A. Tai Chi: } 1.35 \pm 0.40 \\
\text { B. Resistance: } 1.27 \pm 0.42 \\
\text { Women } \\
\text { 1. Total hip BMD }\end{array}$ \\
\hline
\end{tabular}


Table 2 Description of included studies comparing two or more forms of physical activity (Continued)

\begin{tabular}{|c|c|c|c|c|c|c|c|}
\hline $\begin{array}{l}\text { Reference } \\
\text { PEDro score }\end{array}$ & $\begin{array}{l}\text { Study } \\
\text { design } \\
\text { Allocated/ } \\
\text { Analysed }\end{array}$ & $\begin{array}{l}\text { Participants } \\
\text { (n, age mean } \\
\text { (SD), \% } \\
\text { women, } \\
\text { setting, } \\
\text { health status) }\end{array}$ & $\begin{array}{l}\text { Intervention } \\
\text { Primary exercise type } \\
\text { according to ProFANE }^{\mathrm{a}}\end{array}$ & $\begin{array}{l}\text { Relevant } \\
\text { comparison }\end{array}$ & Outcomes & $\begin{array}{l}\text { Follow } \\
\text { up } \\
(\mathrm{mo})\end{array}$ & Results \\
\hline & & 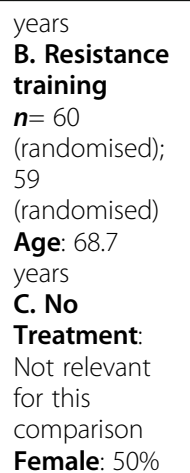 & $\begin{array}{l}\text { Resistance training with the use } \\
\text { of medium strength TheraBand } \\
\text { Frequency: } 3 \text { times/week } \\
\text { Intensity: } 30 \text { times with } \\
\text { medium strength TheraBand } \\
\text { Session duration: NR } \\
\text { Delivered by: NR } \\
\text { Duration of the intervention } \\
\text { (wks): } 52 \\
\text { Primary exercise type: } \\
\text { Resistance }\end{array}$ & & & & $\begin{array}{l}\text { A. Tai Chi: } 0.07 \pm 0.64 \\
\text { B. Resistance: } 0.09 \pm 0.62 \\
\text { 2. Total spine } B M D \\
\text { A. Tai Chi: } 0.10 \pm 0.50 \\
\text { B. Resistance: } 1.98 \pm 0.48\end{array}$ \\
\hline
\end{tabular}

$B M C$ bone mineral content $(\mathrm{g}), B M D$ bone mineral density $\left(\mathrm{g} / \mathrm{cm}^{2}\right), B M I$ body mass index $\left(\mathrm{kg} / \mathrm{m}^{2}\right), B W$ bone width, NR not reported, $R C T$ randomised controlled trial. When data was available for more than one time-point, we extracted the post-intervention and follow-up data. Mean estimates were extracted in the following hierarchical order: mean difference, change score and final score

${ }^{a}$ Exercise is a physical activity that is planned, structured and repetitive and aims to improve or maintain physical fitness. There is a wide range of possible types of exercise, and exercise programmes often include one or more types of exercise. We categorised exercise based on a modification of the Prevention of Falls Network Europe (ProFaNE) taxonomy that classifies exercise type as: i) gait, balance, and functional training; ii) strength/ resistance (including power); iii) flexibility; iv) three- dimensional (3D) exercise (e.g., Tai Chi, Qigong, dance); v) general physical activity; vi) endurance; and vii) other kind of exercises. The taxonomy allows for more than one type of exercise to be delivered within a programme. We also considered whether the exercise explicitly included bone loading eg hopping or heel drops

${ }^{\mathrm{b}}$ Indicates studies also included in the exercise vs control comparison (Table 1), but only the results for exercise comparisons are presented here

indicates articles reporting results from the same study

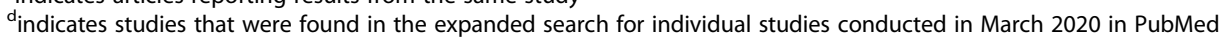

${ }$ indicates studies that were found in the updated search for systematic reviews conducted in July 2020 in PubMed, Embase, CINAHL, SPORTDiscus

* indicates statistically significant between-group differences at $p<0.05$.

related to exposure measurement, study attrition and study confounding.

\section{Association between physical activity and osteoporosis prevention}

A total of 40 articles reporting on 37 studies (30 randomised and 7 quasi-randomised trials) investigated physical activity interventions compared with a control group (Table 1). Overall the sample size for the trials was small (median: 50, range: 16 to 283) and the median follow-up length was 12 months (range 3 to 144). Meta-analysis revealed a significant but relatively small overall effect of exercise when the results of the main outcome from each study were pooled (standardised effect size 0.15 , $95 \%$ CI 0.05 to $0.25,20$ trials, Fig. 2). The quality of evidence was moderate as per GRADE system, downgraded for study limitations, meaning that the true effect is likely to be close to the estimated results (Table 5 and Additional file 5, Supplementary Table A). The overall results suggest that physical activity interventions probably improve bone health and prevent osteoporosis in older adults.

We also summarised the evidence for the two most commonly reported outcome measures across the included studies. Meta-analysis found a non-significant and small overall effect of physical activity on femoral neck BMD (standardised effect size 0.09, 95\% CI - 0.03 to $0.21,14$ trials; Fig. 3). The quality of the evidence was low, downgraded for study limitations and publication bias, suggesting limited confidence in the results (Table 5 and Additional file 5, Supplementary Table B). Overall, these results suggest that physical activity interventions may improve BMD of the femoral neck in older adults.

Lumbar spine BMD was the second mostly commonly reported outcome measures. Meta-analysis found a significant but relatively small overall effect of physical activity on lumbar spine BMD (standardised effect size $0.17,95 \%$ CI 0.04 to $0.30,11$ trials; Fig. 4 ). The quality of the evidence was moderate, downgraded for study limitations, suggesting that the true effect is likely to be close to the estimated results (Table 5 and Additional file 5, Supplementary Table C). The overall results suggest that physical activity interventions probably improve BMD of the lumbar spine in older adults.

We included 12 observational studies. Since the studies varied in terms of design, statistical approach and measures of physical activity, we did not perform metaanalysis and apply the GRADE approach. Overall, studies showed a positive effect of physical activity on bone health (Table 4). 
Table 3 Description of included studies investigating the association between different doses of physical activity on osteoporosis prevention

\begin{tabular}{|c|c|c|c|c|c|c|c|}
\hline $\begin{array}{l}\text { Reference } \\
\text { PEDro } \\
\text { score }\end{array}$ & $\begin{array}{l}\text { Study } \\
\text { design } \\
\text { Allocated/ } \\
\text { Analysed }\end{array}$ & $\begin{array}{l}\text { Participants ( } \mathrm{n} \text {, age } \\
\text { mean (SD), } \% \\
\text { women, setting, } \\
\text { health status) }\end{array}$ & $\begin{array}{l}\text { Intervention } \\
\text { Primary exercise type according to } \\
\text { ProFANE }^{\mathrm{a}}\end{array}$ & $\begin{array}{l}\text { Relevant } \\
\text { comparison }\end{array}$ & Outcomes & $\begin{array}{l}\text { Follow } \\
\text { up } \\
\text { (mo) }\end{array}$ & Results \\
\hline $\begin{array}{l}{ }^{\mathrm{b} A s h e} \\
2013 \text { [77] }\end{array}$ & $\begin{array}{l}\text { RCT } \\
155 / 147\end{array}$ & $\begin{array}{l}\text { Setting: Community; } \\
\text { Canada } \\
\text { Health status: } \\
\text { Healthy } \\
\text { A. Balance and } \\
\text { tone (BT) } \\
\text { Not relevant for this } \\
\text { comparison } \\
\text { B. Once a week } \\
\text { resistance training } \\
\text { (RT1) } \\
\boldsymbol{n}=54 \text { (randomised); } \\
47 \text { (analysed) } \\
\text { Age: } 69.4 \text { (3.0) } \\
\text { C. Twice a week } \\
\text { resistance training } \\
\text { (RT2) } \\
\text { n= } 52 \text { (randomised); } \\
\text { 46 (analysed) } \\
\text { Age: } 69.2 \text { (3.0) } \\
\text { Female: 100\% }\end{array}$ & $\begin{array}{l}\text { B. RT1: Low-frequency, group-based super- } \\
\text { vised resistance training for upper and lower } \\
\text { body with the use of resistance equipment. } \\
\text { Frequency: } 1 \text { time/week } \\
\text { Intensity: } 2 \text { sets of } 8 \text { RM } \\
\text { Session duration: NR } \\
\text { Primary exercise type: Resistance } \\
\text { C. RT2: High-frequency, group-based super- } \\
\text { vised resistance training for upper and lower } \\
\text { body with the use of resistance equipment. } \\
\text { Frequency: } 2 \text { times/week } \\
\text { Intensity: } 2 \text { sets of } 8 \text { RM } \\
\text { Primary exercise type: Resistance } \\
\text { Duration of the interventions (wks): } 52 \\
\text { Delivered by: Certified fitness instructors }\end{array}$ & B vs $C$ & $\begin{array}{l}\text { 1. Tibial } \\
\text { volumetric } \\
\text { cortical } \\
\text { density } \\
\text { (CovBMD) } \\
\text { 2. Total area } \\
\text { (ToA) } \\
\text { midtibia } \\
\text { 3. Tibial bone } \\
\text { strength }\end{array}$ & 12 & $\begin{array}{l}\text { Final score } \\
\text { (mean } \pm \text { SD) } \\
\text { 1. Tibial } \\
\text { CovBMD } \\
\text { B. }-1.81 \pm \\
-0.17 \\
\text { C. }-4.67 \pm \\
-0.45 \\
\text { 2. Total area } \\
\text { (ToA) } \\
\text { midtibia } \\
\text { B. } 0.86 \pm 0.21 \\
\text { C. } 0.93 \pm 0.22 \\
\text { 3. Tibial bone } \\
\text { strength } \\
\text { B. } 124.83 \pm \\
0.64 \\
\text { C. } 9.94 \pm 0.05\end{array}$ \\
\hline $\begin{array}{l}\text { 'Bemben } \\
2011 \\
4 / 10[91]\end{array}$ & $\begin{array}{l}\text { RCT } \\
160 / 124\end{array}$ & $\begin{array}{l}\text { Setting: Community; } \\
\text { United States } \\
\text { Health Status: } \\
\text { Healthy } \\
\text { Age: Men } 65.2 \text { (0.5); } \\
\text { Female } 63.8(0.4) \\
\text { Female: 64\% } \\
\text { A. } 2 \text { days/week } \\
\text { high intensity (2HI) } \\
\text { group } \\
\mathbf{n}=39 \text { (randomised); } \\
31 \text { (analysed) } \\
\text { B. } 2 \text { days/week low } \\
\text { intensity (2LI) } \\
\text { group } \\
\text { n=41 (randomised); } \\
34 \text { (analysed) } \\
\text { C. } 3 \text { days/week } \\
\text { high intensity (3HI) } \\
\text { group } \\
\mathbf{n}=34 \text { (randomised); } \\
24 \text { (analysed) } \\
\text { D. } 3 \text { days/week low } \\
\text { intensity (3LI) } \\
\boldsymbol{n}=46 \text { (randomised); } \\
35 \text { (analysed) }\end{array}$ & $\begin{array}{l}\text { Training included five upper body and seven } \\
\text { lower body exercise } \\
\text { A. } 2 \text { HI: } \\
\text { Frequency: } 2 \text { times/week } \\
\text { Intensity: } 80 \% \text { of } 1 \mathrm{RM}, 3 \text { sets of } 8 \text { reps } \\
\text { Session duration: } 60 \text { min } \\
\text { Delivered by: NR } \\
\text { Duration of the intervention (wks): } 40 \\
\text { Primary exercise type: Resistance } \\
\text { B. } 2 \text { Ll: } \\
\text { Frequency: } 2 \text { times/week } \\
\text { Intensity: } 40 \% \text { of } 1 \text { RM, } 3 \text { sets of } 16 \text { reps } \\
\text { Session duration: } 60 \text { min } \\
\text { Delivered by: NR } \\
\text { Duration of the intervention (wks): } 40 \\
\text { Primary exercise type: Resistance } \\
\text { C. } 3 \text { HI: } \\
\text { Frequency: } 3 \text { times/week } \\
\text { Intensity: } 80 \% \text { of } 1 \text { RM, } 3 \text { sets of } 8 \text { reps } \\
\text { Session duration: } 60 \text { min } \\
\text { Delivered by: NR } \\
\text { Duration of the intervention (wks): } 40 \\
\text { Primary exercise type: Resistance } \\
\text { D. } 3 \text { LI: } \\
\text { Frequency: } 3 \text { times/week } \\
\text { Intensity: } 40 \% \text { of } 1 \text { RM, } 3 \text { sets of } 16 \text { reps } \\
\text { Session duration: } 60 \text { min } \\
\text { Delivered by: NR } \\
\text { Duration of the intervention (wks): } 40 \\
\text { Primary exercise type: Resistance }\end{array}$ & $\begin{array}{l}\text { A vs } B \\
A \text { vs } C \\
C \text { vs } D \\
B \text { vs } D\end{array}$ & $\begin{array}{l}\text { 1. Lumbar } \\
\text { spine (L2-4) } \\
\text { BMD } \\
\text { 2. Femoral } \\
\text { neck BMD } \\
\text { 3. Trochanter } \\
\text { BMD } \\
\text { 4. Total hip } \\
\text { BMD } \\
\text { 5. Total body } \\
\text { BMD }\end{array}$ & 10 & 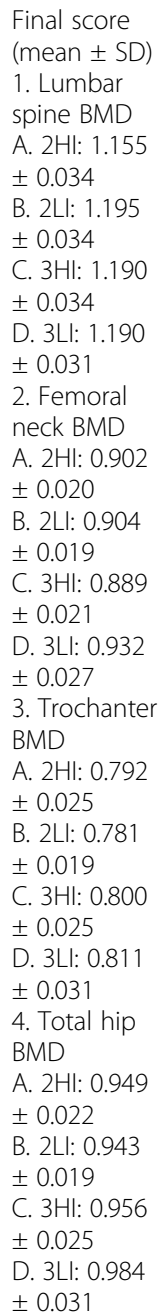 \\
\hline
\end{tabular}


Table 3 Description of included studies investigating the association between different doses of physical activity on osteoporosis prevention (Continued)

\begin{tabular}{|c|c|c|c|c|c|c|c|}
\hline $\begin{array}{l}\text { Reference } \\
\text { PEDro } \\
\text { score }\end{array}$ & $\begin{array}{l}\text { Study } \\
\text { design } \\
\text { Allocated/ } \\
\text { Analysed }\end{array}$ & $\begin{array}{l}\text { Participants ( } \mathrm{n} \text {, age } \\
\text { mean (SD), \% } \\
\text { women, setting, } \\
\text { health status) }\end{array}$ & $\begin{array}{l}\text { Intervention } \\
\text { Primary exercise type according to } \\
\text { ProFANE }^{\mathrm{a}}\end{array}$ & $\begin{array}{l}\text { Relevant } \\
\text { comparison }\end{array}$ & Outcomes & $\begin{array}{l}\text { Follow } \\
\text { up } \\
\text { (mo) }\end{array}$ & Results \\
\hline & & & & & & & $\begin{array}{l}\text { 5. Total body } \\
\text { BMD } \\
\text { A. } 2 \mathrm{HI}: 1.172 \\
\pm 0.014 \\
\text { B. } 2 \text { Ll: } 1.175 \\
\pm 0.015 \\
\text { C. } 3 \mathrm{HI}: 1.199 \\
\pm 0.020 \\
\text { D. } 3 \text { Ll: } 1.177 \\
\pm 0.017^{*}\end{array}$ \\
\hline $\begin{array}{l}\text { Kemmler } \\
2010 \\
6 / 10[54]\end{array}$ & $\begin{array}{l}\text { RCT } \\
246 / 227\end{array}$ & $\begin{array}{l}\text { Setting: Community; } \\
\text { Germany } \\
\text { Health status: } \\
\text { Healthy } \\
\text { A. Multi- } \\
\text { component exer- } \\
\text { cise training } \\
\boldsymbol{n}=123 \\
\text { (randomised); } 115 \\
\text { (analysed) } \\
\text { Age: } 68.9 \text { (3.9) } \\
\text { B. Low intensity }\end{array}$ & $\begin{array}{l}\text { A. Two 60-minute supervised group sessions: } \\
\text { warm-up/ aerobic dance ( } 20 \text { min), balance } \\
\text { training ( } 5 \text { min); functional gymnastics, iso- } \\
\text { metric strength training with } 1-3 \text { sets of iso- } \\
\text { metric floor exercises for trunk flexors and } \\
\text { extensors hip flexors and extensors and leg } \\
\text { abductors and adductors; upper body } \\
\text { exercises. } \\
\text { Two home training session that includes } \\
\text { strength and flexibility training. } \\
\text { Frequency: } 4 \text { sessions/week } \\
\text { Intensity: Aerobic dance: } 70 \%-85 \% \text { of }\end{array}$ & $A$ vs $B$ & $\begin{array}{l}\text { 1. Lumbar } \\
\text { spine BMD } \\
\text { 2. Femoral } \\
\text { neck BMD }\end{array}$ & 18 & $\begin{array}{l}\text { Mean } \\
\text { difference } \\
\text { (95\% Cl) } \\
\text { 1. Lumbar } \\
\text { spine BMD: } \\
0.014(0.006 \\
\text { to } 0.021) \\
\text { 2. Femoral } \\
\text { neck BMD: } \\
0.015(0.008 \\
\text { to } 0.021)^{*}\end{array}$ \\
\hline
\end{tabular}

$\begin{array}{ll}{ }^{\text {bPruitt }} & \text { RCT } \\ 1995 & 40 / 26 \\ 4 / 10[64] & \end{array}$

B. Low intensity multicomponent programme $\boldsymbol{n}=123$ (randomised); 112 (analysed) Age: 69.2 (4.1) Female: $100 \%$
Intensity: Aerobic dance: $70 \%-85 \%$ of

maximum heart rate; Upper body exercise: 10-15 reps $\times$ 2-3sets;

Home training session: $1-2$ sets of 6-8 isometric exercise and 10-15 reps $\times 2$ sets of belt exercises

Session duration: $60 \mathrm{~min} /$ group class \& 20

min/home training session

Delivered by: Certified trainer

Duration of the intervention (wks): 72

Primary exercise type: Multiple (balance

and function plus resistance)

B. Low intensity multicomponent programme including walking, muscular relaxation, endurance and strength training

Frequency: 1 session/week

Intensity: Walking at 50-60\% maximum heart rate

Endurance and strength training: low to moderate intensity

Session duration: $60 \mathrm{~min}$

Delivered by: Certified trainer

Duration of the intervention (wks): 72

[every 10 weeks of training was followed by

10 weeks of rest]

Primary exercise type: Multiple (balance

and function plus endurance)

Setting: Community; America

Healthy status

Healthy

A. High intensity resistance training

$\boldsymbol{n}=15$ (randomised); 8 (analysed)

Age: $67.0(0.5)$

B. Low intensity resistance training

$\boldsymbol{n}=13$ (randomised); 7 (analysed)

Age: 67.6 (1.4)

C. Control: not
A and B. Supervised exercise session comprising bench press, lateral pull down, military press, biceps curl, knee extension, knee flexion, hip abduction and adduction, leg press, back extension.

A. Intensity: High

14 reps $\times 1$ set at 40\% 1RM for warm up; 7 reps $\times 2$ sets at $80 \% 1 \mathrm{RM}$

B. Intensity: Low

14 reps $\times 3$ sets at 40\% 1RM

For both $A$ and $B$ :

Frequency: 3 times/week

Session duration: $50-55 \mathrm{~min} /$ lifting time Delivered by: NR

Duration of the intervention (wks): 52

$\begin{array}{lll}\text { A vs B } & \text { 1. Total hip } 12 & \text { Change } \\ \text { BMD } & \text { score (mean } \\ \text { 2. Femoral } & \pm \text { SD) } \\ \text { neck BMD } & \text { 1. Total hip } \\ \text { 3. Ward's } & \text { BMD } \\ \text { triangle BMD } & \text { A. High } \\ \text { 4. Lumbar } & \text { intensity: } \\ \text { spine (L2-L4) } & 0.005 \pm 0.014 \\ \text { BMD } & \text { B. Low } \\ & \text { intensity: } \\ & 0.008 \pm 0.012 \\ & \text { 2. Femoral } \\ & \text { neck BMD } \\ & \text { A. High } \\ & \text { intensity: }\end{array}$


Table 3 Description of included studies investigating the association between different doses of physical activity on osteoporosis prevention (Continued)

\begin{tabular}{|c|c|c|c|c|c|c|c|}
\hline $\begin{array}{l}\text { Reference } \\
\text { PEDro } \\
\text { score }\end{array}$ & $\begin{array}{l}\text { Study } \\
\text { design } \\
\text { Allocated/ } \\
\text { Analysed }\end{array}$ & $\begin{array}{l}\text { Participants ( } \mathrm{n} \text {, age } \\
\text { mean (SD), } \% \\
\text { women, setting, } \\
\text { health status) }\end{array}$ & $\begin{array}{l}\text { Intervention } \\
\text { Primary exercise type according to }_{\text {ProFANE }^{\mathrm{a}}}\end{array}$ & $\begin{array}{l}\text { Relevant } \\
\text { comparison }\end{array}$ & Outcomes & $\begin{array}{l}\text { Follow } \\
\text { up } \\
\text { (mo) }\end{array}$ & Results \\
\hline & & $\begin{array}{l}\text { relevant for this } \\
\text { comparison } \\
\text { Female: } 100 \%\end{array}$ & & & & & $\begin{array}{l}-0.002 \pm \\
0.154 \\
\text { B. Low } \\
\text { intensity: } \\
0.025 \pm 0.008 \\
\text { 3. Ward's } \\
\text { triangle BMD } \\
\text { A. High } \\
\text { intensity: } \\
0.018 \pm 0.032 \\
\text { B. Low } \\
\text { intensity: } \\
0.022 \pm 0.045 \\
\text { 4. Lumbar } \\
\text { spine (L2-L4) } \\
\text { BMD } \\
\text { A. High } \\
\text { intensity: } \\
0.007 \pm 0.018 \\
\text { B. Low } \\
\text { intensity: } \\
0.005 \pm 0.027\end{array}$ \\
\hline $\begin{array}{l}{ }^{\mathrm{d}} \text { Taaffe } \\
1996 \\
4 / 10[101]\end{array}$ & $\begin{array}{l}\text { RCT } \\
36 / 21\end{array}$ & $\begin{array}{l}\text { Setting: Community; } \\
\text { United States } \\
\text { Health status: } \\
\text { Healthy } \\
\text { A. High intensity } \\
\text { resistance training } \\
\text { group } \\
\boldsymbol{n}=12 \text { (randomised); } \\
7 \text { (analysed) } \\
\text { Age: } 67.0 \text { (0.2) } \\
\text { B. Low intensity } \\
\text { resistance training } \\
\text { group } \\
\boldsymbol{n}=13 \text { (randomised); } \\
7 \text { (analysed) } \\
\text { Age: } 67.6(0.5) \\
\text { C. Control } \\
\boldsymbol{n}=11 \text { (randomised); } \\
7 \text { (analysed) } \\
\text { Age: } 69.6 \text { (1.3) } \\
\text { Female: } 100 \%\end{array}$ & $\begin{array}{l}\text { Supervised exercise training targeted thigh } \\
\text { muscle strength including leg press, knee } \\
\text { extension and knee flexion. Exercise sessions } \\
\text { were bracketed by warm up and cool-down } \\
\text { periods } \\
\text { A. Intensity: } 1 \text { set of } 14 \text { reps at an intensity } \\
\text { of } 40 \% 1 \text { RM and } 2 \text { sets of } 7 \text { reps at an } \\
\text { intensity of } 80 \% \text { of } 1 \text { RM } \\
\text { B. Intensity: } 3 \text { sets of } 14 \text { reps at an intensity } \\
\text { of } 40 \% \text { of } 1 \text { RM } \\
\text { For both groups } \\
\text { Frequency: } 3 \text { times/week } \\
\text { Session duration: NR } \\
\text { Delivered by: NR } \\
\text { Duration of the intervention (wks): } 52 \\
\text { Primary exercise type: Resistance }\end{array}$ & $\begin{array}{l}\text { A vs } B \\
A \text { vs } C \\
B \text { vs } C\end{array}$ & $\begin{array}{l}\text { 1. Middle } \\
\text { third of the } \\
\text { femur BMD } \\
\text { 2. Thigh BMD }\end{array}$ & 12 & $\begin{array}{l}\% \text { Change } \\
\text { score (mean } \\
\pm \text { SEM) } \\
\text { 1. Middle } \\
\text { third of the } \\
\text { femur BMDF } \\
\text { A. High } \\
\text { intensity: } \\
1.0 \pm 1.0 \\
\text { B. Low } \\
\text { inteisty: -2.2 } \\
\pm 0.5 \\
\text { C. Control: } \\
-1.8 \pm 0.6 \\
\text { 2. NR }\end{array}$ \\
\hline $\begin{array}{l}{ }^{\text {b}} \text { Taaffe } \\
1999 \\
5 / 10[72]\end{array}$ & $\begin{array}{l}\text { RCT } \\
53 / 46\end{array}$ & $\begin{array}{l}\text { Setting: Community; } \\
\text { United States } \\
\text { Health status: } \\
\text { Healthy } \\
\text { A. High-intensity } \\
\text { resistance training } \\
\text { (1 day per week) } \\
\boldsymbol{n}=14 \text { (randomised); } \\
11 \text { (analysed) } \\
\text { Age: } 68.5 \text { (3.6) } \\
\text { Female: } 36 \% \\
\text { B. High-intensity } \\
\text { resistance training } \\
\text { (2 days per week) } \\
\boldsymbol{n}=14 \text { (randomised); } \\
12 \text { (analysed) } \\
\text { Age: } 69.4 \text { (3.0) } \\
\text { Female: } 29 \% \\
\text { C. High-intensity } \\
\text { resistance training }\end{array}$ & $\begin{array}{l}\text { Training includes the whole body (bench } \\
\text { press, military press, latissimus pull-down, bi- } \\
\text { ceps curl, and leg press) } \\
\text { All trainings were started with a warm up } \\
\text { that included stretching and one set each of } \\
\text { bench press and leg press ( } 40 \% \text { of } 1-R M, 10 \\
\text { repetitions) and concluded with a cool-down } \\
\text { period of stretching. } \\
\text { Intensity: started at } 60 \% \text { of the } 1 \text { RM and } \\
\text { gradually increase in intensity } \\
\text { A. Frequency: } 1 \text { time/week } \\
\text { B. Frequency: } 2 \text { times/week } \\
\text { C. Frequency: } 3 \text { times/week } \\
\text { Intensity: } 8 \text { reps } \times 3 \text { sets at } 80 \% \text { of } 1 \text { RM } \\
\text { Session duration: NR } \\
\text { Delivered by: NR } \\
\text { Duration of the intervention (wks): } 24\end{array}$ & $\begin{array}{l}\text { A vs } B \\
\text { A vs } C \\
B \text { vs } C\end{array}$ & $\begin{array}{l}\text { 1. Lumbar } \\
\text { spine (L2-L4) } \\
\text { BMD } \\
\text { 2. Total hip } \\
\text { BMD } \\
\text { 3. Midradius } \\
\text { BMD } \\
\text { 4. Total body } \\
\text { BMC }\end{array}$ & 6 & $\begin{array}{l}\text { Final score } \\
\text { (mean } \pm \text { SD) } \\
\text { 1. Lumbar } \\
\text { spine (L2-L4) } \\
\text { BMD } \\
\text { A. Resistance } \\
1 \times / \text { week: } \\
1.025 \pm 0.006 \\
\text { B. Resistance } \\
\text { 2x/week: } \\
\text { 1.033 } \pm 0.006 \\
\text { C. Resistance } \\
\text { 3x/week: } \\
\text { 1.032 } \pm 0.007 \\
\text { 2. Total hip } \\
\text { BMD } \\
\text { A. Resistance } \\
\text { 1x/week: } \\
0.865 \pm 0.010 \\
\text { B. Resistance }\end{array}$ \\
\hline
\end{tabular}


Table 3 Description of included studies investigating the association between different doses of physical activity on osteoporosis prevention (Continued)

\begin{tabular}{|c|c|c|c|c|c|c|c|}
\hline $\begin{array}{l}\text { Reference } \\
\text { PEDro } \\
\text { score }\end{array}$ & $\begin{array}{l}\text { Study } \\
\text { design } \\
\text { Allocated/ } \\
\text { Analysed }\end{array}$ & $\begin{array}{l}\text { Participants (n, age } \\
\text { mean (SD), \% } \\
\text { women, setting, } \\
\text { health status) }\end{array}$ & $\begin{array}{l}\text { Intervention } \\
\text { Primary exercise type according to } \\
\text { ProFANE }^{\mathrm{a}}\end{array}$ & $\begin{array}{l}\text { Relevant } \\
\text { comparison }\end{array}$ & Outcomes & $\begin{array}{l}\text { Follow } \\
\text { up } \\
\text { (mo) }\end{array}$ & Results \\
\hline & & $\begin{array}{l}\text { ( } 3 \text { days per week) } \\
\boldsymbol{n}=11 \text { (randomised } \\
\& \text { analysed) } \\
\text { Age: } 71.0(4.1) \\
\text { Female: } 36 \% \\
\text { D. Control: not } \\
\text { relevant for this } \\
\text { comparison }\end{array}$ & & & & & $\begin{array}{l}\text { 2x/week: } \\
0.866 \pm 0.006 \\
\text { C. Resistance } \\
3 \times / \text { week: } \\
0.864 \pm 0.010 \\
\text { 3. Midradius } \\
\text { BMD } \\
\text { A. Resistance } \\
\text { 1x/week: } \\
0.605 \pm 0.003 \\
\text { B. Resistance } \\
\text { 2x/week: } \\
0.604 \pm 0.003 \\
\text { C. Resistance } \\
3 x / \text { week: } \\
0.608 \pm 0.003 \\
\text { 4. Total body } \\
\text { BMC } \\
\text { A. Resistance } \\
\text { 1x/week: } \\
\text { 2552 } \pm 15 \\
\text { B. Resistance } \\
\text { 2x/week: } \\
\text { 2530 } 14 \\
\text { C. Resistance } \\
\text { 3x/week: } \\
\text { 2525 } \pm 14\end{array}$ \\
\hline
\end{tabular}

BMC: bone mineral content (g); BMD: bone mineral density $\left(\mathrm{g} / \mathrm{cm}^{2}\right)$; BMI: body mass index $\left(\mathrm{kg} / \mathrm{m}^{2}\right)$; NR: not reported; RCT: randomised controlled trial. When data was available for more than one time-point, we extracted the post-intervention data. Mean estimates were extracted in the following hierarchical order: mean difference, change score and final score

${ }^{a}$ Exercise is a physical activity that is planned, structured and repetitive and aims to improve or maintain physical fitness. There is a wide range of possible types of exercise, and exercise programmes often include one or more types of exercise. We categorised exercise based on a modification of the Prevention of Falls Network Europe (ProFaNE) taxonomy that classifies exercise type as: i) gait, balance, and functional training; ii) strength/ resistance (including power); iii) flexibility; iv) three- dimensional (3D) exercise (e.g., Tai Chi, Qigong, dance); v) general physical activity; vi) endurance; and vii) other kind of exercises. The taxonomy allows for more than one type of exercise to be delivered within a programme. We also considered whether the exercise explicitly included bone loading eg hopping or heel drops

${ }^{b}$ Indicate studies also included in the exercise vs control comparison (Table 1) or in the one or more forms of physical activity comparison (Table 2), but only the results for different doses of exercise are presented here

indicates studies that were found in the expanded search for individual studies conducted in March 2020 in PubMed

${ }^{d}$ indicates studies that were found in the updated search for systematic reviews conducted in July 2020 in PubMed, Embase, CINAHL, SPORTDiscus

*indicates statistically significant between-group difference at $p<0.05$

\section{Dose response association}

As shown in Table 1, programs which had significant impacts were generally of a higher dose. Typical program for which significant intervention impacts were detected in randomised controlled trials were undertaken for $60+$ mins, 2-3 times/week for $7+$ months $[45,52,59,63,71]$. The randomised controlled trials $(n=6)$ investigating different doses of physical activity on bone health did not suggest a clear dose-response relationship (Table 3) but were probably too small (i.e., lacked statistical power) to detect differences between different doses of physical activity. All eight longitudinal studies investigating different doses of total or planned physical activity on bone health found that higher levels of physical activity were associated with better bone health (Table 4).
Meta-regression revealed a non-significant trend for studies with a higher overall intervention dose (i.e., $7800+$ total mins) to have greater effects on femoral neck BMD $(p=0.144)$, where high dose interventions $(7800+$ mins) had a moderate effect with a standardised effect size of $0.26,95 \%$ CI -0.01 to 0.52 and lower dose interventions (<7800 mins) had a small effect $0.03,95 \%$ CI -0.12 to 0.19 , although neither sub-group effect was statistically significant. Similar results were found for lumbar spine BMD, where the difference in effects did not reach statistical significance $(p=0.373)$, but higher dose interventions had a moderate effect (standardised effect $0.33,95 \%$ CI -0.08 to 0.73 ) whereas lower dose interventions had a small effect (standardised effect 0.14, $95 \%$ CI -0.02 to 0.30 ), although neither sub-group effect was statistically significant. 
Table 4 Description of included studies investigating the association between physical activity and osteoporosis employing an observational design

\begin{tabular}{|c|c|c|c|c|c|}
\hline $\begin{array}{l}\text { Reference } \\
\text { Overall Risk of } \\
\text { Bias }\end{array}$ & $\begin{array}{l}\text { Study design } \\
\text { Included / Analysed }\end{array}$ & $\begin{array}{l}\text { Participants (n, } \\
\text { age mean (SD), } \\
\% \text { women, } \\
\text { setting, health } \\
\text { status) }\end{array}$ & Exposure & Outcomes & Results \\
\hline $\begin{array}{l}\text { aFoley } 2010 \\
\text { (Tasmania Older } \\
\text { Adult Cohort } \\
\text { study) } \\
\text { Overall risk of } \\
\text { bias: High [81] }\end{array}$ & $\begin{array}{l}\text { Prospective cohort } \\
\text { study } \\
\text { ( } 2.6 \text { years follow-up) } \\
875\end{array}$ & $\begin{array}{l}\text { Setting: } \\
\text { Community, } \\
\text { Australia } \\
\text { Health status: } \\
\text { Healthy } \\
\boldsymbol{n}=875 \text { (included) } \\
\text { Age: } 62.7 \text { (7.3) } \\
\text { (included) } \\
\text { [NB: quartile 1, } 2 \\
\text { and } 3 \text { are not of } \\
\text { interest in this } \\
\text { study as mid- } \\
\text { point age <65 } \\
\text { years] } \\
\text { Female: 49\% } \\
\text { Mid-point of } \\
\text { age quartile 4: } \\
\text { Age: } 74.8\end{array}$ & $\begin{array}{l}\text { Ambulatory activity (steps per } \\
\text { day) was assessed using } \\
\text { pedometer for } 1 \text { week at both } \\
\text { baseline and follow-up and par- } \\
\text { ticipants were divided in } \\
\text { quartiles } \\
\text { Classification: total physical } \\
\text { activity }\end{array}$ & $\begin{array}{l}\text { 1. Lumbar spine areal } \\
\text { BMD } \\
\text { 2. Hip areal BMD }\end{array}$ & $\begin{array}{l}\text { Adjusted point estimates } \\
\text { (95\% Cls) } \\
\text { 1. Lumbar spine areal } \\
\text { BMD } \\
\text { Not reported for sample } \\
\text { >65 years } \\
\text { 2. Hip areal BMD at } \\
\text { follow-up } \\
\text { Age quartile 4, Female } \\
\text { Q1. } 0.434(0.372 \text { to } 0.497) \\
\text { Q2. } 0.441(0.378 \text { to } 0.503) \\
\text { Q3. } 0.446(0.383 \text { to } 0.509) \\
\text { Q4. } 0.466(0.401 \text { to } 0.532) \\
\text { Age, quartile } 4 \text {, Male } \\
\text { Q1. } 0.554(0.490 \text { to } 0.618) \\
\text { Q2. } 0.566(0.501 \text { to } 0.631) \\
\text { Q3. } 0.572(0.507 \text { to } 0.637) \\
\text { Q4. } 0.584(0.518 \text { to } 0.651)\end{array}$ \\
\hline $\begin{array}{l}{ }^{a} \text { Muir } 2013 \\
\text { (Canadian } \\
\text { multicentre } \\
\text { osteoporosis } \\
\text { study) } \\
\text { Overall risk of } \\
\text { bias: Low [84] }\end{array}$ & $\begin{array}{l}\text { Retrospective (previous } \\
12 \text { months) }\end{array}$ & $\begin{array}{l}\text { Setting: } \\
\text { Community; } \\
\text { Canada } \\
\text { Health status: } \\
\text { Mixed (included } \\
\text { participants with } \\
\text { diagnosis of } \\
\text { osteoporosis) } \\
\boldsymbol{n}=1169 \\
\text { Age: } 79.84 \text { (4.43) } \\
\text { Female: } 100 \%\end{array}$ & $\begin{array}{l}\text { Physical activity was quantified } \\
\text { based on the level of activity } \\
\text { (moderate or strenuous or } \\
\text { vigorous) and the reported } \\
\text { frequency and duration of said } \\
\text { activity over the course of the } \\
\text { previous } 12 \text { months. } \\
\text { Classification: total physical } \\
\text { activity }\end{array}$ & $\begin{array}{l}\text { 1. Lumbar Spine (L1-4) } \\
\text { BMD } \\
\text { 2. Femoral neck BMD } \\
\text { 3. Total hip BMD } \\
\text { 4. Ward's triangle BMD } \\
\text { 5. Trochanter BMD }\end{array}$ & $\begin{array}{l}\text { Multiple regression } \\
\text { analysis of the relative } \\
\text { effects of moderate } \\
\text { activity on BMD. } \\
\text { Coefficient }(95 \% \mathrm{Cl}) \\
\text { 1. Lumbar Spine }(\mathrm{L} 1-4) \\
\text { BMD } \\
\text {-0.006 (-0.013 to } 0.000) \\
\text { 2. Femoral neck BMD } \\
\text { 0.004 }(0.000 \text { to } 0.008)^{¥} \\
\text { 3. Total hip BMD } \\
\text { 0.006 }(0.001 \text { to } 0.011)^{*} \\
\text { 4. Ward's triangle } \mathrm{BMD} \\
\text { 0.004 }(-0.001 \text { to } 0.009) \\
\text { 5. Trochanter BMD } \\
\text { 0.005 }(0.006 \text { to } 0.074)^{*}\end{array}$ \\
\hline $\begin{array}{l}{ }^{a} \text { Nakamura } 2012 \\
\text { (Muramatsu } \\
\text { Study) } \\
\text { Overall risk of } \\
\text { bias: High [85] }\end{array}$ & $\begin{array}{l}\text { Cohort } \\
\text { (6 years follow-up) } \\
774 / 382\end{array}$ & $\begin{array}{l}\text { Setting: } \\
\text { Community; } \\
\text { Japan } \\
\text { Health status: } \\
\text { Healthy } \\
\boldsymbol{n}=382 \\
\text { Age: } 73.3(3.7) \\
\text { Female: } 100 \%\end{array}$ & $\begin{array}{l}\text { Physical activity was assessed } \\
\text { via questionnaire based on } \\
\text { whether participants regularly } \\
\text { engaged in light or moderate } \\
\text { physical activity (yes/no) } \\
\text { activities: } \\
\text { A. Light physical activity } \\
\text { (includes croquet, taking walks } \\
\text { and traditional Japanese } \\
\text { dancing): yes or no }\end{array}$ & 1. Forearm BMD & $\begin{array}{l}\text { p-value association } \\
\text { between baseline } \\
\text { physical activity levels } \\
\text { BMD changes } \\
\text { 1. Forearm BMD } \\
\text { A. Light: } p=0.5122 \text { or } \\
\text { B. Moderate: } p=0.0711 \\
\text { [Quantitative estimates } \\
\text { not reported] }\end{array}$ \\
\hline
\end{tabular}

\section{dancing): yes or no}

B. Moderate physical activity (includes farm work and gardening): yes or no

Classification: total physical activity (light and moderate)

$\begin{array}{lll}{ }^{\text {RRodriguez- }} & \text { Cohort } & \text { Setting: } \\ \text { Gomez 2019 } & \text { (4 years follow-up) } & \text { Community; } \\ \text { (Toledo Study for } & 227 / 192 & \text { United States } \\ \text { Healthy Aging) } & & \text { Health status: } \\ \text { Overall risk of } & & \text { Healthy } \\ \text { bias: High [86] } & & \boldsymbol{n}=192 \\ & & \text { Age: } 80.5(4.3) \\ & & \text { Female: } \mathbf{5 2 . 6 \%}\end{array}$

Physical activity was assessed by accelerometry during waking hours for seven consecutive days, except while bathing or swimming activities A. Sedentary behaviours mean \% of waking hours: 55 (baseline); 59 (follow-up) B. Light physical activity mean \% of waking hours: 41.9 (baseline); 38.8 (follow-up) C. Moderate to vigorous
1. Whole body BMC 2. Pelvic BMC

3. Arms (mean) BMC 4. Legs (mean) BMC 5. Lumbar spine (L1-4) BMC

6. Femoral regions

BMC (proximal femur - mean, femoral neck trochanter, ward's triangle)

7. Whole body BMD
NB: Only significant findings were reported here]

Multiple regression coefficient (y) of change in the composition of movement behaviours and changes in BMC or BMD:

4. Legs (mean) BMC

A. NS

B. NS 
Table 4 Description of included studies investigating the association between physical activity and osteoporosis employing an observational design (Continued)

\begin{tabular}{lllll}
\hline $\begin{array}{l}\text { Reference } \\
\text { Overall Risk of } \\
\text { Bias }\end{array}$ & $\begin{array}{l}\text { Study design } \\
\text { Included / Analysed }\end{array}$ & $\begin{array}{l}\text { Participants (n, Exposure } \\
\text { age mean (SD), } \\
\text { \% women, } \\
\text { setting, health } \\
\text { status) }\end{array}$ & Outcomes & Results \\
\hline
\end{tabular}

\begin{tabular}{|c|c|c|c|c|c|}
\hline & & & $\begin{array}{l}\text { physical activity - mean \% of } \\
\text { waking hours: } 3.1 \text { (baseline); } 2.2 \\
\text { (follow-up) } \\
\text { Classification: total physical } \\
\text { activity }\end{array}$ & $\begin{array}{l}\text { 8. Pelvic BMD } \\
\text { 9. Arms (mean) } \\
\text { BMD10. Legs (mean) } \\
\text { BMD } \\
\text { 11. Lumbar spine (L1- } \\
\text { 4) BMD } \\
\text { 12. Femoral regions } \\
\text { BMD (proximal femur } \\
\text { - mean, femoral neck, } \\
\text { trochanter, ward's } \\
\text { triangle) }\end{array}$ & $\begin{array}{l}\text { C. } y=1.767, p=0.04^{¥} \\
\text { 5. Lumbar spine }(\mathrm{L} 1-4) \\
\text { BMC } \\
\text { A. NS } \\
\text { B. NS } \\
\text { C. } y=0.050, p=0.03^{¥} \\
\text { 10. Legs (mean) BMD } \\
\text { A. NS } \\
\text { B. NS } \\
\text { C. } y=0.005, p=0.04^{¥}\end{array}$ \\
\hline $\begin{array}{l}\text { aShephard } 2017 \\
\text { (Nakanojo Study) } \\
\text { Overall risk of } \\
\text { bias: Low [87] }\end{array}$ & $\begin{array}{l}\text { Cohort } \\
\text { (5 years follow-up) } \\
615 / 496\end{array}$ & $\begin{array}{l}\text { Setting: } \\
\text { Community; } \\
\text { Japan } \\
\text { Health status: } \\
\text { Healthy } \\
\text { Men } \\
\text { Age: } 71.2 \text { (3.9) } \\
\boldsymbol{n}=212 \\
\text { Women } \\
\text { Age: } 71.3 \text { (4.2) } \\
\boldsymbol{n}=284 \\
\text { Female: } 57.3 \%\end{array}$ & $\begin{array}{l}\text { Physical activity was measured } \\
\text { using pedometer for } 5 \text { years } \\
\text { and analysed as daily step } \\
\text { count and the daily duration of } \\
\text { exercise at an intensity }>3 \text { METs } \\
\text { A. Physical activity level } \\
\text { Quartile } 1 \\
\text { Step count (steps/day) at } \\
\text { baseline: } 3888 \text { (1117) [men]; } \\
\text { 3824 (1298) [women] } \\
\text { Duration of activity > } 3 \text { METs } \\
\text { (min/day): } 4.0 \text { (1.8) [men]; } 4.0 \\
\text { (2.4) [women] } \\
\text { B. Physical activity level } \\
\text { Quartile } 2 \\
\text { Step count (steps/day) at } \\
\text { baseline: } 5994 \text { (943) [men]; } \\
\text { 5931 (924) [women] } \\
\text { Duration of activity > } 3 \text { METs } \\
\text { (min/day): } 10.9 \text { (2.4) [men]; } 10.1 \\
\text { (2.3) [women] } \\
\text { C. Physical activity level } \\
\text { Quartile } 3 \\
\text { Step count (steps/day) at } \\
\text { baseline: } 7521 \text { (833) [men]; } \\
7626 \text { (691) [women] } \\
\text { Duration of activity > } 3 \text { METs } \\
\text { (min/day): } 19.3 \text { (2.9) [men]; } 18.4 \\
\text { (2.6) [women] } \\
\text { D. Physical activity level } \\
\text { Quartile } 4 \\
\text { Step count (steps/day) at } \\
\text { baseline: } 10892 \text { (1433) [men]; } \\
\text { 10199 (1398) [women] } \\
\text { Duration of activity }>3 \text { METs } \\
\text { (min/day): } 31.8 \text { (5.6) [men]; } 30.3 \\
\text { (4.3) [women] } \\
\text { Classification: total physical } \\
\text { activity }\end{array}$ & $\begin{array}{l}\text { 1. Osteosonic Index } \\
\text { (OSI) from the } \\
\text { ultrasonic } \\
\text { measurement of the } \\
\text { calcaneus (Fracture } \\
\text { threshold - yes/no) }\end{array}$ & $\begin{array}{l}\text { Multivariate Cox } \\
\text { proportional hazard ratio } \\
\text { (risk of the OSI falling } \\
\text { below the fracture } \\
\text { threshold) and } 95 \% \mathrm{Cl} \\
\text { Step count (steps/day) } \\
\text { Men } \\
\text { Q1. } 2.63(1.35 \text { to } 4.41)^{*} \\
\text { Q2. } 1.75(1.03 \text { to } 3.95)^{*} \\
\text { Q3. } 1.01 \text { (0.55 to } 3.37) \\
\text { Q4. } 1 \\
\text { Women } \\
\text { Q1. } 3.33(2.10 \text { to } 5.21)^{*} \\
\text { Q2. } 2.51(1.25 \text { to } 4.03)^{*} \\
\text { Q3. } 1.12(0.47 \text { to } 2.16) \\
\text { Q4. } 1 \\
\text { Duration of activity }>3 \\
\text { METs (min/day) } \\
\text { Men } \\
\text { Q1. } 2.77(1.46 \text { to } 5.59)^{*} \\
\text { Q2. } 1.91(1.02 \text { to } 3.99)^{*} \\
\text { Q3. } 1.00(0.48 \text { to } 2.27) \\
\text { Q4. } 1 \\
\text { Women } \\
\text { Q1. } 3.94(2.35 \text { to } 6.73)^{*} \\
\text { Q2. } 1.87(1.00 \text { to } 3.60)^{*} \\
\text { Q3. } 0.99 \text { (0.40 to 2.06) } \\
\text { Q4. } 1\end{array}$ \\
\hline $\begin{array}{l}{ }^{\text {aSvejme }} 2014 \\
\text { Overall risk of } \\
\text { bias: Low [88] }\end{array}$ & $\begin{array}{l}\text { Cohort } \\
\text { (25 years follow-up) }\end{array}$ & $\begin{array}{l}\text { Setting: } \\
\text { Community; } \\
\text { Sweden } \\
\text { Health status: } \\
\text { Healthy } \\
\text { A. Active } \\
\text { women } \\
\boldsymbol{n}=91 \\
\text { B. Inactive } \\
\text { women } \\
\boldsymbol{n}=21 \\
\text { Age: women }\end{array}$ & $\begin{array}{l}\text { Physical activity measured } \\
\text { using questionnaires at four } \\
\text { defined time periods: at } \\
\text { menopause, } 5 \text { and } 10 \text { years } \\
\text { after menopause, and at age } \\
72 \text {. } \\
\text { A. Active women (>30 min/ } \\
\text { day) - mean ( } 95 \% \text { CI) number } \\
\text { of hours of physical activity per } \\
\text { week: } \\
\text { Baseline: } 9.0 \text { ( } 7.8 \text { to 10.2) } \\
\text { Average post-menopausal }\end{array}$ & $\begin{array}{l}\text { 1. Forearm BMC }(\mathrm{mg} / \\
\mathrm{cm}) \\
\text { 2. Forearm bone } \\
\text { mineral apparent } \\
\text { density }\left(\mathrm{mg} / \mathrm{cm}^{3}\right)\end{array}$ & $\begin{array}{l}\text { Mean }(95 \% \mathrm{Cl}) \text { average } \\
\text { annual changes } \\
\text { 1. Forearm bone mineral } \\
\text { content }(\mathrm{mg} / \mathrm{cm}) \text { : } \\
\text { A. }-1.2(-1.3 \text { to }-1.1) \\
\text { B. }-1.6(-1.9 \text { to }-1.3) \\
\text { Mean differences } \\
0.4(0.1 \text { to } 0.6)^{*} \\
\text { 2. Forearm bone mineral } \\
\text { apparent density } \\
\text { A. }-1.8(-1.9 \text { to }-1.7) \\
\text { B. }-2.0(-2.2 \text { to }-1.7)\end{array}$ \\
\hline
\end{tabular}


Table 4 Description of included studies investigating the association between physical activity and osteoporosis employing an observational design (Continued)

\begin{tabular}{|c|c|c|c|c|c|}
\hline $\begin{array}{l}\text { Reference } \\
\text { Overall Risk of } \\
\text { Bias }\end{array}$ & $\begin{array}{l}\text { Study design } \\
\text { Included / Analysed }\end{array}$ & $\begin{array}{l}\text { Participants ( } n \text {, } \\
\text { age mean (SD), } \\
\% \text { women, } \\
\text { setting, health } \\
\text { status) }\end{array}$ & Exposure & Outcomes & Results \\
\hline
\end{tabular}

recruited at age 48 and followed up for 25 years Female: 100\%

ableicher 2013 (CHAMP study) Overall risk of bias: Low [80]

Longitudinal cohort study (2 years follow-up) $1,705 / 1,122$
Greendale 1995 Retrospective study (Rancho Bernardo 1,703

study)

Overall risk of

bias: Low [49]

physical activity: 8.7 (7.6 to 9.8)

Physical activity at age 72: 8.2

(6.9 to 9.4)

B. Inactive women $(<30 \mathrm{~min} /$ day) - mean $(95 \%$ Cl) number of hours of physical activity per week:

Baseline: 3.0 (1.6 to 4.4)

Average post-menopausal phys-

ical activity: 2.0 (1.4 to 2.5)

Physical activity at age 72: 1.2

(0.5 to 1.9 )

Classification: total physical activity

Setting:

Community;

Australia

Health status:

Healthy

$\boldsymbol{n}=1,122$

Age: $76.2(5.1)$

range 70-97

Female: $0 \%$
A. Walking for daily exercise was self-reported and measured in kilometres per day

A. Walk daily $>0$ to $\leq 1 \mathrm{~km}$

B. Walk daily $>1$ to $\leq 2 \mathrm{~km}$

C. Walk daily $>2$ to $\leq 4 \mathrm{~km}$

D. Walk daily $>4 \mathrm{~km}$

Classification: planned physical activity (exercise)

B. Physical Activity Scale for the Elderly (units)

Classification: total physical activity
1. Total hip BMD

2. Total hip BMC

(n)

Multiple regression coefficient $(95 \% \mathrm{Cl})$ Reference: no walking Leisure-time walking 1. Total hip BMD A. $0.09(-0.18$ to 0.36$), p=$ 0.5

B. $0.18(-0.06$ to 0.41$), p=$ 0.1

C. 0.29 (0.06 to 0.52$), p=$ $0.01^{*}$

D. $0.19(-0.1$ to 0.49$), p=$ 0.2

2. Hip BMC: NR

Age adjusted annualised percentage change in total hip BMD per unit change

General physical activity

1. Total hip BMD

$-0.01(-0.09$ to 0.07$)$

2. Hip BMC: NR

\section{Setting:}

Community;

United States

Health status:

Healthy

$\boldsymbol{n}=1,703$

Age: 73

Female: $60 \%$
Lifetime leisure physical activity, 1. Total hip BMD calculated based on leisure time physical activity (collected retrospectively via questionnaire) for the past year age 30 years and age 50 years Exercise level: classified by the highest level of exercise performed for at least 15 minutes per session at least three times per week.

Participants were divided into levels of physical activity according to the tertiles

A. Low

B. Medium

C. High

Classification: planned

physical activity (exercise)

BMD

BMD
Adjusted mean ( $p$-value 2. Intertrochanter BMD for comparison A vs $\mathrm{C}$

1. Total hip BMD ( $p=$ $0.002)^{*}$

A. Low: 0.8241

4. Greater trochanter

5. Lumbar spine (L1-4) $\quad$ B. Medium: 0.8367

6. Distal radius BMD 2. Intertrochanter BMD

7. Midshaft radius BMD $(p=0.007)^{*}$

A. Low: 0.9631

B. Medium: 0.9769

C. High: 0.9908

3. Femoral neck BMD ( $p=$ $0.003)^{*}$

A. Low: 0.6597

B. Medium: 06716

C. High: 0.6819

4. Greater trochanter BMD

$(p=0.0001)^{*}$

A. Low: 0.5969

B. Medium: 0.6093

C. High: 0.6248

5. Lumbar spine (L1-4)

BMD

A. Low: 0.9324

B. Medium: 0.9612 
Table 4 Description of included studies investigating the association between physical activity and osteoporosis employing an observational design (Continued)

\begin{tabular}{|c|c|c|c|c|c|}
\hline $\begin{array}{l}\text { Reference } \\
\text { Overall Risk of } \\
\text { Bias }\end{array}$ & $\begin{array}{l}\text { Study design } \\
\text { Included / Analysed }\end{array}$ & $\begin{array}{l}\text { Participants ( } n \text {, } \\
\text { age mean (SD), } \\
\text { \% women, } \\
\text { setting, health } \\
\text { status) }\end{array}$ & Exposure & Outcomes & Results \\
\hline
\end{tabular}

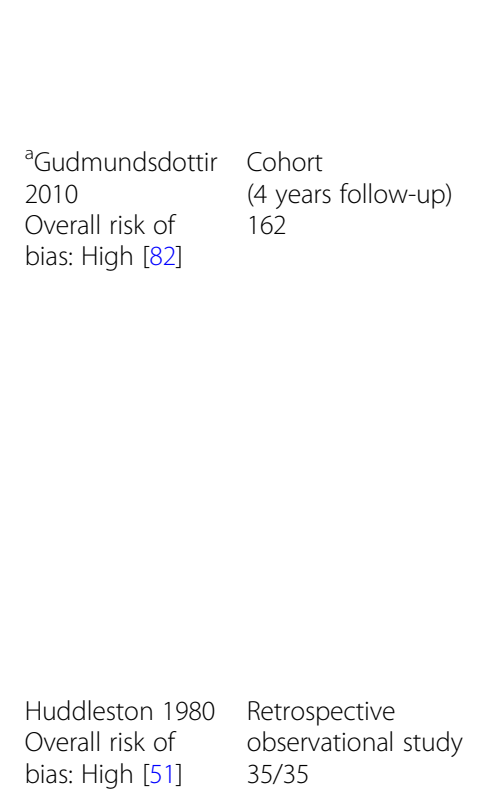

${ }^{a}$ Kemmler (2016)

Bone

(Erlangen Fitness and Osteoporosis Prevention Study) Overall risk of bias: High [83, 93]

Retrospective secondary analysis of the intervention group of a quasi-randomised trial (16 years followup)

\author{
Setting: \\ Community; \\ Iceland \\ Health status: \\ Healthy \\ A. Physical \\ activity \\ performed $\leq 3$ \\ per week \\ $\boldsymbol{n}=41$ (analysed) \\ B. Physical \\ activity \\ performed $>3$ \\ per week \\ $\boldsymbol{n}=111$ (analysed) \\ Age: 75 \\ Female: 100\% \\ Setting: \\ Community; \\ United States \\ Health status \\ Healthy tennis \\ athletes \\ $\boldsymbol{n}=35$ \\ Age: range 70-79 \\ Female: $0 \%$

\section{Lifetime tennis exposure in} \\ athletes with tennis experience \\ ranging from 25 to 72 years \\ Results were presented for: \\ A. Playing arm \\ B. Non-playing arm \\ C. Comparison with data for a \\ "normal male population" \\ Classification: planned \\ physical activity (sport - tennis)
}

Radius midshaft BMC

Femoral neck BMD 2. Total trochanter BMD

3. Total hip BMD

C. High: 0.9479

6. Distal radius BMD: NR

7. Midshaft radius $B M D$ :

NR

Change score; mean \%

change (SE)

1. Femoral neck

A. $-1.3(1.1)$

B. $-0.2(0.9)$

$\beta: N R$

2. Total trochanter BMD

A. $-1.5(0.8)$

B. $-1.2(0.7)$

$\beta=0.22$, non-significant $p$

value

3.Total hip BMD

Mean (SE)

A. -1.4 (0.8)

B. $-1.1(0.7)$

$\beta=0.19$, non-significant $p$ value

1. Radius BMC

$4 \%$ to $33 \%$ greater for the playing arms as compared with the

nonplaying arms

The mean difference

between the playing arm and nonplaying arm: 13\%

Reference data suggest

difference between

dominant and

nondominant

BMC values of $6 \%$ to $9 \%$

Setting:

Community:

Germany

Health status

Osteopenia

A. Exercise

group:

$n=55$

Age: 55.1 (3.4)

Female: 100\%

Rikkonen $2010 \quad$ Cohort (15 years

(Kuopio follow-up)

Osteoporosis Risk 8560

Factor and

Prevention study)

Overall risk of

bias: Low [66]
Exercise group: Supervised group class (aerobic dance exercise, jumping and resistance exercise) + Home training (rope skipping, isometric and dynamic resistance exercise and stretching/ flexibility exercise) five months after study started; 49 to 50 weeks/year throughout the 16 years Exercise frequency (ExFreq) session/week/16 years

Classification: planned physical activity (exercise)

1. Lumbar spine BMD

2. Total hip BMD

1. Femoral neck BMD

2. Trochanter BMD BMD
Linear mixed-effect regression analysis. Marginal effect $(95 \% \mathrm{Cl})$

1. Lumbar spine BMD: 0.035 (0.024 to 0.045$)$

2. Total hip BMD: 0.015 (0.005 to 0.026)

Minimum effective dose of exercise (training sessions/week)

1. Lumbar spine: 2.11

(2.06 to 2.12)

2. Total hip BMD: 2.22

(2.00 to 2.78 )

Beta \pm SE (quartile IV vs inactive)

3. Ward's triangle BMD 1. Femoral neck BMD

4. Lumbar spine (L2-4) $1.752 \pm 0.493$

2. Trochanter BMD

$1.783+0.581$

3. Ward's triangle BMD
Setting:
Community;
Finland
Health status:
Healthy
$\boldsymbol{n}=8560$
(analysed)
A. Physical activity quartile
Age: 52.1 (2.9)
B. Physical
eisure-time physical activity (self-reported) collected at 5 years intervals
A. 15-year average PA, hours/ week: 0.35 (0.35)
B. 15-year average PA, hours/ week: 1.7 (0.39)
C. 15-year average PA, hours/ week: 3.2 (0.54)
D. 15-year average PA, hours/ week: 7.0 (2.9)
Classification: leisure-time

$2.412 \pm 0.723$

4. Lumbar spine (L2-4)

BMD

$0.040 \pm 0.649$

All results were significant 
Table 4 Description of included studies investigating the association between physical activity and osteoporosis employing an observational design (Continued)

\begin{tabular}{|c|c|c|c|c|}
\hline $\begin{array}{l}\text { Reference } \\
\text { Overall Risk of } \\
\text { Bias }\end{array}$ & $\begin{array}{l}\text { Study design } \\
\text { Included / Analysed }\end{array}$ & $\begin{array}{l}\text { Participants (n, } \\
\text { age mean (SD), } \\
\text { \% women, } \\
\text { setting, health } \\
\text { status) }\end{array}$ & Outcomes & Results \\
\hline & & $\begin{array}{l}\text { activity quartile } \\
\text { II } \\
\text { Age: } 52.0(2.9) \\
\text { C. Physical } \\
\text { activity quartile } \\
\text { III } \\
\text { Age: } 52.2(2.9) \\
\text { D. Physical } \\
\text { activity quartile } \\
\text { IV } \\
\text { Age: } 52.3(2.8) \\
\text { Female: } 100 \%\end{array}$ & $\begin{array}{l}\text { physical activity (exercise, trans- } \\
\text { portation, sport) }\end{array}$ & $\begin{array}{l}\text { (except for lumbar spine) } \\
\text { and suggest a positive } \\
\text { effect of physical activity } \\
\text { on BMD. }\end{array}$ \\
\hline
\end{tabular}

$B M C$ bone mineral content ( $\mathrm{g}$ unless specified), $B M D$ bone mineral density $(\mathrm{g} / \mathrm{cm} 2)$. Where studies reported effect estimates with differing degrees of adjustment for confounders in different models, we used the estimate from the most adjusted model

andicate studies that were found in the updated search

*indicates statistically significant between-group difference at $p<0.05$.

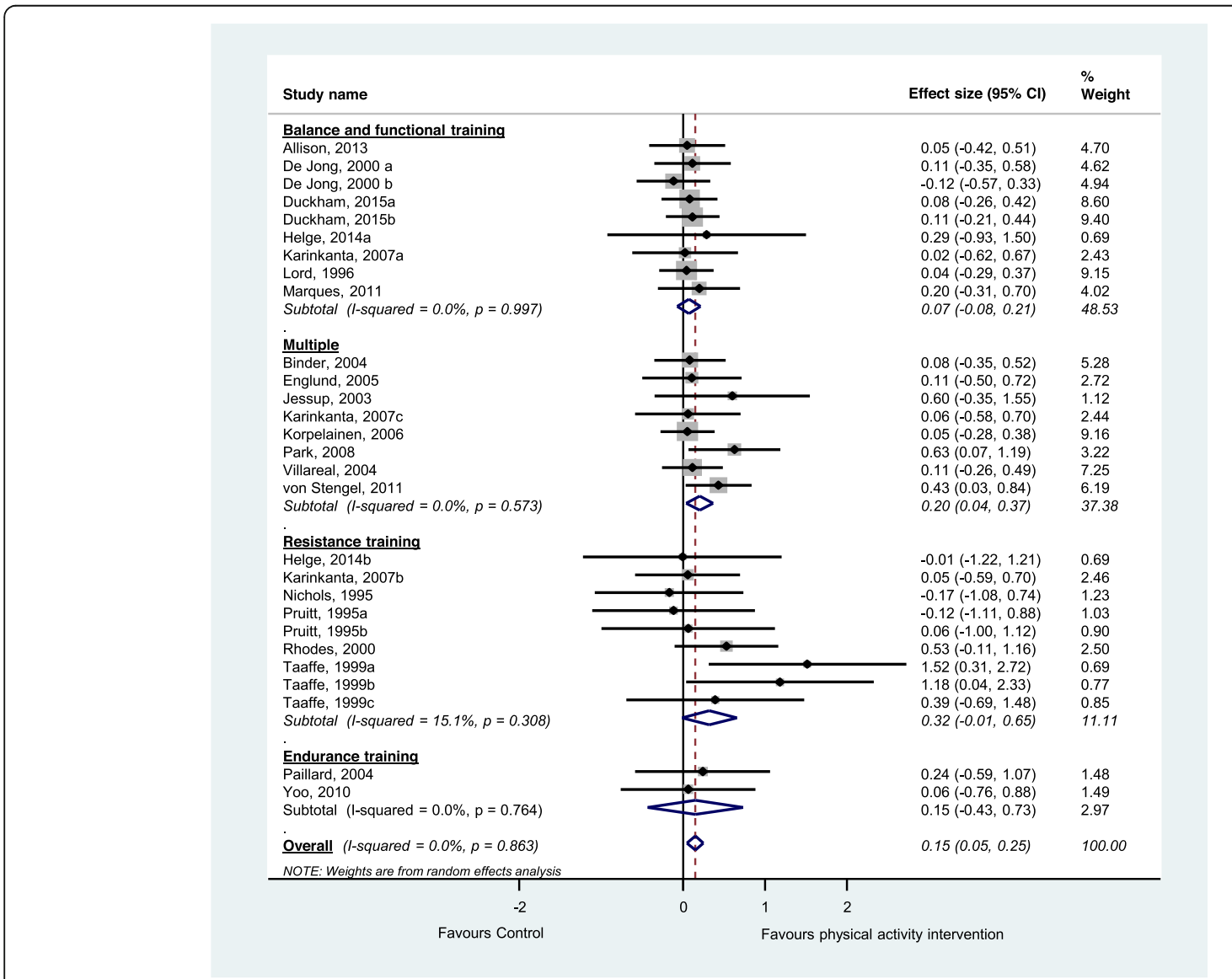

Fig. 2 Effect size (95\% confidence interval) of physical activity interventions on the main outcome by pooling data from 20 studies comparing physical activity versus control using random-effects meta-analysis $(n=1560)$ 
Table 5 Summary of findings and quality of evidence assessment for physical activity interventions vs control

\begin{tabular}{|c|c|c|c|c|c|c|c|}
\hline \multirow[t]{2}{*}{ Outcome } & \multicolumn{2}{|l|}{ Summary of findings } & \multicolumn{5}{|c|}{ Quality of evidence assessment (GRADE) } \\
\hline & Effect size $^{a}(95 \% \mathrm{Cl})$ & n (trials) & Study limitations & Imprecision & Inconsistency & Publication bias & Quality \\
\hline Main outcome & $0.15(0.05$ to 0.25$)$ & $1560(20)$ & $-1^{b}$ & None & None & None & Moderate \\
\hline Femoral neck BMD & $0.09(-0.03$ to 0.21$)$ & $1032(14)$ & $-1^{b}$ & None & None & $-1^{c}$ & Low \\
\hline Lumbar spine BMD & $0.17(0.04$ to 0.30$)$ & $874(11)$ & $-1^{b}$ & None & None & None & Moderate \\
\hline
\end{tabular}

$B M D$ bone mineral density, $n$ number of participants

${ }^{a}$ pooled standardised effect size and $95 \%$ confidence intervals (positive value favours physical activity interventions)

b $>50 \%$ of studies in the meta-analysis had a PEDro score $<6 / 10$

'Serious small study effects suggested by visual inspection of funnel plot or sensitivity analysis investigating the impact of removal of small studies on pooled estimate

\section{Type and domain of physical activity}

Meta-regression was undertaken to investigate whether the inclusion of any particular component in a program was associated with greater overall effects. There was a trend for greater effects of programs that included multiple exercise or resistance types on femoral neck BMD ( $p=0.059$ for the difference in effects) with significant effects for the programs that involved multiple exercise types or resistance exercise (standardised effect $0.24,95 \%$ CI 0.03 to 0.44 ) but not for programs that did not (standardised effect - 0.02, 95\%
CI -0.19 to 0.15 ). Similarly, there was a trend of greater effects in programs that included multiple exercise and resistance types on lumbar spine BMD ( $p=0.256$ for the difference in effects) with significant effects for the programs that involved multiple exercise types or resistance exercise (standardised effect 0.26 , $95 \%$ CI 0.04 to 0.48 ) but not for programs that did not (standardised effect $0.09,95 \% \mathrm{CI}-0.11$ to 0.30). There was no evidence of differential effects by the inclusion of bone loading exercises or balance exercises.

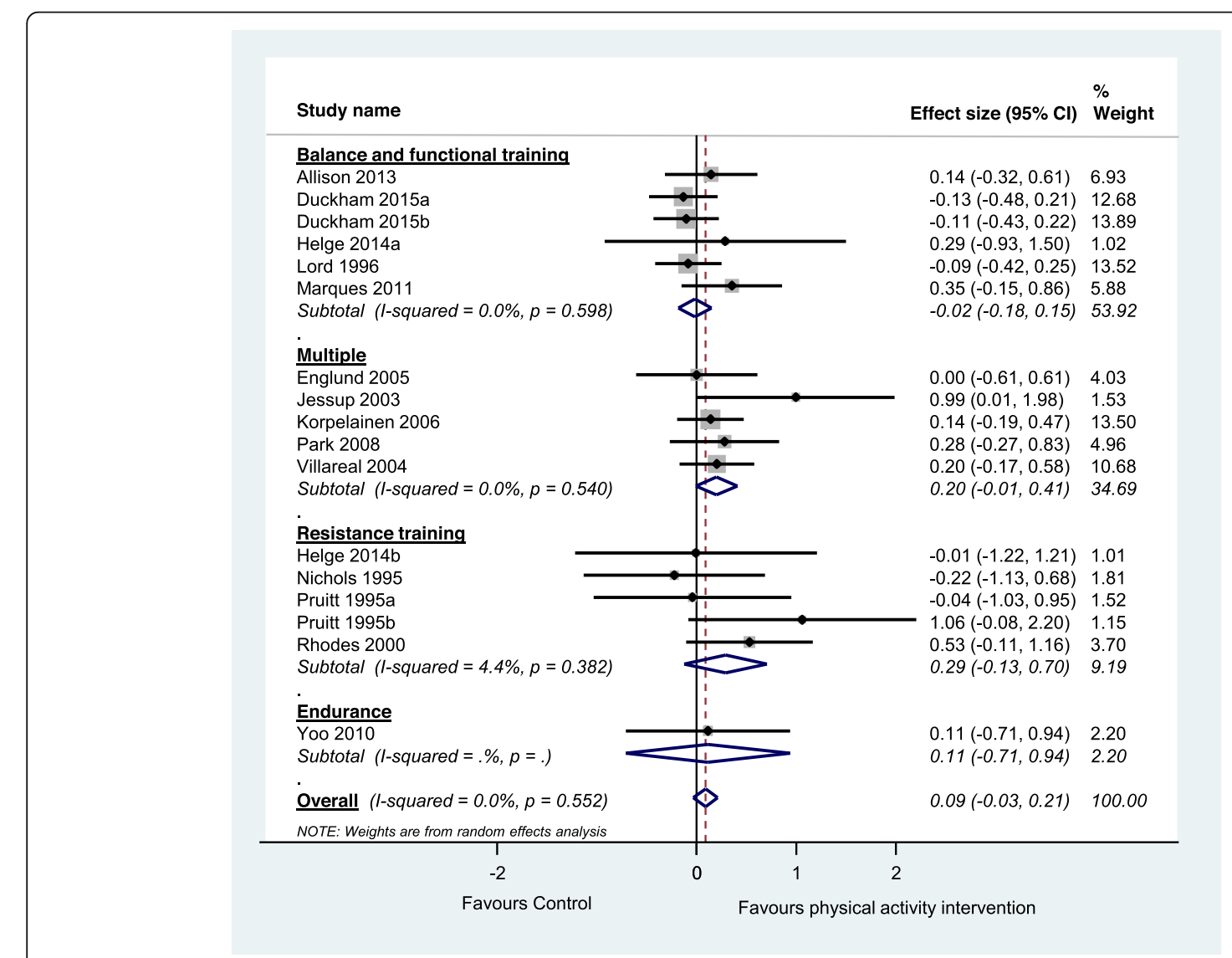

Fig. 3 Effect size (95\% confidence interval) of physical activity interventions on the femoral neck bone mineral density by pooling data from 14 studies comparing physical activity versus control using random-effects meta-analysis $(n=1032)$ 


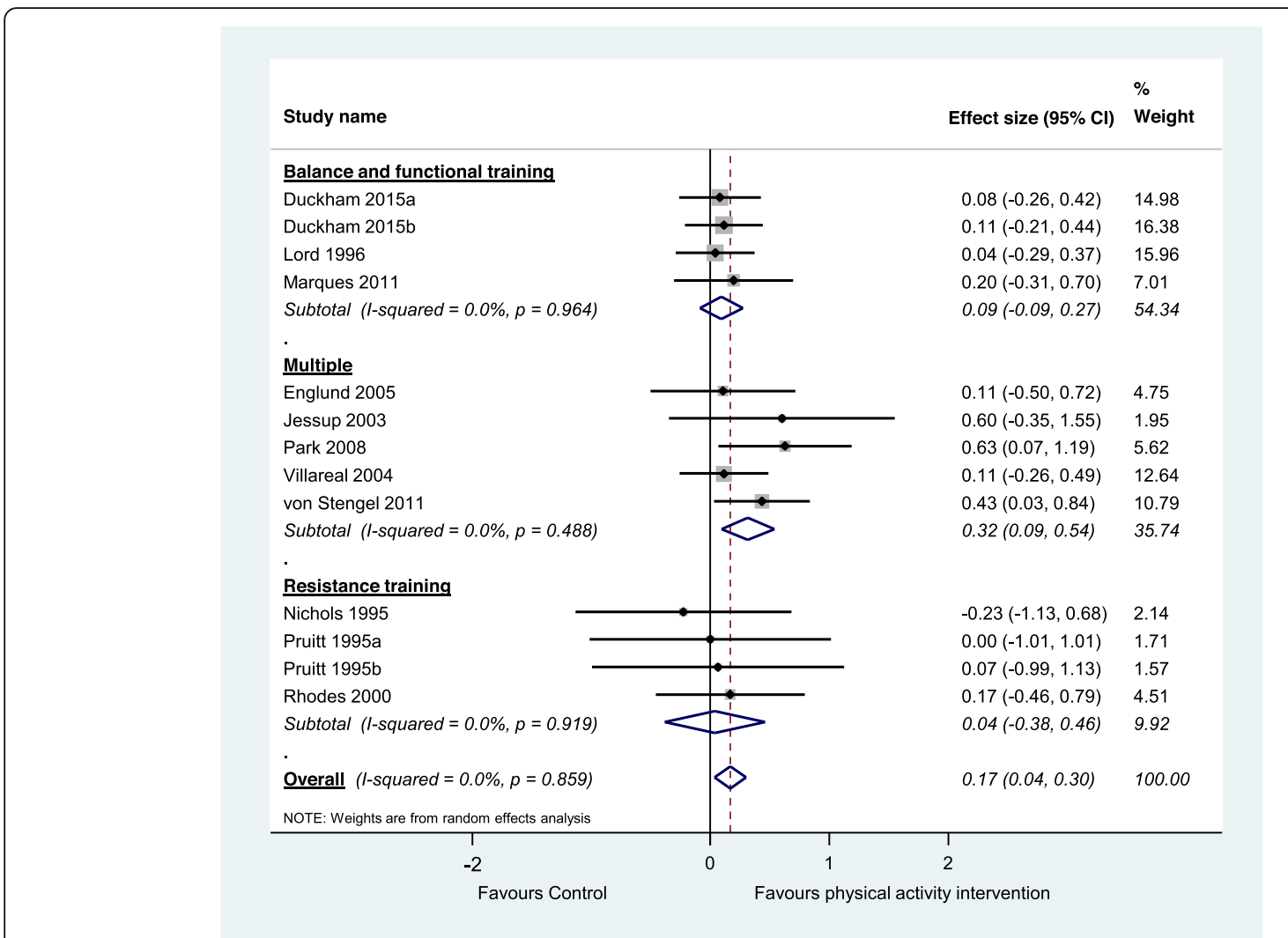

Fig. 4 Effect size (95\% confidence interval) of physical activity interventions on the lumbar spine bone mineral density by pooling data from 11 studies comparing physical activity versus control using random-effects meta-analysis $(n=874)$

Meta-analysis revealed that programs including multiple exercise types had a significant impact on bone when the main outcome from each study was pooled (standardised effect size $0.20,95 \%$ CI 0.04 to $0.37, n=8$ trials; Fig. 2), as well as on a pooled analysis of lumbar spine BMD (standardised effect size 0.32 95\% CI 0.09 to $0.54, n=5$ trials; Fig. 4). Meta-analysis findings did not quite reach significance for programs including multiple exercise types for femoral neck BMD (standardised effect size $0.20,95 \%$ CI -0.01 to $0.41, n=5$ trials, Fig. 3 ).

Meta-analysis revealed that the effects of balance and functional exercises did not reach significance when the main outcome from each study was pooled (Fig. 2), or when femoral neck BMD (Fig. 3) and lumbar spine BMD (Fig. 4) were analysed. Meta-analysis also revealed that the pooled effects of resistance training as a single exercise component was not significant for the overall analysis (Fig. 2), femoral neck BMD (Fig. 3) and lumbar spine BMD outcomes (Fig. 4).

There were 11 studies comparing two or more forms of physical activity interventions (Table 2). The comparisons investigated by the studies included: balance vs resistance [42, 50, 53], balance vs endurance [44], multiple vs resistance [53, 90], 3D vs resistance [69, 75], multiple vs balance [53], endurance vs multiple [67], multiple vs multiple [55] and endurance vs resistance vs multiple [87]. Only one trial found a statistically significant difference when endurance was compared to resistance or to multiple, with results favouring endurance. None of the remaining studies found a statistically significant difference between the groups and there was no clear pattern of superiority of one form of physical activity in relation to the others. Overall the studies investigated a small sample of participants (median $=58$; range 23 to 176 participants analysed) and may have lacked statistical power to detect differences between forms of physical activity interventions.

The programs used in the randomised controlled trials that detected such impacts involved weightbearing exercises that challenged balance and function, plus additional components (such as added resistance and/or endurance training) and were of a relatively high dose $(60+$ mins, $2+$ times per week) and duration (1+ years). For example the study by Bunout [45] involved a $1 \mathrm{~h}$ session of chair stands, squats, step-ups in a stair, arm pull-ups, respiratory muscle training with 15 -min walking periods before and after these exercises, and was undertaken twice a week, with the intensity graded by a specialised coach using the Borg scale and lasted for 72 weeks. The 
study by Jessup [52] also involved multiple components, was undertaken three times a week with 60-90 min per session plus 30-45 min of walking and involved resistance training using a weighted vest.

Taken together, these results suggest that interventions involving a combination of multiple exercise types or resistance exercise may improve bone health and prevent osteoporosis in older people.

\section{Exploration of the impact of study quality}

Exploratory meta-regression did not reveal a differential effect of studies that scored less or more than 6 on the PEDro scale $(p=0.667)$.

\section{Discussion}

\section{Summary of main results}

This review includes 59 studies and of these, 20 randomised controlled trials with 1560 participants contributed to the evidence for the comparison of physical activity interventions with control on the main studies outcome. There is moderate quality evidence that physical activity has a significant but small effect on bone health and particularly in lumbar BMD. The level of evidence is lower for femoral neck BMD, where a small and non-significant effect was found. Programs involving higher doses and multiple exercise types or resistance exercise appear to be more effective. Although it is unclear whether an effect of this magnitude is meaningful for clinicians or patients, overall our results suggest that physical activity probably plays a role in the prevention of osteoporosis.

\section{Interpretation and implications of the findings}

The aim of this review was to investigate the effect of physical activity on osteoporosis prevention in older people. However, none of the studies included in this review reported diagnosis of osteoporosis as an outcome measure. The most commonly reported outcome was $\mathrm{BMD}$, which is commonly used to define osteoporosis. According to the WHO criteria, osteoporosis is defined as a BMD that lies 2.5 standard deviations or more below the average value for young healthy women (a Tscore of $<-2.5$ SD) [100]. Low BMD is one of several risk factors for fractures [101, 102], the main clinical manifestation of osteoporosis. Previous longitudinal studies have indicated the contribution of BMD to fracture, with a one standard deviation decrease in BMD resulting in 2 to 3.5 times greater risk of fracture [103]. A recent individual patient data review including data from 91,779 participants from multiple randomised controlled trials has demonstrated that treatment-related BMD changes are strongly associated with fracture reductions in trials of interventions for osteoporosis, supporting the use of BMD as a surrogate outcome for fracture in randomised controlled trials [104].

Although this review has revealed a small effect of physical activity on bone health, this finding should be interpreted considering the additional benefits of physical activity on other risk factors for fractures in older people, such as falls [105], poor strength [102] and balance [106]. Taken together, these findings suggest that it is likely that physical activity generates clinically meaningful benefits for the prevention of osteoporosis in older people. Clinicians and policy makers should consider these findings when prescribing exercises to older patients without a diagnosis of osteoporosis or making public health decisions.

Although the optimal exercise intervention to prevent osteoporosis has not been defined, our sub-group analysis and meta-regression results suggest that those that included multiple exercises types and resistance exercises had greater effects. These findings are in agreement with a previous review that found that the most effective intervention for spine BMD in postmenopausal women was combination exercise programs (pooled mean difference 3.22; $95 \%$ CI 1.80 to 4.64) [17], however this pooled analysis included participants with and without osteoporosis, unlike our review that focused on prevention only.

Our findings also suggested a dose-response relationship with typical programs that showed an impact being undertaken for $60+\min , 2-3$ times/week for $7+$ months. The studies investigating exercises programs had a median duration of 12 months and it is likely that longer exercise programs would have greater effects on bone health, as suggested by the longitudinal studies. Although the confidence in these findings is not high, these results are in line with guideline recommendations that participants without osteoporosis should engage regularly in physical activity (at least 2-3 times/week) and programs should include a combination of exercises types [107].

Although previous reviews have suggested that bone loading (high impact) exercises and non-weight-bearing high force exercise alone provide benefits to bone health $[17,18,107]$, we were not able to confirm these results in this review. Since previous reviews have used different classification systems for physical activity interventions, direct comparisons are not possible. Additionally, in the present review none of the included studies investigated bone loading alone. Other factors that might explain differences in our findings in relation to previous reviews include the fact that previous reviews have investigated younger participants, have pooled together studies investigating the effect of physical activity on prevention (i.e. in participants without osteoporosis) and management (i.e. in participants with osteoporosis at baseline) of osteoporosis. 


\section{Strengths and potential biases in the review process}

This review provides a comprehensive overview of the evidence on the role of physical activity on osteoporosis prevention in older people, without limits by gender, body parts, or physical activity type. Additionally, we were able to perform analysis according to physical activity types and to explore the effect of dose on the physical activity effects.

The initial aim of this review was to summarise the evidence of physical activity on prevention of osteoporosis in older people by conducting a review of systematic reviews. However, since no reviews were found we included the relevant studies identified from the reviews. We decided to expand the search for individual studies, since the initial search was targeted at reviews, and it was possible that we had missed important studies, particularly recently-published ones (the most recent included study in the report was published in 2015). We were able to include 19 additional studies with our expanded search. We also updated our search for reviews in PubMed and conducted searches in three additional databases. We found 4 additional studies and although our main results remained unchanged with the addition of these studies, our search was focused on reviews, rather than individual studies, and it is possible that we might have missed relevant studies that were not included in the identified reviews.

We only included studies investigating the effects of physical activity for the prevention of osteoporosis and therefore excluded studies where all participants had been diagnosed with osteoporosis. Most studies did not use the absence of osteoporosis at baseline as an inclusion criterion. Therefore, it is likely that the studies investigated samples of people with mixed bone health status. One review author classified the exercise interventions using the ProFaNE guidelines [30] and a second one checked the classification. We recognise there is some subjectivity in this classification system, particularly for those interventions containing more than one category of exercise.

\section{Unanswered questions and future research}

This review has focused on older people only but it is likely that exposure to physical activity earlier in life plays a key role in bone deposition and thereby, osteoporosis prevention, as indicated by previous studies [108], however this was beyond the scope of this review. We focused on prevention of osteoporosis, and therefore excluded studies where all participants were diagnosed with osteoporosis. Since bone health is a continuum, the inclusion of studies of people with existing osteoporosis would provide additional understanding of the effect of physical activity on osteoporosis but was also beyond the scope of this review. The investigation of the effects of physical activity on fragility fractures was not covered in this review. However, since fragility fracture is the main clinical manifestation of osteoporosis [1], future research should focus on investigating the impact of physical activity on this outcome. Lastly, previous reviews investigating the effects of physical activity programs on osteoporosis have used different classification systems for physical activity. Future studies should focus on using standardised classification systems to facilitate comparison of results across reviews.

The overall quality of included studies varied and overall was low (median PEDro score $=5$ ), and this has been taken into account in the GRADE approach, where all three analysis were downgraded on the basis of study limitations. Although meta-regression did not reveal a differential effect when studies were stratified as high or low quality, future studies should improve the methodological quality of studies, particularly in terms of followup rate, allocation concealment and intention to treat analysis, which were the main limitations of studies in this review. Additionally, the trials had a small sample size (median $=50)$ and relatively short follow-up (median follow-up length $=12$ months). Future studies should investigate larger samples and have longer follow-up duration.

\section{Conclusions}

In summary, while the results need to be treated with some caution, the studies included in this review suggest that physical activity is likely to play a role in the prevention of osteoporosis in older people. The level of evidence is higher for lumbar spine BMD (than for femoral neck BMD) and higher dose programs and those involving multiple exercises types or resistance exercise appear to be more effective.

\section{Supplementary Information}

Supplementary information accompanies this paper at https://doi.org/10. 1186/s12966-020-01040-4.

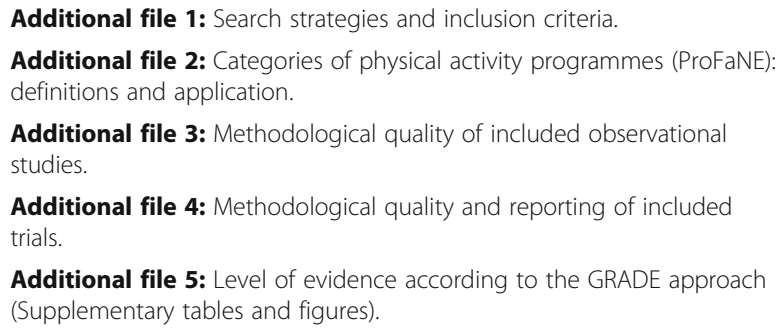

Additional file 3: Methodological quality of included observational studies.

Additional file 4: Methodological quality and reporting of included trials.

Additional file 5: Level of evidence according to the GRADE approach (Supplementary tables and figures).

\section{Abbreviations}

BMC: Bone mineral content; BMD: Bone mineral density; Cl: Confidence interval; GDG: Guideline Development Group; GRADE: Grading of Recommendations Assessment, Development and Evaluation; PICO: Population, intervention, comparison, outcome; ProFaNE: Prevention of Falls Network Europe; WHO: World Health Organization 


\section{Acknowledgements}

We are grateful to A/Prof Maureen Ashe and co-authors for providing a list of trials included in their upcoming Cochrane review on bone health in men.

\section{Authors' contributions}

MBP led the review and searching process, conducted the screening, data extraction and led the writing of the article. JO reviewed articles in the case of disagreements, conducted data extraction and performed the statistical analysis. NF contributed to risk of bias assessment, GRADE and interpretation of results. WK conducted the screening, data extraction and contributed to risk of bias assessment. CS and AB provided advice and guidance in all aspects of the review. All authors contributed to the review plan, searching process, article draft and approved the final version for submission.

\section{Funding}

This review was prepared for and funded by the World Health Organization, to contribute to the development of the Guidelines on physical activity and sedentary behaviour (2020). It was submitted to the Guideline Development Group and considered as they formulated their recommendations. MBP and CS receive salary funding from the Australian National Health and Medical Research Council fellowships.

The WHO guideline development group decided on the scope of the review, the PICO (Population, Intervention, Comparison, Outcome) question, and the search strategy. WHO had no role in the conduct of this review, data analysis, interpretation of the re-sults, manuscript preparation, or decision to publish.

\section{Availability of data and materials}

All data generated or analysed during this study are included in this published article and its supplementary information files.

\section{Ethics approval and consent to participate}

Not applicable. This study is a systematic review and no ethics approval was required to conduct this review.

\section{Consent for publication}

Not applicable.

\section{Competing interests}

The authors declare that they have no competing interests.

\section{Author details}

'Institute for Musculoskeletal Health, The University of Sydney and Sydney Local Health District, Sydney, Australia. ${ }^{2}$ School of Public Health, Faculty of Medicine and Health, The University of Sydney, Sydney, Australia. ${ }^{3}$ Prevention Research Collaboration, Sydney School of Public Health, The University of Sydney, Sydney, Australia.

Received: 27 May 2020 Accepted: 20 October 2020 Published online: 26 November 2020

\section{References}

1. The Royal Australian College of General Practitioners and Osteoporosis Australia. Osteoporosis prevention, diagnosis and management in postmenopausal women and men over 50 years of age. 2 nd ed. East Melbourne: RACGP; 2017.

2. Kanis JA. Assessment of fracture risk and its application to screening for postmenopausal osteoporosis: synopsis of a WHO report. WHO Study Group Osteoporos Int. 1994:4:368-81.

3. Parker D. An audit of osteoporotic patients in an Australian general practice. Aust Fam Physician. 2013;42:423-7.

4. Papaioannou A, Kennedy CC, loannidis G, Sawka A, Hopman WM, Pickard L, et al. The impact of incident fractures on health-related quality of life: 5 years of data from the Canadian multicentre osteoporosis study. Osteoporos Int. 2009;20:703-14.

5. loannidis G, Papaioannou A, Hopman WM, Akhtar-Danesh N, Anastassiades T, Pickard $L$, et al. Relation between fractures and mortality: results from the Canadian multicentre osteoporosis study. Can Med Assoc J. 2009;181:265-71.
6. Watts JJ, Abimanyi-Ochom J, Sanders K. In: Australia O, editor. Osteoporosis costing all Australians: a new burden of disease analysis - 2012 to 2022. Glebe: NSW; 2013.

7. Abimanyi-Ochom J, Watts JJ, Borgstrom F, Nicholson GC, Shore-Lorenti C, Stuart AL, et al. Changes in quality of life associated with fragility fractures: Australian arm of the international cost and utility related to osteoporotic fractures study (AusICUROS). Osteoporos Int. 2015;26:1781-90.

8. National Institute for Health and Care Excellence. 2018 NICE impact report on falls and fragility fractures 2018.

9. Lewiecki EM, Ortendahl JD, Vanderpuye-Orgle J, Grauer A, Arellano J, Lemay $J$, et al. Healthcare policy changes in osteoporosis can improve outcomes and reduce costs in the United States. JBMR Plus. 2019;3:e10192.

10. Caspersen CJ, Powell KE, Christenson GM. Physical activity, exercise, and physical fitness: definitions and distinctions for health-related research. Public Health Rep. 1985;100:126-31.

11. Das P, Horton R. Physical activity-time to take it seriously and regularly. Lancet. 2016:388:1254-5.

12. World Health Organization. Global Strategy on Diet, Physical Activity and Health 2010.

13. National Osteoporosis Society. Strong, Steady and Straight - An Expert Consensus Statement on Physical Activity and Exercise for Osteoporosis. 2018

14. Marin-Cascales E, Alcaraz PE, Ramos-Campo DJ, Rubio-Arias JA. Effects of multicomponent training on lean and bone mass in postmenopausal and older women: a systematic review. Menopause. 2018;25:346-56.

15. Kemmler W, Shojaa M, Kohl M, von Stengel S. Exercise effects on bone mineral density in older men: a systematic review with special emphasis on study interventions. Osteoporos Int. 2018;29:1493-504.

16. de Kam D, Smulders E, Weerdesteyn V, Smits-Engelsman BC. Exercise interventions to reduce fall-related fractures and their risk factors in individuals with low bone density: a systematic review of randomized controlled trials. Osteoporos Int. 2009;20:2111-25.

17. Howe TE, Shea B, Dawson L, Downie F, Murray A, Ross C, et al. Exercise for preventing and treating osteoporosis in postmenopausal women. Cochrane Database Syst Rev. 2011;7:CD000333.

18. Martyn-St James M, Carroll S. A meta-analysis of impact exercise on postmenopausal bone loss: the case for mixed loading exercise programmes. Br J Sports Med. 2009:43:898-908.

19. $\mathrm{Ma} \mathrm{D}, \mathrm{Wu} L, \mathrm{He} Z$. Effects of walking on the preservation of bone mineral density in perimenopausal and postmenopausal women: a systematic review and meta-analysis. Menopause. 2013;20:1216-26.

20. Martyn-St James M, Carroll S. Meta-analysis of walking for preservation of bone mineral density in postmenopausal women. Bone. 2008;43:521-31.

21. Abrahin O, Rodrigues RP, Marcal AC, Alves EA, Figueiredo RC, Sousa EC. Swimming and cycling do not cause positive effects on bone mineral density: a systematic review. Rev Bras Reumatol. 2016;56(4):345-51.

22. Babatunde OO, Bourton AL, Hind K, Paskins Z, Forsyth JJ. Exercise interventions for preventing and treating low bone mass in the forearm: a systematic review and meta-analysis. Arch Phys Med Rehabil. 2020;101(3): 487-511.

23. Bolam KA, van Uffelen JG, Taaffe DR. The effect of physical exercise on bone density in middle-aged and older men: a systematic review. Osteoporos Int. 2013:24:2749-62

24. Manferdelli G, La Torre A, Codella R. Outdoor physical activity bears multiple benefits to health and society. J Sports Med Phys Fitness. 2019:59:868-79.

25. Bennie JA, Pedisic Z, van Uffelen JG, Gale J, Banting LK, Vergeer I, et al. The descriptive epidemiology of total physical activity, muscle-strengthening exercises and sedentary behaviour among Australian adults--results from the National Nutrition and physical activity survey. BMC Public Health. 2016;16:73.

26. World Health Organization. Guidelines for physical activity and sedentary behaviour. Geneva: World Health Organization; 2020.

27. Bull FC, Al-Ansari SS, Biddle S, Borodulin K, Buman MP, Cardon G, et al. World Health Organization 2020 guidelines on physical activity and sedentary behaviour. Br J Sports Med. 2020.

28. Moher D, Liberati A, Tetzlaff J, Altman DG, The PG. Preferred reporting items for systematic reviews and meta-analyses: The PRISMA statement. PLoS Med. 2009;6:e1000097.

29. Liberati A, Altman DG, Tetzlaff J, Mulrow C, Gøtzsche PC, loannidis JPA, et al. The PRISMA statement for reporting systematic reviews and meta-analyses of studies that evaluate healthcare interventions: explanation and elaboration. BMJ. 2009;339:b2700. 
30. Lamb SE, Becker C, Gillespie LD, Smith JL, Finnegan S, Potter R, et al. Reporting of complex interventions in clinical trials: development of a taxonomy to classify and describe fall-prevention interventions. Trials. 2011;12:125.

31. Macedo LG, Elkins MR, Maher CG, Moseley AM, Herbert RD, Sherrington C. There was evidence of convergent and construct validity of physiotherapy evidence database quality scale for physiotherapy trials. J Clin Epidemiol. 2010;63:920-5

32. Maher CG, Sherrington C, Herbert RD, Moseley AM, Elkins M. Reliability of the PEDro scale for rating quality of randomized controlled trials. Phys Ther. 2003:83:713-21.

33. Hayden JA, van der Windt DA, Cartwright JL, Côté P, Bombardier C. Assessing Bias in studies of prognostic factors. Ann Intern Med. 2013;158: 280-6.

34. Guyatt GH, Oxman AD, Vist GE, Kunz R, Falck-Ytter Y, Alonso-Coello P, et al, GRADE: an emerging consensus on rating quality of evidence and strength of recommendations. BMJ. 2008:336:924-6.

35. Guyatt GH, Oxman AD, Kunz R, Woodcock J, Brozek J, Helfand M, et al. GRADE guidelines: 8. Rating the quality of evidence--indirectness. J Clin Epidemiol. 2011;64:1303-10.

36. Guyatt GH, Oxman AD, Vist G, Kunz R, Brozek J, Alonso-Coello P, et al. GRADE guidelines: 4. Rating the quality of evidence--study limitations (risk of bias). J Clin Epidemiol. 2011;64:407-15.

37. Guyatt GH, Oxman AD, Kunz R, Brozek J, Alonso-Coello P, Rind D, et al. GRADE guidelines 6. Rating the quality of evidence--imprecision. J Clin Epidemiol. 2011;64:1283-93.

38. Guyatt GH, Oxman AD, Kunz R, Woodcock J, Brozek J, Helfand M, et al. GRADE guidelines: 7. Rating the quality of evidence--inconsistency. J Clin Epidemiol. 2011;64:1294-302

39. Guyatt GH, Oxman AD, Montori V, Vist G, Kunz R, Brozek J, et al. GRADE guidelines: 5. Rating the quality of evidence--publication bias. J Clin Epidemiol. 2011;64:1277-82.

40. Cumpston M, Li T, Page MJ, Chandler J, Welch VA, Higgins JP, et al. Updated guidance for trusted systematic reviews: a new edition of the Cochrane handbook for systematic reviews of interventions. Cochrane Database Syst Rev. 2019;10:ED000142.

41. Allison SJ, Folland JP, Rennie WJ, Summers GD, Brooke-Wavell K. High impact exercise increased femoral neck bone mineral density in older men: a randomised unilateral intervention. Bone. 2013;53:321-8.

42. Ashe MC, Gorman E, Khan KM, Brasher PM, Cooper DML, McKay HA, et al. Does frequency of resistance training affect tibial cortical bone density in older women? A randomized controlled trial. Osteoporos Int. 2013;24:623-32.

43. Binder EF, Brown M, Sinacore DR, Steger-May K, Yarasheski KE, Schechtman KB. Effects of extended outpatient rehabilitation after hip fracture: a randomized controlled trial. JAMA. 2004;292:837-46.

44. Blumenthal JA, Emery CF, Madden DJ, Schniebolk S, Riddle MW, Cobb FR, et al. Effects of exercise training on bone density in older men and women. J Am Geriatr Soc. 1991:39:1065-70.

45. Bunout D, Barrera G, de la Maza P, Avendano M, Gattas V, Petermann M, et al. The impact of nutritional supplementation and resistance training on the health functioning of free-living Chilean elders: results of 18 months of follow-up. J Nutr. 2001;131:2441S-6S.

46. de Jong N, Chin APMJ, de Groot LC, Hiddink GJ, van Staveren WA. Dietary supplements and physical exercise affecting bone and body composition in frail elderly persons. Am J Public Health. 2000;90:947-54.

47. Duckham RL, Masud T, Taylor R, Kendrick D, Carpenter H, lliffe S, et al. Randomised controlled trial of the effectiveness of community group and home-based falls prevention exercise programmes on bone health in older people: the ProAct65+ bone study. Age Ageing. 2015;44:573-9.

48. Englund $U$, Littbrand $H$, Sondell A, Pettersson U, Bucht G. A 1-year combined weight-bearing training program is beneficial for bone mineral density and neuromuscular function in older women. Osteoporos Int. 2005; 16:1117-23.

49. Greendale G, Barrettconnor E, Edelstein S, Ingles S, Haile R. Lifetime leisure exercise and osteoporosis - The Rancho-Bernardo Study. Am J Epidemiol. 1995;141:951-9.

50. Helge EW, Andersen TR, Schmidt JF, Jorgensen NR, Hornstrup T, Krustrup P, et al. Recreational football improves bone mineral density and bone turnover marker profile in elderly men. Scand J Med Sci Sports. 2014; 24(Suppl 1):98-104.

51. Huddleston AL, Rockwell D, Kulund DN, Harrison RB. Bone mass in lifetime tennis athletes. JAMA. 1980;244:1107-9.
52. Jessup JV, Horne C, Vishen RK, Wheeler D. Effects of exercise on bone density, balance, and self-efficacy in older women. Biol Res Nurs. 2003;4: 171-80.

53. Karinkanta S, Heinonen A, Sievanen H, Uusi-Rasi K, Pasanen M, Ojala K, et al. A multi-component exercise regimen to prevent functional decline and bone fragility in home-dwelling elderly women: randomized, controlled trial. Osteoporos Int. 2007;18:453-62.

54. Kemmler W, von Stengel S, Engelke K, Haberle L, Kalender WA. Exercise effects on bone mineral density, falls, coronary risk factors, and health care costs in older women: the randomized controlled senior fitness and prevention (SEFIP) study. Arch Intern Med. 2010;170:179-85.

55. Kohrt WM, Ehsani AA, Birge SJ Jr. Effects of exercise involving predominantly either joint-reaction or ground-reaction forces on bone mineral density in older women. J Bone Miner Res. 1997;12:1253-61.

56. Kwon Y, Park S, Kim E, Park J. The effects of multi-component exercise training on (Vover-dot-O(2)max, muscle mass, whole bone mineral density and fall risk in community-dwelling elderly women. J Phys Fitness Sports. 2008;57:339-48

57. Lau EM, Woo J, Leung PC, Swaminathan R, Leung D. The effects of calcium supplementation and exercise on bone density in elderly Chinese women. Osteoporos Int. 1992;2:168-73.

58. Lord SR, Ward JA, Williams P, Zivanovic E. The effects of a community exercise program on fracture risk factors in older women. Osteoporos Int. 1996;6:361-7.

59. Marques EA, Mota J, Machado L, Sousa F, Coelho M, Moreira P, et al. Multicomponent training program with weight-bearing exercises elicits favorable bone density, muscle strength, and balance adaptations in older women. Calcif Tissue Int. 2011;88:117-29.

60. McCartney N, Hicks AL, Martin J, Webber CE. Long-term resistance training in the elderly: effects on dynamic strength, exercise capacity, muscle, and bone. J Gerontol A Biol Sci Med Sci. 1995;50:B97-104.

61. McMurdo MET, Mole PA, Paterson CR. Controlled trial of weight bearing exercise in older women in relation to bone density and falls. Br Med J. 1997;314:569.

62. Paillard T, Lafont C, Costes-Salon MC, Riviere D, Dupui P. Effects of brisk walking on static and dynamic balance, locomotion, body composition, and aerobic capacity in ageing healthy active men. Int J Sports Med. 2004;25:539-46.

63. Park H, Kim KJ, Komatsu T, Park SK, Mutoh Y. Effect of combined exercise training on bone, body balance, and gait ability: a randomized controlled study in community-dwelling elderly women. J Bone Miner Metab. 2008;26: 254-9.

64. Pruitt LA, Taaffe DR, Marcus R. Effects of a one-year high-intensity versus low-intensity resistance training program on bone mineral density in older women. J Bone Miner Res. 1995;10:1788-95.

65. Rhodes EC, Martin AD, Taunton JE, Donnelly M, Warren J, Elliot J. Effects of one year of resistance training on the relation between muscular strength and bone density in elderly women. Br J Sports Med. 2000;34:18-22.

66. Rikkonen T, Salovaara K, Sirola J, Karkkainen M, Tuppurainen M, Jurvelin J, et al. Physical activity slows femoral bone loss but promotes wrist fractures in postmenopausal women: a 15-year follow-up of the OSTPRE study. J Bone Miner Res. 2010;25:2332-40.

67. Rikli RE, McManis BG. Effects of exercise on bone mineral content in postmenopausal women. Res Q Exerc Sport. 1990;61:243-9.

68. Sakai A, Oshige T, Zenke Y, Yamanaka Y, Nagaishi H, Nakamura T. Unipedal standing exercise and hip bone mineral density in postmenopausal women: a randomized controlled trial. J Bone Miner Metab. 2010;28:42-8.

69. Shen CL, Williams JS, Chyu MC, Paige RL, Stephens AL, Chauncey KB, et al. Comparison of the effects of tai chi and resistance training on bone metabolism in the elderly: a feasibility study. Am J Chin Med. 2007;35:369-81.

70. Smith EL Jr, Reddan W, Smith PE. Physical activity and calcium modalities for bone mineral increase in aged women. Med Sci Sports Exerc. 1981;13: $60-4$.

71. von Stengel S, Kemmler W, Engelke K, Kalender W. Effects of whole body vibration on bone mineral density and falls: results of the randomized controlled ELVIS study with postmenopausal women. Osteoporos Int. 2011; 22:317-25.

72. Taaffe DR, Duret C, Wheeler S, Marcus R. Once-weekly resistance exercise improves muscle strength and neuromuscular performance in older adults. J Am Geriatr Soc. 1999;47:1208-14.

73. Villareal DT, Binder EF, Yarasheski KE, Williams DB, Brown M, Sinacore DR, et al. Effects of exercise training added to ongoing hormone replacement 
therapy on bone mineral density in frail elderly women. J Am Geriatr Soc. 2003;51:985-90.

74. Villareal DT, Steger-May K, Schechtman KB, Yarasheski KE, Brown M, Sinacore DR, et al. Effects of exercise training on bone mineral density in frail older women and men: a randomised controlled trial. Age Ageing. 2004;33:309-12.

75. Woo J, Hong A, Lau E, Lynn H. A randomised controlled trial of tai chi and resistance exercise on bone health, muscle strength and balance in community-living elderly people. Age Ageing. 2007;36:262-8.

76. Yoo EJ, Jun TW, Hawkins SA. The effects of a walking exercise program on fall-related fitness, bone metabolism, and fall-related psychological factors in elderly women. Res Sports Med. 2010;18:236-50.

77. Korpelainen R, Keinanen-Kiukaanniemi S, Heikkinen J, Vaananen K, Korpelainen J. Effect of impact exercise on bone mineral density in elderly women with low BMD: a population-based randomized controlled 30month intervention. Osteoporos Int. 2006;17:109-18.

78. Bleicher K, Cumming RG, Naganathan V, Seibel MJ, Blyth FM, Le Couteur $D G$, et al. Predictors of the rate of BMD loss in older men: findings from the CHAMP study. Osteoporos Int. 2013;24:1951-63.

79. Foley S, Quinn S, Jones G. Pedometer determined ambulatory activity and bone mass: a population-based longitudinal study in older adults. Osteoporos Int. 2010;21:1809-16.

80. Gudmundsdottir SL, Oskarsdottir D, Indridason OS, Franzson L, Sigurdsson G. Risk factors for bone loss in the hip of 75-year-old women: a 4-year follow-up study. Maturitas. 2010;67:256-61.

81. Kemmler W, von Stengel S, Kohl M. Exercise frequency and bone mineral density development in exercising postmenopausal osteopenic women. Is there a critical dose of exercise for affecting bone? Results of the Erlangen fitness and osteoporosis prevention study. Bone. 2016;89:1-6.

82. Muir JM, Ye C, Bhandari M, Adachi JD, Thabane L. The effect of regular physical activity on bone mineral density in post-menopausal women aged 75 and over: a retrospective analysis from the Canadian multicentre osteoporosis study. BMC Musculoskelet Disord. 2013;14:253.

83. Nakamura K, Oyama M, Saito T, Oshiki R, Kobayashi R, Nishiwaki T, et al. Nutritional and biochemical parameters associated with 6-year change in bone mineral density in community-dwelling Japanese women aged 69 years and older: The Muramatsu study. Nutrition. 2012;28:357-61.

84. Rodriguez-Gomez I, Manas A, Losa-Reyna J, Rodriguez-Manas L, Chastin SFM, Alegre LM, et al. Compositional influence of movement behaviors on bone health during aging. Med Sci Sports Exerc. 2019:51:1736-44.

85. Shephard RJ, Park H, Park S, Aoyagi Y. Objective longitudinal measures of physical activity and bone health in older Japanese: the Nakanojo study. J Am Geriatr Soc. 2017;65:800-7.

86. Svejme O, Ahlborg HG, Karlsson MK. Physical activity reduces bone loss in the distal forearm in post-menopausal women--a 25-year prospective study. Scand J Med Sci Sports. 2014;24:159-65.

87. Armamento-Villareal R, Aguirre L, Waters DL, Napoli N, Qualls C, Villareal DT. Effect of aerobic or resistance exercise, or both, on bone mineral density and bone metabolism in obese older adults while dieting: a randomized controlled trial. J Bone Miner Res. 2020;35:430-9.

88. Armamento-Villareal R, Sadler C, Napoli N, Shah K, Chode S, Sinacore DR, et al. Weight loss in obese older adults increases serum sclerostin and impairs hip geometry but both are prevented by exercise training. J Bone Miner Res. 2012;27:1215-21.

89. Bemben DA, Bemben MG. Dose-response effect of 40 weeks of resistance training on bone mineral density in older adults. Osteoporos Int. 2011;22: 179-86.

90. Chan DC, Chang CB, Han DS, Hong CH, Hwang JS, Tsai KS, et al. Effects of exercise improves muscle strength and fat mass in patients with high fracture risk: a randomized control trial. J Formos Med Assoc. 2018;117:572-82.

91. Kemmler W, Engelke K, von Stengel S. Long-term exercise and bone mineral density changes in postmenopausal women-are there periods of reduced effectiveness? J Bone Miner Res. 2016;31:215-22.

92. Kemmler W, von Stengel S, Bebenek M, Engelke K, Hentschke C, Kalender WA. Exercise and fractures in postmenopausal women: 12-year results of the Erlangen fitness and osteoporosis prevention study (EFOPS). Osteoporos Int. 2012;23:1267-76

93. Kim SH, Seong DH, Yoon SM, Choi YD, Choi E, Song Y, et al. The effect on bone outcomes of home-based exercise intervention for prostate Cancer survivors receiving androgen deprivation therapy: a pilot randomized controlled trial. Cancer Nurs. 2018;41:379-88.
94. Korpelainen R, Keinanen-Kiukaanniemi S, Nieminen P, Heikkinen J, Vaananen $\mathrm{K}$, Korpelainen J. Long-term outcomes of exercise: follow-up of a randomized trial in older women with osteopenia. Arch Intern Med. 2010; 170:1548-56.

95. Winters-Stone KM, Dobek JC, Bennett JA, Maddalozzo GF, Ryan CW, Beer TM. Skeletal response to resistance and impact training in prostate cancer survivors. Med Sci Sports Exerc. 2014;46:1482-8.

96. Karinkanta S, Heinonen A, Sievänen H, Uusi-Rasi K, Fogelholm M, Kannus P. Maintenance of exercise-induced benefits in physical functioning and bone among elderly women. Osteoporos Int. 2009;20:665-74.

97. Nichols J, Nelson K, Peterson K, Sartoris D. Bone mineral density responses to high-intensity strength training in active older women. J Aging Phys Act. 1995;3:26-38

98. Snow C, Shaw J, Winters K, Witzke K. Long-term Exercise Using Weighted Vests Prevents Hip Bone Loss in Postmenopausal Women. J Gerontol A Biol Sci Med Sci. 2000;55(9):M489-91.

99. Taaffe DR, Pruitt L, Pyka G, Guido D, Marcus R. Comparative effects of highand low-intensity resistance training on thigh muscle strength, fiber area, and tissue composition in elderly women. Clin Physiol. 1996;16:381-92.

100. World Health O. Assessment of fracture risk and its application to screening for postmenopausal osteoporosis: report of a WHO study group [meeting held in Rome from 22 to 25 June 1992]. Geneva: World Health Organization 1994. https://apps.who.int/iris/handle/10665/39142.

101. Cosman F, de Beur SJ, LeBoff MS, Lewiecki EM, Tanner B, Randall S, et al. Clinician's guide to prevention and treatment of osteoporosis. Osteoporos nt. 2014:25:2359-81.

102. Liu CJ, Latham NK. Progressive resistance strength training for improving physical function in older adults. Cochrane Database Syst Rev. 2009: CD002759.

103. Cummings SR, Black DM, Nevitt MC, Browner W, Cauley J, Ensrud K, et al. Bone density at various sites for prediction of hip fractures. The study of osteoporotic fractures research group. Lancet. 1993;341(8837):72-5.

104. Black DM, Bauer DC, Vittinghoff E, Lui L-Y, Grauer A, Marin F, et al. Treatment-related changes in bone mineral density as a surrogate biomarker for fracture risk reduction: meta-regression analyses of individual patient data from multiple randomised controlled trials. Lancet Diabetes Endocrinol. 2020:8:672-82.

105. Sherrington C, Fairhall NJ, Wallbank GK, Tiedemann A, Michaleff ZA, Howard $\mathrm{K}$, et al. Exercise for preventing falls in older people living in the community. Cochrane Database Syst Rev. 2019;1:CD012424.

106. Howe TE, Rochester L, Neil F, Skelton DA, Ballinger C. Exercise for improving balance in older people. Cochrane Database Syst Rev. 2011:CD004963.

107. Australia TRACoGPaO. Osteoporosis prevention, diagnosis and management in postmenopausal women and men over 50 years of age. 2 nd ed. East Melbourne: RACGP; 2017.

108. Nikander R, Sievanen H, Heinonen A, Daly RM, Uusi-Rasi K, Kannus P. Targeted exercise against osteoporosis: a systematic review and metaanalysis for optimising bone strength throughout life. BMC Med. 2010;8:47.

\section{Publisher's Note}

Springer Nature remains neutral with regard to jurisdictional claims in published maps and institutional affiliations.

Ready to submit your research? Choose BMC and benefit from:

- fast, convenient online submission

- thorough peer review by experienced researchers in your field

- rapid publication on acceptance

- support for research data, including large and complex data types

- gold Open Access which fosters wider collaboration and increased citations

- maximum visibility for your research: over $100 \mathrm{M}$ website views per year

At $\mathrm{BMC}$, research is always in progress.

Learn more biomedcentral.com/submissions 